Supporting Information for:

\title{
Synthesis, Structure, and $\alpha$-Elimination Chemistry of Hafnocene Triarylstannyl Complexes
}

\author{
Nathan R. Neale and T. Don Tilley* \\ Contribution from the Department of Chemistry, University of California, Berkeley, \\ Berkeley, California 94720-1460 and the Center for New Directions in \\ Organic Synthesis, Berkeley, California 94720-1460
}

\section{EXPERIMENTAL DETAILS}

$\left[\mathbf{M e}_{2} \mathbf{C}\left(\mathbf{C}_{5} \mathbf{H}_{4}\right)_{2}\right] \mathbf{H f}\left(\mathbf{N M e}_{2}\right)_{2}(\mathbf{1}) .{ }^{13} \mathrm{C}\left\{{ }^{1} \mathrm{H}\right\}$ NMR: $\delta 25.0\left(\mathrm{~s},\left[\mathrm{Me}_{2} \mathrm{C}\left(\mathrm{C}_{5} \mathrm{H}_{4}\right)_{2}\right]\right), 36.9(\mathrm{~s}$, $\left.\left[\mathrm{Me}_{2} \mathrm{C}\left(\mathrm{C}_{5} \mathrm{H}_{4}\right)_{2}\right]\right), 48.6(\mathrm{~s}, \mathrm{NMe}), 102.1\left(\mathrm{~s},\left[\mathrm{Me}_{2} \mathrm{C}\left(C_{5} \mathrm{H}_{4}\right)_{2}\right]\right), 111.7\left(\mathrm{~s},\left[\mathrm{Me}_{2} \mathrm{C}\left(C_{5} \mathrm{H}_{4}\right)_{2}\right]\right), 128.0$ (s, [ $\left.\left.\mathrm{Me}_{2} \mathrm{C}\left(C_{5} \mathrm{H}_{4}\right)_{2}\right]\right) . \quad$ IR: $3121(\mathrm{v} \mathrm{w}), 3081(\mathrm{v} \mathrm{w}), 2814(\mathrm{~s}), 2761(\mathrm{~s}), 1454(\mathrm{~m}), 1423(\mathrm{w}), 1366(\mathrm{w}), 1239$ (m), $1134(\mathrm{~m}), 1078$ (w), 1057 (m), 1043 (m), 946 (s), $915(\mathrm{v} \mathrm{w}), 844$ (w), $832(\mathrm{w}), 794(\mathrm{~s}), 742(\mathrm{~m})$, 716 (w). mp $109-110^{\circ} \mathrm{C}$.

$\left[\mathbf{M e}_{2} \mathbf{C}\left(\mathbf{C}_{5} \mathbf{H}_{4}\right)_{2}\right] \mathbf{H f}\left(\mathbf{S n P h}_{3}\right) \mathbf{N M e}_{2}(\mathbf{2}) .{ }^{13} \mathrm{C}\left\{{ }^{1} \mathrm{H}\right\}$ NMR: $\delta 23.0\left(\mathrm{~s},\left[\mathrm{Me}_{2} \mathrm{C}\left(\mathrm{C}_{5} \mathrm{H}_{4}\right)_{2}\right]\right), 24.4(\mathrm{~s}$, $\left.\left[\mathrm{Me}_{2} \mathrm{C}\left(\mathrm{C}_{5} \mathrm{H}_{4}\right)_{2}\right]\right), 36.6\left(\mathrm{~s},\left[\mathrm{Me}_{2} \mathrm{C}\left(\mathrm{C}_{5} \mathrm{H}_{4}\right)_{2}\right]\right), 50.7$ (s, $\left.\mathrm{NMe} e_{2}\right), 99.9$ (s, [ $\left.\left.\mathrm{Me}_{2} \mathrm{C}\left(\mathrm{C}_{5} \mathrm{H}_{4}\right)_{2}\right]\right), 102.3$ (s, $\left.\left[\mathrm{Me}_{2} \mathrm{C}\left(C_{5} \mathrm{H}_{4}\right)_{2}\right]\right), 109.3\left(\mathrm{~s},\left[\mathrm{Me}_{2} \mathrm{C}\left(C_{5} \mathrm{H}_{4}\right)_{2}\right]\right), 111.4\left(\mathrm{~s},\left[\mathrm{Me}_{2} \mathrm{C}\left(C_{5} \mathrm{H}_{4}\right)_{2}\right]\right), 118.5\left(\mathrm{~s},\left[\mathrm{Me}_{2} \mathrm{C}\left(C_{5} \mathrm{H}_{4}\right)_{2}\right]\right), 127.7$ (s, $\left.p-C_{6} \mathrm{H}_{5}\right), 128.8\left(\mathrm{~s}, m-C_{6} \mathrm{H}_{5}\right), 138.5\left(\mathrm{~s}, o-C_{6} \mathrm{H}_{5},{ }^{2} J_{\mathrm{SnC}}=140 \mathrm{~Hz}\right), 151.7\left(\mathrm{~s}, i p s o-C_{6} \mathrm{H}_{5}\right)$. IR: $3087(\mathrm{w})$, $3056(\mathrm{~m}), 2819(\mathrm{~m}), 2772(\mathrm{w}), 1572(\mathrm{v} \mathrm{w}), 1425(\mathrm{~m}), 1263(\mathrm{~m}), 1240(\mathrm{w}), 1123(\mathrm{w}), 1066(\mathrm{~m}), 1041$ $(\mathrm{m}), 944(\mathrm{~m}), 806(\mathrm{~s}), 781(\mathrm{~m}), 728(\mathrm{~s}), 700(\mathrm{~s}), 649(\mathrm{v} \mathrm{w}), 449(\mathrm{~m}) . \mathrm{mp} 156-158{ }^{\circ} \mathrm{C}$.

CpCp*Hf(NMe $)$ Cl (5). $\mathrm{mp} 240{ }^{\circ} \mathrm{C}(\mathrm{dec})$.

CpCp*Hf(OMe)Cl (7). ${ }^{13} \mathrm{C}\left\{{ }^{1} \mathrm{H}\right\}$ NMR: $\delta 11.88\left(\mathrm{~s}, \mathrm{C}_{5} M e_{5}\right), 61.78$ (s, OMe), $112.5\left(\mathrm{~s}, C_{5} \mathrm{H}_{5}\right)$, $119.8\left(\mathrm{~s}, C_{5} \mathrm{Me}_{5}\right.$ ). IR: $3120(\mathrm{v} \mathrm{w}), 3100(\mathrm{v} \mathrm{w}), 3082(\mathrm{v} \mathrm{w}), 2807(\mathrm{~m}), 2728(\mathrm{v} \mathrm{w}), 1166(\mathrm{~m}), 1139(\mathrm{~s})$, $1069(\mathrm{w}), 905(\mathrm{w}), 882(\mathrm{w}), 815(\mathrm{~s}), 700(\mathrm{w}), 566(\mathrm{w}), 473(\mathrm{w}), 429(\mathrm{~s}), 407(\mathrm{~m}) . \mathrm{mp} 179-180{ }^{\circ} \mathrm{C}$.

CpCp*Hf(SnPh $\left.{ }_{3}\right) \mathbf{N M e}_{2} \bullet \mathbf{0 . 7 5}\left(\mathbf{C}_{4} \mathbf{H}_{10} \mathbf{O}\right)(\mathbf{8}) .{ }^{13} \mathrm{C}\left\{{ }^{1} \mathrm{H}\right\}$ NMR: $\delta 13.01\left(\mathrm{~s}, \mathrm{C}_{5} M e_{5}\right), 49.09(\mathrm{~s}$, $\left.\mathrm{N} M e_{2}\right), 109.5\left(\mathrm{~s}, C_{5} \mathrm{H}_{5}\right), 118.3\left(\mathrm{~s}, C_{5} \mathrm{Me}_{5}\right), 127.5\left(\mathrm{~s}, p-C_{6} \mathrm{H}_{5}\right), m-C_{6} \mathrm{H}_{5}$ coincident with the $C_{6} \mathrm{D}_{6}$ resonance, $139.0\left(\mathrm{~s},{ }^{2} J_{\mathrm{SnC}}=33.2 \mathrm{~Hz}, o-C_{6} \mathrm{H}_{5}\right), 152.5\left(\mathrm{~s}\right.$, ipso- $\left.C_{6} \mathrm{H}_{5}\right)$. IR: $3057(\mathrm{~m}), 2999(\mathrm{~m}), 2821(\mathrm{~m})$, $2775(\mathrm{~m}), 1574(\mathrm{w}), 1425(\mathrm{~m}), 1232(\mathrm{w}), 1115(\mathrm{w}), 1065(\mathrm{~m}), 1041(\mathrm{w}), 1028(\mathrm{~m}), 997(\mathrm{w}), 947(\mathrm{~s}), 849$ (w), 820 (s), 723 (s), 702 (s), 648 (w). mp $205-206{ }^{\circ} \mathrm{C}$ (dec).

$\mathbf{C p C p} * \mathbf{H f}\left(\mathbf{S n P h}_{3}\right) \mathbf{M e} \cdot \mathbf{0 . 5}\left(\mathbf{C}_{5} \mathbf{H}_{12}\right)(\mathbf{9}) .{ }^{13} \mathrm{C}\left\{{ }^{1} \mathrm{H}\right\}$ NMR: $\delta 12.77\left(\mathrm{~s},{ }^{3} J_{\mathrm{SnC}}=10.4 \mathrm{~Hz}, \mathrm{C}_{5} M e_{5}\right), 58.67$ $(\mathrm{s}, \mathrm{Hf}-\mathrm{Me}), 111.4\left(\mathrm{~s}, C_{5} \mathrm{H}_{5}\right), 119.1\left(\mathrm{~s}, C_{5} \mathrm{Me}_{5}\right), 127.6\left(\mathrm{~s}, p-C_{6} \mathrm{H}_{5}\right), 128.8\left(\mathrm{~s}, m-C_{6} \mathrm{H}_{5}\right), 138.7\left(\mathrm{~s},{ }^{2} J_{\mathrm{SnC}}=\right.$ $\left.34.4 \mathrm{~Hz}, o-C_{6} \mathrm{H}_{5}\right), 153.0$ (s, ipso- $C_{6} \mathrm{H}_{5}$ ). IR: 3057 (m), 3001 (br m), 1574 (w), 1558 (w), 1506 (w), 1489 (w), $1425(\mathrm{~m}), 1138(\mathrm{~m}), 1066(\mathrm{~m}), 997(\mathrm{w}), 820(\mathrm{~s}), 727(\mathrm{~s}), 702(\mathrm{~s}), 650(\mathrm{w}) . \mathrm{mp} 136-139{ }^{\circ} \mathrm{C}(\mathrm{dec})$.

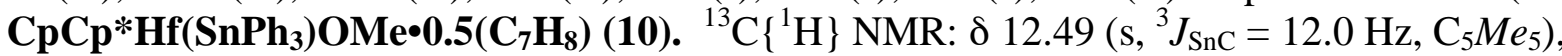
$62.16(\mathrm{~s}, \mathrm{OMe}), 109.6\left(\mathrm{~s}, C_{5} \mathrm{H}_{5}\right), 118.1\left(\mathrm{~s}, C_{5} \mathrm{Me}_{5}\right), 127.6\left(\mathrm{~s}, p-C_{6} \mathrm{H}_{5}\right), 128.7\left(\mathrm{~s}, m-C_{6} \mathrm{H}_{5}\right), 138.8\left(\mathrm{~s},{ }^{2} J_{\mathrm{SnC}}=\right.$ $\left.34.6 \mathrm{~Hz}, o-C_{6} \mathrm{H}_{5}\right), 152.3\left(\mathrm{~s}\right.$, ipso- $\left.C_{6} \mathrm{H}_{5}\right)$. IR: $3054(\mathrm{~m}), 2811(\mathrm{~m}), 2726(\mathrm{~m}), 1573(\mathrm{~m}), 1425(\mathrm{~m}), 1300$ (v w), 1139 (s), $1118(\mathrm{~s}), 1065(\mathrm{~m}), 1011(\mathrm{~m}), 814(\mathrm{~s}), 729(\mathrm{~s}), 704(\mathrm{~s}) . \mathrm{mp} 178-179{ }^{\circ} \mathrm{C}$.

CpCp*Hf(Ph)Cl (12). ${ }^{13} \mathrm{C}\left\{{ }^{1} \mathrm{H}\right\}$ NMR $\left(\mathrm{C}_{7} \mathrm{D}_{8},-70{ }^{\circ} \mathrm{C}\right): \delta 12.28\left(\mathrm{~s}, \mathrm{C}_{5} M e_{5}\right), 113.7\left(\mathrm{~s}, C_{5} \mathrm{H}_{5}\right)$, $120.4\left(\mathrm{~s}, C_{5} \mathrm{Me}_{5}\right), 125.2\left(\mathrm{~s}, m-\mathrm{C}_{6} H_{5}\right), 127.8\left(\mathrm{~s}, m-\mathrm{C}_{6} H_{5}\right), p-\mathrm{C}_{6} H_{5}$ was coincident with an aryl $C_{7} \mathrm{D}_{8}$ resonance, $139.7\left(\mathrm{~s}, o-\mathrm{C}_{6} H_{5}\right), 139.9\left(\mathrm{~s}, o-\mathrm{C}_{6} H_{5}\right), 196.1\left(\mathrm{~s}, i p s o-\mathrm{C}_{6} H_{5}\right)$. IR: $3126(\mathrm{v} \mathrm{w}), 3112(\mathrm{v} \mathrm{w}), 3051$ (w), $3037(\mathrm{w}), 1489(\mathrm{w}), 1458(\mathrm{~m}), 1413(\mathrm{w}), 1308(\mathrm{v} \mathrm{w}), 1308(\mathrm{v} \mathrm{w}), 1233(\mathrm{v} \mathrm{w}), 1091(\mathrm{w}), 1060(\mathrm{w})$, $814(\mathrm{~s}), 724(\mathrm{~m}), 704(\mathrm{~m}) . \mathrm{mp} 165-167{ }^{\circ} \mathrm{C}$.

CpCp*Hf(H)Ph (13). ${ }^{13} \mathrm{C}\left\{{ }^{1} \mathrm{H}\right\}$ NMR: $\delta 12.48\left(\mathrm{~s}, \mathrm{C}_{5} M e_{5}\right), 110.1\left(\mathrm{~s}, C_{5} \mathrm{H}_{5}\right), 118.1\left(\mathrm{~s}, C_{5} \mathrm{Me}_{5}\right)$, $125.8\left(\mathrm{~s}, p-C_{6} \mathrm{H}_{5}\right), 127.5\left(\mathrm{~s}, m-C_{6} \mathrm{H}_{5}\right), o-C_{6} \mathrm{H}_{5}$ not observed, 201.2 (s, ipso- $\left.C_{6} \mathrm{H}_{5}\right)$. IR: 3111 (v w), 3042 
(m), $3015(\mathrm{w}), 2726(\mathrm{w}), 1600\left(\mathrm{~s}, \mathrm{v}_{\mathrm{HfH}}\right), 1555(\mathrm{w}), 1489(\mathrm{~m}), 1413(\mathrm{~m}), 1059(\mathrm{~m}), 804(\mathrm{~s}), 749(\mathrm{~m}), 723$ (s), 703 (s), $644(\mathrm{v} \mathrm{w}) \cdot \operatorname{mp} 90-94{ }^{\circ} \mathrm{C}$.

CpCp*Hf(SnPh $\left.{ }_{3}\right) \mathbf{O}^{\mathbf{t}} \mathbf{B u} .{ }^{1} \mathrm{H}$ NMR: $\delta 1.04\left(\mathrm{~s}, 9 \mathrm{H}, \mathrm{O}^{t} B u\right), 1.83\left(\mathrm{~s}, 15 \mathrm{H}, \mathrm{C}_{5} M e_{5}\right), 5.94\left(\mathrm{~s},{ }^{3} \mathrm{~J}_{\mathrm{SnH}}=\right.$ $\left.6.4 \mathrm{~Hz}, 5 \mathrm{H}, \mathrm{C}_{5} H_{5}\right), 7.90-7.93\left(\mathrm{~m}, 6 \mathrm{H}, o-\mathrm{C}_{6} H_{5}\right)$. The $m$ - and $p-\mathrm{C}_{6} H_{5}$ resonances were coincident with other phenyl resonances and could not be definitively identified.

CpCp*Hf(SnPh $) \mathbf{N p}(\mathbf{1 4}) .{ }^{13} \mathrm{C}\left\{{ }^{1} \mathrm{H}\right\} \mathrm{NMR}: \delta 12.24\left(\mathrm{~s},{ }^{3} \mathrm{~J}_{\mathrm{SnC}}=11.5 \mathrm{~Hz}, \mathrm{C}_{5} M e_{5}\right), 35.80(\mathrm{~s}$, $\mathrm{CH}_{2} \mathrm{CMe}_{3}$ ), 40.95 (s, $\mathrm{CH}_{2} \mathrm{CMe}_{3}$ ), 108.5 (s, $C_{5} \mathrm{H}_{5}$ ), 118.1 (s, $C_{5} \mathrm{Me}_{5}$ ), 124.8 (weak m, $\mathrm{CH}_{2} \mathrm{CMe}_{3}$ ), 127.7 $\left(\mathrm{s}, p-C_{6} \mathrm{H}_{5}\right), 128.7\left(\mathrm{~s}, m-C_{6} \mathrm{H}_{5}\right), 138.7\left(\mathrm{~s},{ }^{2} J_{\mathrm{SnC}}=33.4 \mathrm{~Hz}, o-C_{6} \mathrm{H}_{5}\right), 152.5\left(\mathrm{~s}\right.$, ipso- $\left.C_{6} \mathrm{H}_{5}\right)$. IR $(\mathrm{KBr}): 3053$ (m), 3000 (m), $2974(\mathrm{~m}), 2951$ (s), 2905 (m), 2862 (m), 1573 (v w), 1475 (m), 1458 (m), 1425 (s), 1379 (m), $1228(\mathrm{w}), 1064(\mathrm{~m}), 1020(\mathrm{~m}), 997(\mathrm{v} \mathrm{w}), 817(\mathrm{~s}), 724(\mathrm{~s}), 701(\mathrm{~s}), 461(\mathrm{~m})$.

$\mathbf{H S n}\left[p \text {-(OMe) } \mathbf{C}_{6} \mathbf{H}_{4}\right]_{3}(\mathbf{1 5}) .{ }^{1} \mathrm{H}$ NMR: $\delta 3.29\left(\mathrm{~s}, 9 \mathrm{H}, p-(\mathrm{OMe}) \mathrm{C}_{6} \mathrm{H}_{4}\right), 6.86(\mathrm{~m}, 6 \mathrm{H}, m-$ $\left.(\mathrm{OMe}) \mathrm{C}_{6} H_{4}\right), 6.94\left(\mathrm{~s},{ }^{1} J_{117 / 119 \mathrm{SnH}}=1829,1924 \mathrm{~Hz}, 1 \mathrm{H}, \mathrm{Sn}-H\right), 7.47-7.58\left(\mathrm{~m}, 6 \mathrm{H}, o-(\mathrm{OMe}) \mathrm{C}_{6} H_{4}\right)$. ${ }^{13} \mathrm{C}\left\{{ }^{1} \mathrm{H}\right\}$ NMR: $\delta 54.9\left(\mathrm{~s},(\mathrm{OMe}) \mathrm{C}_{6} \mathrm{H}_{4}\right), 115.4\left(\mathrm{~s},{ }^{3} J_{\mathrm{SnC}}=57.7 \mathrm{~Hz}, m-(\mathrm{OMe}) C_{6} \mathrm{H}_{4}\right), 128.3(\mathrm{~s}, p-$ $\left.(\mathrm{OMe}) C_{6} \mathrm{H}_{4}\right), 139.2\left(\mathrm{~s},{ }^{2} J_{\mathrm{SnC}}=46.4 \mathrm{~Hz}, o-(\mathrm{OMe}) C_{6} \mathrm{H}_{4}\right), 161.5\left(\mathrm{~s},{ }^{1} J_{\mathrm{SnC}}=12.6 \mathrm{~Hz}\right.$, ipso- $\left.(\mathrm{OMe}) C_{6} \mathrm{H}_{4}\right) . \mathrm{IR}$ : $3053(\mathrm{w}), 3042(\mathrm{w}), 3011(\mathrm{w}), 2518(\mathrm{v} \mathrm{w}), 2045(\mathrm{v} \mathrm{w}), 2031(\mathrm{v} \mathrm{w}), 1895\left(\mathrm{~m}, \mathrm{v}_{\mathrm{SnH}}\right), 1584(\mathrm{~s}), 1561(\mathrm{~m})$, 1494 (s), 1439 (m), 1393 (w), 1310 (w), 1277 (s), 1244 (s), 1180 (s), 1118 (w), 1074 (s), 1028 (s), 833 (m), $817(\mathrm{~s}), 788(\mathrm{~m}) . \mathrm{mp} 104-107^{\circ} \mathrm{C}$.

$\left.\mathbf{H S n}\left(\boldsymbol{p}-\mathbf{F C}_{6} \mathbf{H}_{4}\right)_{3} \mathbf{( 1 6}\right)$. An $\mathrm{Et}_{2} \mathrm{O}(20 \mathrm{~mL})$ solution of $\mathrm{ClSn}\left(p-\mathrm{FC}_{6} \mathrm{H}_{4}\right)_{3}(0.506 \mathrm{~g}, 1.15 \mathrm{mmol})$ was added to a suspension of $\mathrm{LAH}(0.019 \mathrm{~g}, 0.50 \mathrm{mmol}, 0.43$ equiv $)$ in $\mathrm{Et}_{2} \mathrm{O}(60 \mathrm{~mL})$ at $0{ }^{\circ} \mathrm{C}$, and the resulting reaction mixture was gradually warmed to room temperature with stirring for $1.5 \mathrm{~h}$ in the dark. The cloudy mixture was quenched with distilled water $\left(1 \mathrm{~mL}\right.$, deoxygenated by sparging with $\mathrm{N}_{2}$ for $2 \mathrm{~h}$; CAUTION: causes vigorous $\mathrm{H}_{2}$ gas evolution), and was then washed with more distilled, deoxygenated water $(50 \mathrm{~mL})$. The organic layer was washed with two additional portions $(40 \mathrm{~mL})$ of distilled, deoxygenated water, and then the ether solution was dried over ca. $5 \mathrm{~g}$ of $\mathrm{CaCl}_{2}: \mathrm{MgSO}_{4}(1: 1$, w/w $)$ for 2 $\mathrm{h}$. After filtration, the beige solution was concentrated to dryness, leaving the product as a pale-yellow oil that solidified into a crystalline solid over a few days; $89 \%$ yield $(0.413 \mathrm{~g}, 1.02 \mathrm{mmol}) .{ }^{1} \mathrm{H} \mathrm{NMR}: \delta$ $6.69\left(\mathrm{~s},{ }^{1} J_{117 / 119 \mathrm{SnH}}=1911,2000 \mathrm{~Hz}, 1 \mathrm{H}, \mathrm{Sn}-H\right), 6.84\left(\mathrm{~m}, 6 \mathrm{H}, m-\mathrm{FC}_{6} H_{4}\right), 7.15\left(\mathrm{~m}, 6 \mathrm{H}, o-\mathrm{FC}_{6} H_{4}\right)$. ${ }^{13} \mathrm{C}\left\{{ }^{1} \mathrm{H}\right\}$ NMR: $\delta 116.7\left(\mathrm{~s},{ }^{2} J_{\mathrm{FC}}=19.5 \mathrm{~Hz},{ }^{3} J_{117 / 119 \mathrm{SnC}}=56.3,58.9 \mathrm{~Hz}, m-\mathrm{F} C_{6} \mathrm{H}_{4}\right), 131.0\left(\mathrm{~s},{ }^{4} J_{\mathrm{FC}}=4.5\right.$ $\mathrm{Hz}$, ipso- $\left.\mathrm{FC}_{6} \mathrm{H}_{4}\right), 139.5\left(\mathrm{~s},{ }^{3} J_{\mathrm{FC}}=7.1 \mathrm{~Hz},{ }^{2} J_{117 / 119 \mathrm{SnC}}=45.1,47.1 \mathrm{~Hz}, o-\mathrm{FC}_{6} \mathrm{H}_{4}\right), 161.5\left(\mathrm{~s},{ }^{1} J_{\mathrm{FC}}=248 \mathrm{~Hz}\right.$, $\left.{ }^{4} J_{\mathrm{SnC}}=13.4 \mathrm{~Hz}, p-\mathrm{FC}_{6} \mathrm{H}_{4}\right) .{ }^{19} \mathrm{~F}\left\{{ }^{1} \mathrm{H}\right\} \mathrm{NMR}: \delta-110.8\left(\mathrm{~s}, F \mathrm{C}_{6} \mathrm{H}_{4}\right) .{ }^{119} \mathrm{Sn}\left\{{ }^{1} \mathrm{H}\right\} \mathrm{NMR}: \delta-156.6$ (s). Anal. Calcd. for $\mathrm{C}_{18} \mathrm{H}_{13} \mathrm{~F}_{3} \mathrm{Sn}$ : C, 53.38; H, 3.24. Found: C, 53.03; H, 3.56. IR: 3165 (v w), 3086 (v w), 3058 (w), $3022(\mathrm{w}), 1874\left(\mathrm{~s}, \mathrm{v}_{\mathrm{SnH}}\right), 1642(\mathrm{v} \mathrm{w}), 1582(\mathrm{~s}), 1492(\mathrm{~s}), 1445(\mathrm{~m}), 1328(\mathrm{v} \mathrm{w}), 1306(\mathrm{~m}), 1226(\mathrm{~s})$, 1163 (s), 1067 (m), 817 (s), 703 (v w). $\mathrm{mp} 37-39^{\circ} \mathrm{C}$.

$\mathbf{H S n}\left[\boldsymbol{p}-\left(\mathbf{C F}_{\mathbf{3}}\right) \mathbf{C}_{\mathbf{6}} \mathbf{H}_{4}\right]_{3} \mathbf{( 1 7 )}$. An $\mathrm{Et}_{2} \mathrm{O}(20 \mathrm{~mL})$ solution of $\mathrm{ClSn}\left[p-\left(\mathrm{CF}_{3}\right) \mathrm{C}_{6} \mathrm{H}_{4}\right]_{3}(1.80 \mathrm{~g}, 3.05$ mmol) was added to a suspension of $\mathrm{LAH}(0.050 \mathrm{~g}, 1.32 \mathrm{mmol}, 0.43 \mathrm{equiv})$ in $\mathrm{Et}_{2} \mathrm{O}(60 \mathrm{~mL})$ at $0{ }^{\circ} \mathrm{C}$, and the resulting reaction mixture was gradually warmed to room temperature with stirring in the dark for $1.5 \mathrm{~h}$. The cloudy mixture was quenched with distilled water $(1 \mathrm{~mL}$, deoxygenated by sparging with $\mathrm{N}_{2}$ for $2 \mathrm{~h}$; CAUTION: causes vigorous $\mathrm{H}_{2}$ gas evolution), and was then washed with more distilled, deoxygenated water $(60 \mathrm{~mL})$. The organic layer was washed with two additional portions $(60 \mathrm{~mL})$ of distilled, deoxygenated water, and then the ether solution was dried over ca. $5 \mathrm{~g}$ of $\mathrm{CaCl}_{2}: \mathrm{MgSO}_{4}(1: 1$, $\mathrm{w} / \mathrm{w})$ for $2 \mathrm{~h}$. After filtration, the colorless solution was concentrated to dryness, leaving the impure product as an opaque oil. The impure product was crystallized from pentane $(10 \mathrm{~mL})$ at $-30{ }^{\circ} \mathrm{C}$ to give the product as a colorless crystalline solid in $55 \%$ yield $(0.541 \mathrm{~g}, 0.975 \mathrm{mmol}) .{ }^{1} \mathrm{H} \mathrm{NMR:} \delta 6.68(\mathrm{~s}$, $\left.{ }^{1} J_{117 / 119 \mathrm{SnH}}=1983,2076 \mathrm{~Hz}, 1 \mathrm{H}, \mathrm{Sn}-H\right), 7.15\left(\mathrm{~m}, 6 \mathrm{H}, m-\left(\mathrm{CF}_{3}\right) \mathrm{C}_{6} H_{4}\right), 7.36\left(\mathrm{~m}, 6 \mathrm{H}, o-\left(\mathrm{CF}_{3}\right) \mathrm{C}_{6} H_{4}\right)$. ${ }^{13} \mathrm{C}\left\{{ }^{1} \mathrm{H}\right\}$ NMR: $\delta 125.1\left(\mathrm{q},{ }^{1} J_{\mathrm{FC}}=272 \mathrm{~Hz},\left(C_{3}\right) \mathrm{C}_{6} \mathrm{H}_{4}\right), 125.8\left(\mathrm{q},{ }^{3} J_{\mathrm{FC}}=3.8 \mathrm{~Hz}, m-\left(\mathrm{CF}_{3}\right) C_{6} \mathrm{H}_{4}\right), 132.3(\mathrm{q}$, $\left.{ }^{2} J_{\mathrm{FC}}=32.3 \mathrm{~Hz}, p-\left(\mathrm{CF}_{3}\right) C_{6} \mathrm{H}_{4}\right), 138.0\left(\mathrm{~s}, o-\left(\mathrm{CF}_{3}\right) C_{6} \mathrm{H}_{4}\right), 141.2\left(\mathrm{q},{ }^{5} J_{\mathrm{FC}}=1.2 \mathrm{~Hz}\right.$, ipso- $\left.\left(\mathrm{CF}_{3}\right) C_{6} \mathrm{H}_{4}\right)$. 
${ }^{19} \mathrm{~F}\left\{{ }^{1} \mathrm{H}\right\}$ NMR: $\delta-62.76\left(\mathrm{~s},\left(\mathrm{CF}_{3}\right) \mathrm{C}_{6} \mathrm{H}_{4}\right) .{ }^{119} \mathrm{Sn}\left\{{ }^{1} \mathrm{H}\right\} \mathrm{NMR}: \delta-166.2(\mathrm{~m})$. Anal. Calcd. for $\mathrm{C}_{21} \mathrm{H}_{13} \mathrm{~F}_{9} \mathrm{Sn}$ : C, 45.44; H, 2.36. Found: C, 45.15; H, 2.24. IR: 3062 (v w), 3033 (v w), 1879 (m, $\left.v_{\mathrm{SnH}}\right), 1601$ (m), 1458 (m), 1392 (m), 1328 (br, s), 1235 (v w), 1160 (br, s), 1100 (s), 1086 (s), 1051 (s), 955 (v w), 824 (s), $726(\mathrm{v} \mathrm{w}), 682(\mathrm{~m}), 599(\mathrm{~m}), 562(\mathrm{br}, \mathrm{s}) . \mathrm{mp} 85-88^{\circ} \mathrm{C}$.

CpCp*Hf $\left\{\mathbf{S n}\left[\boldsymbol{p} \text {-(OMe) } \mathbf{C}_{6} \mathbf{H}_{4}\right]_{3}\right\} \mathbf{C l}(\mathbf{1 8}) .{ }^{13} \mathrm{C}\left\{{ }^{1} \mathrm{H}\right\}$ NMR: $\delta 13.1\left(\mathrm{~s}, \mathrm{C}_{5} M e_{5}\right), 54.9\left(\mathrm{~s},(\mathrm{OMe}) \mathrm{C}_{6} \mathrm{H}_{4}\right)$ $112.1\left(\mathrm{~s}, C_{5} \mathrm{H}_{5}\right), 115.0\left(\mathrm{~s},{ }^{3} J_{\mathrm{SnC}}=33.7 \mathrm{~Hz}, m-(\mathrm{OMe}) C_{6} \mathrm{H}_{4}\right), 120.9\left(\mathrm{~s}, C_{5} \mathrm{Me}_{5}\right), 139.7\left(\mathrm{~s},{ }^{2} J_{\mathrm{SnC}}=37.4 \mathrm{~Hz}\right.$, $\left.o-(\mathrm{OMe}) C_{6} \mathrm{H}_{4}\right), 142.3\left(\mathrm{~s}, p-(\mathrm{OMe}) C_{6} \mathrm{H}_{4}\right), 160.2$ (s, ipso-(OMe) $\left.C_{6} \mathrm{H}_{4}\right)$. IR: $3040(\mathrm{w}), 3007(\mathrm{~m}), 1582(\mathrm{~s})$, $1561(\mathrm{~m}), 1490$ (s), 1439 (m), 1307 (v w), 1272 (s), 1238 (s), 1179 (s), 1065 (m), 1027 (m), 819 (s), 786 (m), 729 (v w). mp $166-167^{\circ} \mathrm{C}$.

CpCp*Hf[Sn(p-FC $\left.\left.6 \mathbf{H}_{4}\right)_{3}\right] \mathrm{Cl}(\mathbf{1 9}) .{ }^{13} \mathrm{C}\left\{{ }^{1} \mathrm{H}\right\} \mathrm{NMR}: \delta 13.0\left(\mathrm{~s},{ }^{3} J_{\mathrm{SnC}}=11.0 \mathrm{~Hz}, \mathrm{C}_{5} M e_{5}\right), 112.1(\mathrm{~s}$, $\left.C_{5} \mathrm{H}_{5}\right), 116.0\left(\mathrm{~d},{ }^{2} J_{\mathrm{FC}}=18.6 \mathrm{~Hz},{ }^{3} J_{\mathrm{SnC}}=33.0 \mathrm{~Hz}, m-\mathrm{FC}_{6} \mathrm{H}_{4}\right), 121.2\left(\mathrm{~s}, C_{5} \mathrm{Me}_{5}\right), 139.9\left(\mathrm{~d},{ }^{3} J_{\mathrm{FC}}=6.1 \mathrm{~Hz}\right.$, $\left.{ }^{2} J_{\mathrm{SnC}}=38.6 \mathrm{~Hz}, o-\mathrm{FC}_{6} \mathrm{H}_{4}\right), 146.2\left(\mathrm{~d},{ }^{4} J_{\mathrm{FC}}=3.8 \mathrm{~Hz}\right.$, ipso-FC $\left.C_{6} \mathrm{H}_{4}\right), 163.9\left(\mathrm{~d},{ }^{1} J_{\mathrm{FC}}=247 \mathrm{~Hz}, p-\mathrm{FC}_{6} \mathrm{H}_{4}\right)$.

${ }^{19} \mathrm{~F}\left\{{ }^{1} \mathrm{H}\right\}$ NMR: $\delta-114.3\left(\mathrm{~m}, F_{6} \mathrm{H}_{4}\right)$. IR: $3150(\mathrm{v} \mathrm{w}), 3112(\mathrm{w}), 3074(\mathrm{w}), 3053(\mathrm{w}), 3018(\mathrm{w}), 1652(\mathrm{v}$ w), $1576(\mathrm{~s}), 1486(\mathrm{~s}), 1457(\mathrm{~m}), 1306(\mathrm{v} \mathrm{w}), 1220(\mathrm{~s}), 1160(\mathrm{~s}), 1082(\mathrm{w}), 1052(\mathrm{~m}), 942(\mathrm{v} \mathrm{w}), 816$ (s), 722 (v w). $\operatorname{mp} 184-186^{\circ} \mathrm{C}$.

CpCp*Hf $\left\{\operatorname{Sn}\left[p-\left(\mathbf{C F}_{3}\right) \mathbf{C}_{6} \mathbf{H}_{4}\right]_{3}\right\} \mathrm{Cl}(\mathbf{2 0}) .{ }^{13} \mathrm{C}\left\{{ }^{1} \mathrm{H}\right\}$ NMR: $\delta 13.0\left(\mathrm{~s},{ }^{3} J_{\mathrm{SnC}}=11.5 \mathrm{~Hz}, \mathrm{C}_{5} M e_{5}\right), 112.1$ $\left(\mathrm{s}, C_{5} \mathrm{H}_{5}\right), 121.2\left(\mathrm{~s}, C_{5} \mathrm{Me}_{5}\right), 125.3\left(\mathrm{q},{ }^{3} J_{\mathrm{FC}}=3.8 \mathrm{~Hz}, m-\left(\mathrm{CF}_{3}\right) C_{6} \mathrm{H}_{4}\right), 125.7\left(\mathrm{q},\left(C \mathrm{~F}_{3}\right) \mathrm{C}_{6} \mathrm{H}_{4},{ }^{1} J_{\mathrm{FC}}=272 \mathrm{~Hz}\right)$, $130.4\left(\mathrm{q}, p-\left(\mathrm{CF}_{3}\right) C_{6} \mathrm{H}_{4},{ }^{2} J_{\mathrm{FC}}=31.8 \mathrm{~Hz}\right), 138.5\left(\mathrm{~s},{ }^{2} J_{\mathrm{SnC}}=34.6 \mathrm{~Hz}, o-\left(\mathrm{CF}_{3}\right) C_{6} \mathrm{H}_{4}\right), 156.2\left(\mathrm{q},{ }^{5} J_{\mathrm{FC}}=1.2 \mathrm{~Hz}\right.$, ipso- $\left.\left(\mathrm{CF}_{3}\right) C_{6} \mathrm{H}_{4}\right) .{ }^{19} \mathrm{~F}\left\{{ }^{1} \mathrm{H}\right\} \mathrm{NMR}: \delta-62.20\left(\mathrm{~s},\left(\mathrm{CF}_{3}\right) C_{6} \mathrm{H}_{4}\right)$. IR: $3188(\mathrm{v} \mathrm{w}), 3113(\mathrm{v} \mathrm{w}), 3073(\mathrm{v} \mathrm{w})$, 3055 (v w), 3023 (w), 1596 (m), 1488 (w), 1459 (s), 1321 (s), 1286 (m), 1162 (s), 1119 (s), 1097 (s), 1080 (s), 1043 (s), 954 (w), 822 (s), 724 (w), 676 (m), 597 (m). mp 179 - $182{ }^{\circ} \mathrm{C}$.

X-ray Structure Determinations. X-ray diffraction measurements were made on a Siemens SMART diffractometer with a CCD area detector, using graphite-monochromated MoKa radiation. The crystal was mounted on a glass fiber using Paratone $\mathrm{N}$ hydrocarbon oil. A hemisphere of data was collected using $\omega$ scans of $0.3^{\circ}$. Cell constants and an orientation matrix for data collection were obtained from a least-squares refinement using the measured positions of reflections in the range $3^{\circ} \leq 2 \theta$ $\leq 52.2^{\circ}$. The frame data were integrated using the program SAINT (SAX Area-Detector Integration Program; V4.024; Siemens Industrial Automation, Inc.: Madison, WI, 1995). An empirical absorption correction based on measurements of multiply redundant data was performed using the program SADABS. Equivalent reflections were merged. The data were corrected for Lorentz and polarization effects. A secondary extinction correction was applied where appropriate. The structures were solved using the teXsan crystallographic software package of the Molecular Structure Corporation, using direct methods, and expanded with Fourier techniques. All non-hydrogen atoms were refined anisotripically unless otherwise noted, and the hydrogen atoms were included in calculated positions but not refined. The function minimized in the full-matrix least-squares refinement was $\Sigma \omega(|\mathrm{Fo}|-|\mathrm{Fc}|)^{2}$. The weighting scheme was based on counting statistics and included a p-factor to downweight the intense reflections. Crystallographic data are summarized in the Supplementary Information.

For 2. Crystals were grown from a concentrated $\mathrm{Et}_{2} \mathrm{O}$ solution at $-30^{\circ} \mathrm{C}$.

For 4. Crystals were grown from a concentrated $\mathrm{Et}_{2} \mathrm{O}$ solution at $-30{ }^{\circ} \mathrm{C}$.

For 8•0.75( $\left.\mathbf{E t}_{2} \mathrm{O}\right)$. Crystals were grown from a concentrated $\mathrm{Et}_{2} \mathrm{O}$ solution at $-30^{\circ} \mathrm{C}$. One and one-half molecules of $\mathrm{Et}_{2} \mathrm{O}$ were found in the unit cell (note that two molecules of $\mathbf{8}$ were found in the unit cell). For the one full-occupancy $\mathrm{Et}_{2} \mathrm{O}$ molecule, the atoms were refined isotropically. For the halfoccupancy $\mathrm{Et}_{2} \mathrm{O}$ molecule, an inversion center was centered in between the $\mathrm{C}-\mathrm{O}$ bond, and thus this was 
modeled as one $7 / 8^{\text {th }}$-occupancy oxygen, one full-occupancy carbon, and one half-occupancy carbon, which were refined isotropically. The other atoms of the $\mathrm{Et}_{2} \mathrm{O}$ molecule were generated using a full symmetry expansion.

For $9 \bullet \mathbf{0 . 5}\left(\mathbf{C}_{\mathbf{5}} \mathbf{H}_{12}\right)$. Crystals were grown from a concentrated pentane solution at $-30^{\circ} \mathrm{C}$. A molecule of pentane was found in the unit cell with the central carbon atom on an inversion center. This molecule was modeled as a half-occupancy carbon (on the inversion center) and two full-occupancy carbons which were refined isotropically. The other atoms of the pentane molecule were generated using a full symmetry expansion.

For $\mathbf{1 0} \boldsymbol{0 . 5}\left(\mathbf{C}_{\mathbf{7}} \mathbf{H}_{\mathbf{8}}\right)$. Crystals were grown from a concentrated toluene solution at $-30^{\circ} \mathrm{C}$. The unit cell contained a half molecule of toluene that was located about an inversion center. This molecule was modeled as two full-occupancy carbons and three half-occupancy carbons, which were refined isotropically. The other atoms of the toluene molecule were generated using a full symmetry expansion.

Table 1. Crystal Data for $\left[\mathrm{Me}_{2} \mathrm{C}\left(\mathrm{C}_{5} \mathrm{H}_{4}\right)_{2}\right] \mathrm{Hf}\left(\mathrm{SnPh}_{3}\right) \mathrm{NMe}_{2}(2)$

Empirical Formula

Formula Weight

Crystal Color, Habit

Crystal Dimensions

Crystal System

Lattice Type

No. of Reflections Used for Unit

Cell Determination (2 $\theta$ range)

Lattice Parameters
Space Group

$\mathrm{Z}$ value

$\mathrm{D}_{\text {calc }}$

$\mathrm{F}_{000}$

$\mu(\operatorname{MoK} \alpha)$
$\mathrm{C}_{33} \mathrm{H}_{35} \mathrm{NSnHf}$

742.83

yellow, trapezoid

$0.25 \times 0.13 \times 0.10 \mathrm{~mm}$

monoclinic

C-centered

$$
\begin{aligned}
& 5383\left(3.0-52.2^{\mathrm{O}}\right) \\
& \mathrm{a}=10.9891(2) \AA \\
& \mathrm{b}=17.2808(2) \AA \\
& \mathrm{c}=15.5453(3) \AA \\
& \beta=106.022(1)^{\mathrm{O}} \\
& \mathrm{V}=2837.39(8) \AA^{3} \\
& \mathrm{Cc}(\# 9) \\
& 4 \\
& 1.739 \mathrm{~g} / \mathrm{cm}^{3} \\
& 1448.00 \\
& 45.51 \mathrm{~cm}^{-1}
\end{aligned}
$$

Table 2. Intensity Measurements for $\left[\mathrm{Me}_{2} \mathrm{C}\left(\mathrm{C}_{5} \mathrm{H}_{4}\right)_{2}\right] \mathrm{Hf}\left(\mathrm{SnPh}_{3}\right) \mathrm{NMe}_{2}$ (2)

Diffractometer

Radiation

Crystal to Detector Distance

Temperature

Scan Type

Scan Rate

$2 \theta_{\max }$
SMART

$\operatorname{MoK} \alpha(1=0.71069 \AA)$

graphite monochromated

$60.0 \mathrm{~mm}$

$-110.0^{\circ} \mathrm{C}$

$\omega\left(0.3^{\circ}\right.$ per frame $)$

10 seconds per frame

$52.2^{0}$ 
No. of Reflections Measured

Corrections
Total: 13781

Unique: $5239\left(\mathrm{R}_{\text {int }}=0.038\right)$

Lorentz-polarization

Absorption $(\mathrm{Tmax}=0.56229, \mathrm{Tmin}=0.40907)$

Table 3. Structure Solution and Refinement for $\left[\mathrm{Me}_{2} \mathrm{C}\left(\mathrm{C}_{5} \mathrm{H}_{4}\right)_{2}\right] \mathrm{Hf}\left(\mathrm{SnPh}_{3}\right) \mathrm{NMe}_{2}(2)$

Structure Solution

Refinement

Function Minimized

Least Squares Weights

p-factor

Anomalous Dispersion

No. Observations (I>3.00 $\sigma(\mathrm{I})$ )

No. Variables

Residuals: R; Rw; Rall

Goodness of Fit Indicator

Max Shift/Error in Final Cycle

Maximum peak in Final Diff. Map

Minimum peak in Final Diff. Map
Direct Methods (SIR92)

Full-matrix least-squares

$\Sigma \omega(|\mathrm{Fo}|-|\mathrm{Fc}|)^{2}$

$1 / \mathrm{\sigma}^{2}(\mathrm{Fo})=4 \mathrm{Fo}^{2} / \mathrm{\sigma}^{2}\left(\mathrm{Fo}^{2}\right)$

0.030

All non-hydrogen atoms

2300

318

$0.025 ; 0.029 ; 0.029$

1.04

0.00

$0.93 \mathrm{e}^{-} / \AA^{3}$

$-1.44 \mathrm{e}^{-} / \AA^{3}$

Table 4. Atomic coordinates and $\mathrm{B}_{\text {iso }} / \mathrm{B}_{\text {eq }}$ for $\left[\mathrm{Me}_{2} \mathrm{C}\left(\mathrm{C}_{5} \mathrm{H}_{4}\right)_{2}\right] \mathrm{Hf}\left(\mathrm{SnPh}_{3}\right) \mathrm{NMe}_{2}(2)$

$\begin{array}{llcll}\text { atom } & \mathrm{x} & \mathrm{y} & \mathrm{z} & \mathrm{B} \text { eq } \\ \mathrm{Hf}(1) & 0.1382 & 0.04950(2) & 0.7093 & 1.824(8) \\ \mathrm{Sn}(1) & 0.22386(7) & 0.20457(3) & 0.77823(5) & 1.98(1) \\ \mathrm{N}(1) & 0.2871(7) & -0.0051(4) & 0.7983(5) & 2.1(2) \\ \mathrm{C}(1) & 0.0971(10) & 0.0966(6) & 0.5502(6) & 2.5(2) \\ \mathrm{C}(2) & 0.2327(9) & 0.1030(6) & 0.5834(7) & 2.5(2) \\ \mathrm{C}(3) & 0.279(1) & 0.0261(9) & 0.6031(8) & 3.3(3) \\ \mathrm{C}(4) & 0.1741(9) & -0.0260(6) & 0.5833(7) & 2.2(2) \\ \mathrm{C}(5) & 0.0607(9) & 0.0180(7) & 0.5493(7) & 2.5(2) \\ \mathrm{C}(6) & -0.0740(9) & -0.0092(7) & 0.5441(7) & 2.2(2) \\ \mathrm{C}(7) & -0.089(1) & -0.0972(7) & 0.5218(8) & 3.1(2) \\ \mathrm{C}(8) & -0.1714(10) & 0.0355(7) & 0.4765(8) & 3.4(2) \\ \mathrm{C}(9) & -0.0765(8) & 0.0075(6) & 0.6404(6) & 2.5(2) \\ \mathrm{C}(10) & -0.0247(9) & -0.0406(5) & 0.7152(7) & 2.5(2) \\ \mathrm{C}(11) & -0.009(1) & 0.0044(9) & 0.7928(8) & 2.5(3) \\ \mathrm{C}(12) & -0.0438(9) & 0.0795(6) & 0.7677(7) & 2.7(2) \\ \mathrm{C}(13) & -0.0866(9) & 0.0830(6) & 0.6766(7) & 2.6(2) \\ \mathrm{C}(14) & 0.380(1) & -0.0633(7) & 0.7965(8) & 2.4(2) \\ \mathrm{C}(15) & 0.291(1) & 0.0101(6) & 0.8928(7) & 3.5(2) \\ \mathrm{C}(16) & 0.4307(9) & 0.2135(5) & 0.8090(6) & 2.2(2) \\ & & & \mathrm{S} 5 & \end{array}$




\begin{tabular}{|c|c|c|c|c|}
\hline $\mathrm{C}(17)$ & $0.507(1)$ & $0.1516(6)$ & $0.7979(7)$ & $3.0(2)$ \\
\hline $\mathrm{C}(18)$ & $0.6389(10)$ & $0.1609(7)$ & $0.8161(8)$ & $3.3(2)$ \\
\hline C(19) & $0.695(1)$ & $0.2297(6)$ & $0.8468(8)$ & $2.9(2)$ \\
\hline $\mathrm{C}(20)$ & $0.623(1)$ & $0.2904(6)$ & $0.8605(9)$ & $3.3(3)$ \\
\hline $\mathrm{C}(21)$ & $0.493(1)$ & $0.2818(7)$ & $0.8426(9)$ & $2.7(2)$ \\
\hline $\mathrm{C}(22)$ & $0.1945(9)$ & $0.2365(6)$ & $0.9045(7)$ & $2.6(2)$ \\
\hline $\mathrm{C}(23)$ & $0.284(1)$ & $0.2189(6)$ & $0.9865(7)$ & $3.0(2)$ \\
\hline $\mathrm{C}(24)$ & $0.260(1)$ & $0.2354(8)$ & $1.0685(8)$ & $2.3(3)$ \\
\hline$C(25)$ & $0.151(2)$ & $0.2703(9)$ & $1.0701(10)$ & $2.7(4)$ \\
\hline$C(26)$ & $0.063(1)$ & $0.2887(7)$ & $0.9926(9)$ & $4.1(3)$ \\
\hline $\mathrm{C}(27)$ & $0.0832(10)$ & $0.2720(6)$ & $0.9107(7)$ & $2.6(2)$ \\
\hline $\mathrm{C}(28)$ & $0.1650(9)$ & $0.3095(6)$ & $0.7015(7)$ & $2.5(2)$ \\
\hline C(29) & $0.1286(9)$ & $0.3121(6)$ & $0.6096(7)$ & $2.6(2)$ \\
\hline $\mathrm{C}(30)$ & $0.0987(10)$ & $0.3805(7)$ & $0.5617(7)$ & $3.2(2)$ \\
\hline $\mathrm{C}(31)$ & $0.1052(10)$ & $0.4494(6)$ & $0.6062(8)$ & $3.1(2)$ \\
\hline $\mathrm{C}(32)$ & $0.147(2)$ & $0.4503(5)$ & $0.700(1)$ & $3.0(2)$ \\
\hline $\mathrm{C}(33)$ & $0.174(1)$ & $0.3812(6)$ & $0.7464(8)$ & $2.7(2)$ \\
\hline$C(100)$ & 0.1688 & 0.0436 & 0.5739 & 0.2000 \\
\hline $\mathrm{C}(101)$ & -0.0482 & 0.0268 & 0.7185 & 0.2000 \\
\hline $\mathrm{H}(1)$ & 0.0318 & 0.1381 & 0.5269 & -0.0953 \\
\hline $\mathrm{H}(2)$ & 0.2931 & 0.1426 & 0.5846 & 1.4175 \\
\hline $\mathrm{H}(3)$ & 0.3479 & 0.0172 & 0.6135 & 3.0242 \\
\hline $\mathrm{H}(4)$ & 0.1862 & -0.0729 & 0.5941 & 0.7939 \\
\hline $\mathrm{H}(5)$ & -0.0314 & -0.1254 & 0.5676 & 3.6617 \\
\hline $\mathrm{H}(6)$ & -0.0712 & -0.1060 & 0.4663 & 3.6617 \\
\hline $\mathrm{H}(7)$ & -0.1730 & -0.1122 & 0.5180 & 3.6617 \\
\hline $\mathrm{H}(8)$ & -0.1722 & 0.0197 & 0.4173 & 4.1126 \\
\hline $\mathrm{H}(9)$ & -0.2547 & 0.0234 & 0.4837 & 4.1126 \\
\hline $\mathrm{H}(10)$ & -0.1581 & 0.0887 & 0.4832 & 4.1126 \\
\hline $\mathrm{H}(11)$ & -0.0125 & -0.0957 & 0.7208 & -0.8373 \\
\hline $\mathrm{H}(12)$ & 0.0317 & -0.0072 & 0.8365 & 0.1845 \\
\hline $\mathrm{H}(13)$ & -0.0445 & 0.1118 & 0.8059 & 2.0209 \\
\hline $\mathrm{H}(14)$ & -0.1124 & 0.1084 & 0.6437 & 1.8069 \\
\hline $\mathrm{H}(15)$ & 0.3747 & -0.0749 & 0.7355 & 2.8282 \\
\hline $\mathrm{H}(16)$ & 0.3638 & -0.1076 & 0.8260 & 2.8282 \\
\hline $\mathrm{H}(17)$ & 0.4615 & -0.0431 & 0.8248 & 2.8282 \\
\hline $\mathrm{H}(18)$ & 0.3097 & -0.0365 & 0.9269 & 4.1860 \\
\hline H(19) & 0.3544 & 0.0476 & 0.9180 & 4.1860 \\
\hline $\mathrm{H}(20)$ & 0.2109 & 0.0292 & 0.8964 & 4.1860 \\
\hline $\mathrm{H}(21)$ & 0.4717 & 0.1045 & 0.7800 & 3.6503 \\
\hline $\mathrm{H}(22)$ & 0.6880 & 0.1189 & 0.8043 & 3.9869 \\
\hline $\mathrm{H}(23)$ & 0.7832 & 0.2345 & 0.8590 & 3.4692 \\
\hline $\mathrm{H}(24)$ & 0.6625 & 0.3374 & 0.8837 & 3.9989 \\
\hline $\mathrm{H}(25)$ & 0.4429 & 0.3251 & 0.8509 & 3.2993 \\
\hline H(26) & 0.3627 & 0.1964 & 0.9861 & 3.5480 \\
\hline $\mathrm{H}(27)$ & 0.3214 & 0.2218 & 1.1229 & 2.8049 \\
\hline
\end{tabular}




$\begin{array}{lrrrr}\mathrm{H}(28) & 0.1340 & 0.2810 & 1.1256 & 3.2993 \\ \mathrm{H}(29) & -0.0135 & 0.3150 & 0.9942 & 4.8998 \\ \mathrm{H}(30) & 0.0197 & 0.2841 & 0.8576 & 3.1222 \\ \mathrm{H}(31) & 0.1244 & 0.2648 & 0.5787 & 3.1193 \\ \mathrm{H}(32) & 0.0738 & 0.3793 & 0.4976 & 3.8477 \\ \mathrm{H}(33) & 0.0833 & 0.4968 & 0.5741 & 3.7541 \\ \mathrm{H}(34) & 0.1539 & 0.4976 & 0.7319 & 3.5700 \\ \mathrm{H}(35) & 0.2005 & 0.3816 & 0.8098 & 3.2521\end{array}$

$B_{\text {eq }}=8 / 3 \pi^{2}\left(U_{11}\left(a a^{*}\right)^{2}+U_{22}\left(b b^{*}\right)^{2}+U_{33}\left(c c^{*}\right)^{2}+2 U_{12}\left(a a^{*} b b^{*}\right) \cos \gamma+2 U_{13}\left(a a^{*} c c^{*}\right) \cos \beta+\right.$ $\left.2 \mathrm{U}_{23}\left(\mathrm{bb}^{*} \mathrm{cc} *\right) \cos \alpha\right)$

Table 5. Anisotropic Displacement Parameters for $\left[\mathrm{Me}_{2} \mathrm{C}\left(\mathrm{C}_{5} \mathrm{H}_{4}\right)_{2}\right] \mathrm{Hf}\left(\mathrm{SnPh}_{3}\right) \mathrm{NMe}_{2}$ (2)

\begin{tabular}{|c|c|c|c|c|c|c|}
\hline atom & $\mathrm{U}_{11}$ & $\mathrm{U}_{22}$ & $\mathrm{U}_{33}$ & $\mathrm{U}_{12}$ & $\mathrm{U}_{13}$ & $\mathrm{U}_{23}$ \\
\hline $\operatorname{Sn}(1)$ & $0.0240(3)$ & $0.0247(3)$ & $0.0259(3)$ & $0.0002(3)$ & $0.0063(2)$ & $-0.0009(3)$ \\
\hline $\mathrm{N}(1)$ & $0.027(4)$ & $0.033(4)$ & $0.024(4)$ & $0.004(4)$ & $0.011(3)$ & $0.003(3)$ \\
\hline$C(1)$ & $0.037(5)$ & $0.033(5)$ & $0.027(5)$ & $0.005(5)$ & $0.012(4)$ & $-0.006(4)$ \\
\hline$C(2)$ & $0.034(5)$ & $0.036(5)$ & $0.029(5)$ & $0.000(5)$ & $0.016(4)$ & $-0.010(4)$ \\
\hline$C(3)$ & $0.021(5)$ & $0.069(8)$ & $0.032(6)$ & $-0.011(6)$ & $0.007(4)$ & $-0.014(6)$ \\
\hline $\mathrm{C}(4)$ & $0.031(5)$ & $0.024(5)$ & $0.036(5)$ & $-0.001(4)$ & $0.018(4)$ & $-0.003(4)$ \\
\hline $\mathrm{C}(5)$ & $0.021(5)$ & $0.052(7)$ & $0.022(5)$ & $-0.006(5)$ & $0.002(4)$ & $0.002(5)$ \\
\hline$C(6)$ & $0.022(5)$ & $0.056(7)$ & $0.028(5)$ & $0.001(5)$ & $0.009(4)$ & $-0.006(5)$ \\
\hline$C(7)$ & $0.038(6)$ & $0.048(7)$ & $0.041(6)$ & $-0.018(5)$ & $0.011(5)$ & $-0.019(5)$ \\
\hline $\mathrm{C}(8)$ & $0.025(5)$ & $0.065(8)$ & $0.036(6)$ & $-0.003(5)$ & $0.006(4)$ & $0.003(5)$ \\
\hline $\mathrm{C}(9)$ & $0.016(4)$ & $0.028(5)$ & $0.037(5)$ & $-0.001(4)$ & $0.014(4)$ & $-0.003(4)$ \\
\hline$C(10)$ & $0.027(4)$ & $0.026(5)$ & $0.032(5)$ & $0.005(4)$ & $0.010(4)$ & $0.009(4)$ \\
\hline $\mathrm{C}(11)$ & $0.032(6)$ & $0.061(9)$ & $0.031(6)$ & $0.008(6)$ & $0.009(5)$ & $0.013(6)$ \\
\hline$C(12)$ & $0.023(4)$ & $0.040(6)$ & $0.040(6)$ & $-0.006(4)$ & $0.015(4)$ & $-0.020(5)$ \\
\hline$C(13)$ & $0.026(5)$ & $0.032(6)$ & $0.033(5)$ & $0.010(4)$ & $0.004(4)$ & $-0.002(4)$ \\
\hline$C(14)$ & $0.038(6)$ & $0.041(6)$ & $0.044(7)$ & $0.007(5)$ & $0.010(5)$ & $0.006(5)$ \\
\hline$C(15)$ & $0.031(6)$ & $0.037(6)$ & $0.030(6)$ & $-0.011(5)$ & $-0.005(4)$ & $0.007(4)$ \\
\hline$C(16)$ & $0.033(5)$ & $0.024(5)$ & $0.027(5)$ & $0.009(4)$ & $0.013(4)$ & $0.009(4)$ \\
\hline$C(17)$ & $0.033(5)$ & $0.030(5)$ & $0.038(6)$ & $0.001(4)$ & $0.003(4)$ & $0.002(4)$ \\
\hline$C(18)$ & $0.023(5)$ & $0.038(6)$ & $0.058(7)$ & $-0.002(4)$ & $0.007(5)$ & $-0.005(5)$ \\
\hline$C(19)$ & $0.041(6)$ & $0.028(5)$ & $0.041(6)$ & $0.001(5)$ & $0.014(5)$ & $-0.008(5)$ \\
\hline$C(20)$ & $0.028(5)$ & $0.033(6)$ & $0.064(8)$ & $0.003(5)$ & $0.012(5)$ & $-0.008(5)$ \\
\hline $\mathrm{C}(21)$ & $0.026(5)$ & $0.027(6)$ & $0.061(8)$ & $0.001(5)$ & $0.013(5)$ & $-0.009(5)$ \\
\hline$C(22)$ & $0.032(5)$ & $0.025(5)$ & $0.035(5)$ & $-0.007(4)$ & $0.012(4)$ & $0.002(4)$ \\
\hline$C(23)$ & $0.048(6)$ & $0.027(5)$ & $0.032(6)$ & $0.002(5)$ & $0.009(5)$ & $-0.002(4)$ \\
\hline$C(24)$ & $0.063(8)$ & $0.041(7)$ & $0.025(5)$ & $-0.022(6)$ & $0.003(5)$ & $-0.008(5)$ \\
\hline$C(25)$ & $0.09(1)$ & $0.053(9)$ & $0.053(9)$ & $-0.015(8)$ & $0.047(9)$ & $-0.003(7)$ \\
\hline$C(26)$ & $0.056(7)$ & $0.048(7)$ & $0.054(8)$ & $-0.007(6)$ & $0.031(6)$ & $-0.019(6)$ \\
\hline$C(27)$ & $0.033(5)$ & $0.038(6)$ & $0.038(6)$ & $0.008(5)$ & $0.011(4)$ & $-0.001(5)$ \\
\hline$C(28)$ & $0.030(5)$ & $0.031(5)$ & $0.032(5)$ & $-0.001(4)$ & $0.012(4)$ & $-0.007(4)$ \\
\hline$C(29)$ & $0.033(5)$ & $0.024(5)$ & $0.040(6)$ & $0.000(4)$ & $0.008(4)$ & $-0.004(4)$ \\
\hline
\end{tabular}




$\begin{array}{lllllll}\mathrm{C}(30) & 0.030(5) & 0.050(7) & 0.032(5) & -0.001(5) & 0.006(4) & 0.014(5) \\ \mathrm{C}(31) & 0.023(5) & 0.045(7) & 0.052(7) & 0.008(5) & 0.012(5) & 0.008(5) \\ \mathrm{C}(32) & 0.030(5) & 0.036(5) & 0.056(9) & 0.006(5) & 0.021(5) & 0.004(6) \\ \mathrm{C}(33) & 0.039(5) & 0.025(5) & 0.040(5) & 0.007(4) & 0.011(4) & 0.007(4)\end{array}$

The general temperature factor expression:

$\exp \left(-2 \pi^{2}\left(a^{*}{ }^{2} U_{11} h^{2}+b^{* 2} U_{22} k^{2}+c^{* 2} U_{33} l^{2}+2 a * b * U_{12} h k+2 a * c * U_{13} h l+2 b * c * U_{23} k l\right)\right)$

Table 6. Bond Lengths $(\AA)$ for $\left[\mathrm{Me}_{2} \mathrm{C}\left(\mathrm{C}_{5} \mathrm{H}_{4}\right)_{2}\right] \mathrm{Hf}\left(\mathrm{SnPh}_{3}\right) \mathrm{NMe}_{2}$ (2)

\begin{tabular}{|c|c|c|c|c|c|}
\hline atom & atom & distance & atom & atom & distance \\
\hline $\mathrm{Hf}(1)$ & $\operatorname{Sn}(1)$ & $2.9428(7)$ & $\operatorname{Hf}(1)$ & $\mathrm{N}(1)$ & $2.057(8)$ \\
\hline $\mathrm{Hf}(1)$ & $\mathrm{C}(1)$ & $2.523(10)$ & $\operatorname{Hf}(1)$ & $\mathrm{C}(2)$ & $2.624(9)$ \\
\hline $\operatorname{Hf}(1)$ & $\mathrm{C}(3)$ & $2.59(1)$ & $\operatorname{Hf}(1)$ & $\mathrm{C}(4)$ & $2.474(9)$ \\
\hline $\mathrm{Hf}(1)$ & $C(5)$ & $2.46(1)$ & $\mathrm{Hf}(1)$ & $\mathrm{C}(9)$ & $2.418(9)$ \\
\hline $\mathrm{Hf}(1)$ & $C(10)$ & $2.394(9)$ & $\operatorname{Hf}(1)$ & $\mathrm{C}(11)$ & $2.47(1)$ \\
\hline $\mathrm{Hf}(1)$ & $C(12)$ & $2.469(8)$ & $\operatorname{Hf}(1)$ & $\mathrm{C}(13)$ & $2.450(9)$ \\
\hline $\mathrm{Hf}(1)$ & $\mathrm{C}(100)$ & 2.22 & $\operatorname{Hf}(1)$ & $\mathrm{C}(101)$ & $2.12860(10)$ \\
\hline $\operatorname{Sn}(1)$ & $\mathrm{C}(16)$ & $2.195(10)$ & $\operatorname{Sn}(1)$ & $\mathrm{C}(22)$ & $2.147(10)$ \\
\hline $\operatorname{Sn}(1)$ & $C(28)$ & $2.17(1)$ & $\mathrm{N}(1)$ & $\mathrm{C}(14)$ & $1.44(1)$ \\
\hline $\mathrm{N}(1)$ & $C(15)$ & $1.48(1)$ & $\mathrm{C}(1)$ & $C(2)$ & $1.44(1)$ \\
\hline $\mathrm{C}(1)$ & $C(5)$ & $1.42(2)$ & $\mathrm{C}(1)$ & $\mathrm{C}(100)$ & $1.20(1)$ \\
\hline $\mathrm{C}(2)$ & $\mathrm{C}(3)$ & $1.43(2)$ & $\mathrm{C}(2)$ & $C(100)$ & $1.23(1)$ \\
\hline $\mathrm{C}(3)$ & $\mathrm{C}(4)$ & $1.43(2)$ & $\mathrm{C}(3)$ & $\mathrm{C}(100)$ & $1.21(1)$ \\
\hline $\mathrm{C}(4)$ & $C(5)$ & $1.43(1)$ & $C(4)$ & $C(100)$ & $1.211(10)$ \\
\hline$C(5)$ & $C(6)$ & $1.53(1)$ & $\mathrm{C}(5)$ & $\mathrm{C}(100)$ & $1.226(10)$ \\
\hline$C(6)$ & $\mathrm{C}(7)$ & $1.56(2)$ & $\mathrm{C}(6)$ & $\mathrm{C}(8)$ & $1.49(2)$ \\
\hline $\mathrm{C}(6)$ & $\mathrm{C}(9)$ & $1.53(1)$ & $\mathrm{C}(9)$ & $\mathrm{C}(10)$ & $1.42(1)$ \\
\hline $\mathrm{C}(9)$ & $\mathrm{C}(13)$ & $1.44(1)$ & $\mathrm{C}(9)$ & $\mathrm{C}(101)$ & $1.214(9)$ \\
\hline$C(10)$ & $\mathrm{C}(11)$ & $1.41(2)$ & $\mathrm{C}(10)$ & $\mathrm{C}(101)$ & $1.198(9)$ \\
\hline$C(11)$ & $C(12)$ & $1.38(2)$ & $\mathrm{C}(11)$ & $\mathrm{C}(101)$ & $1.18(1)$ \\
\hline$C(12)$ & $C(13)$ & $1.36(1)$ & $\mathrm{C}(12)$ & $\mathrm{C}(101)$ & 1.181(9) \\
\hline$C(13)$ & $\mathrm{C}(101)$ & $1.18(1)$ & $\mathrm{C}(16)$ & $\mathrm{C}(17)$ & $1.40(1)$ \\
\hline$C(16)$ & $\mathrm{C}(21)$ & $1.39(1)$ & $\mathrm{C}(17)$ & $\mathrm{C}(18)$ & $1.40(1)$ \\
\hline $\mathrm{C}(18)$ & $C(19)$ & $1.36(1)$ & $\mathrm{C}(19)$ & $\mathrm{C}(20)$ & $1.37(1)$ \\
\hline$C(20)$ & $\mathrm{C}(21)$ & $1.38(2)$ & $\mathrm{C}(22)$ & $\mathrm{C}(23)$ & $1.41(2)$ \\
\hline$C(22)$ & $C(27)$ & $1.39(1)$ & $\mathrm{C}(23)$ & $\mathrm{C}(24)$ & $1.40(2)$ \\
\hline $\mathrm{C}(24)$ & $C(25)$ & $1.35(2)$ & $\mathrm{C}(25)$ & $C(26)$ & $1.36(2)$ \\
\hline$C(26)$ & $C(27)$ & $1.38(2)$ & $\mathrm{C}(28)$ & $C(29)$ & $1.37(2)$ \\
\hline $\mathrm{C}(28)$ & $C(33)$ & $1.41(1)$ & $\mathrm{C}(29)$ & $\mathrm{C}(30)$ & $1.39(1)$ \\
\hline$C(30)$ & $\mathrm{C}(31)$ & $1.37(2)$ & $\mathrm{C}(31)$ & $\mathrm{C}(32)$ & $1.40(2)$ \\
\hline$C(32)$ & $C(33)$ & $1.38(2)$ & & & \\
\hline
\end{tabular}

Table 7. Bond Angles $\left({ }^{\mathrm{O}}\right)$ for $\left[\mathrm{Me}_{2} \mathrm{C}\left(\mathrm{C}_{5} \mathrm{H}_{4}\right)_{2}\right] \mathrm{Hf}\left(\mathrm{SnPh}_{3}\right) \mathrm{NMe}_{2}$ (2) 


\begin{tabular}{|c|c|c|c|c|c|c|c|}
\hline atom & atom & atom & angle & atom & atom & atom & angle \\
\hline $\operatorname{Sn}(1)$ & $\operatorname{Hf}(1)$ & $\mathrm{N}(1)$ & $93.3(2)$ & $\operatorname{Sn}(1)$ & $\mathrm{Hf}(1)$ & $\mathrm{C}(1)$ & $90.9(2)$ \\
\hline $\operatorname{Sn}(1)$ & $\operatorname{Hf}(1)$ & $\mathrm{C}(2)$ & $78.3(2)$ & $\operatorname{Sn}(1)$ & $\operatorname{Hf}(1)$ & $\mathrm{C}(3)$ & $100.8(3)$ \\
\hline $\operatorname{Sn}(1)$ & $\operatorname{Hf}(1)$ & $\mathrm{C}(4)$ & $131.6(2)$ & $\operatorname{Sn}(1)$ & $\operatorname{Hf}(1)$ & $C(5)$ & $123.9(3)$ \\
\hline $\operatorname{Sn}(1)$ & $\operatorname{Hf}(1)$ & $\mathrm{C}(9)$ & $127.6(2)$ & $\operatorname{Sn}(1)$ & $\mathrm{Hf}(1)$ & $\mathrm{C}(10)$ & $138.2(2)$ \\
\hline $\operatorname{Sn}(1)$ & $\operatorname{Hf}(1)$ & $\mathrm{C}(11)$ & $106.7(3)$ & $\operatorname{Sn}(1)$ & $\mathrm{Hf}(1)$ & $\mathrm{C}(12)$ & $83.4(2)$ \\
\hline $\operatorname{Sn}(1)$ & $\operatorname{Hf}(1)$ & $\mathrm{C}(13)$ & $93.6(2)$ & $\operatorname{Sn}(1)$ & $\operatorname{Hf}(1)$ & $\mathrm{C}(100)$ & $105.65(2)$ \\
\hline $\operatorname{Sn}(1)$ & $\operatorname{Hf}(1)$ & $\mathrm{C}(101)$ & $111.12(2)$ & $\mathrm{N}(1)$ & $\operatorname{Hf}(1)$ & $\mathrm{C}(1)$ & $134.5(3)$ \\
\hline $\mathrm{N}(1)$ & $\operatorname{Hf}(1)$ & $C(2)$ & $105.0(3)$ & $\mathrm{N}(1)$ & $\operatorname{Hf}(1)$ & $\mathrm{C}(3)$ & $81.5(4)$ \\
\hline $\mathrm{N}(1)$ & $\operatorname{Hf}(1)$ & $C(4)$ & $91.1(3)$ & $\mathrm{N}(1)$ & $\operatorname{Hf}(1)$ & $C(5)$ & $124.6(3)$ \\
\hline $\mathrm{N}(1)$ & $\operatorname{Hf}(1)$ & $\mathrm{C}(9)$ & $131.2(3)$ & $\mathrm{N}(1)$ & $\mathrm{Hf}(1)$ & $\mathrm{C}(10)$ & $98.0(3)$ \\
\hline $\mathrm{N}(1)$ & $\operatorname{Hf}(1)$ & $\mathrm{C}(11)$ & $91.0(4)$ & $\mathrm{N}(1)$ & $\operatorname{Hf}(1)$ & $C(12)$ & $115.0(3)$ \\
\hline $\mathrm{N}(1)$ & $\operatorname{Hf}(1)$ & $\mathrm{C}(13)$ & $144.8(3)$ & $\mathrm{N}(1)$ & $\mathrm{Hf}(1)$ & $\mathrm{C}(100)$ & $108.4(2)$ \\
\hline $\mathrm{N}(1)$ & $\operatorname{Hf}(1)$ & $\mathrm{C}(101)$ & $118.1(2)$ & $\mathrm{C}(1)$ & $\operatorname{Hf}(1)$ & $\mathrm{C}(2)$ & $32.4(3)$ \\
\hline $\mathrm{C}(1)$ & $\operatorname{Hf}(1)$ & $\mathrm{C}(3)$ & $53.3(4)$ & $\mathrm{C}(1)$ & $\mathrm{Hf}(1)$ & $\mathrm{C}(4)$ & $54.5(3)$ \\
\hline$C(1)$ & $\operatorname{Hf}(1)$ & $C(5)$ & $33.0(4)$ & $\mathrm{C}(1)$ & $\operatorname{Hf}(1)$ & $\mathrm{C}(9)$ & $76.9(3)$ \\
\hline $\mathrm{C}(1)$ & $\operatorname{Hf}(1)$ & $\mathrm{C}(10)$ & $108.3(3)$ & $\mathrm{C}(1)$ & $\operatorname{Hf}(1)$ & $\mathrm{C}(11)$ & $130.7(4)$ \\
\hline $\mathrm{C}(1)$ & $\operatorname{Hf}(1)$ & $\mathrm{C}(12)$ & $110.4(3)$ & $\mathrm{C}(1)$ & $\operatorname{Hf}(1)$ & $C(13)$ & $79.8(3)$ \\
\hline $\mathrm{C}(1)$ & $\operatorname{Hf}(1)$ & $\mathrm{C}(100)$ & $28.3(2)$ & $\mathrm{C}(1)$ & $\mathrm{Hf}(1)$ & $\mathrm{C}(101)$ & $102.2(2)$ \\
\hline$C(2)$ & $\operatorname{Hf}(1)$ & $\mathrm{C}(3)$ & $31.8(4)$ & $\mathrm{C}(2)$ & $\operatorname{Hf}(1)$ & $\mathrm{C}(4)$ & $54.1(3)$ \\
\hline$C(2)$ & $\operatorname{Hf}(1)$ & $\mathrm{C}(5)$ & $54.7(3)$ & $\mathrm{C}(2)$ & $\operatorname{Hf}(1)$ & $\mathrm{C}(9)$ & $108.3(3)$ \\
\hline$C(2)$ & $\operatorname{Hf}(1)$ & $\mathrm{C}(10)$ & $135.4(3)$ & $\mathrm{C}(2)$ & $\mathrm{Hf}(1)$ & $\mathrm{C}(11)$ & $163.1(4)$ \\
\hline$C(2)$ & $\operatorname{Hf}(1)$ & $\mathrm{C}(12)$ & $136.7(3)$ & $C(2)$ & $\mathrm{Hf}(1)$ & $\mathrm{C}(13)$ & $110.2(3)$ \\
\hline $\mathrm{C}(2)$ & $\operatorname{Hf}(1)$ & $\mathrm{C}(100)$ & $27.9(2)$ & $\mathrm{C}(2)$ & $\mathrm{Hf}(1)$ & $\mathrm{C}(101)$ & $134.5(2)$ \\
\hline $\mathrm{C}(3)$ & $\operatorname{Hf}(1)$ & $\mathrm{C}(4)$ & $32.7(4)$ & $\mathrm{C}(3)$ & $\operatorname{Hf}(1)$ & $C(5)$ & $54.6(3)$ \\
\hline $\mathrm{C}(3)$ & $\operatorname{Hf}(1)$ & $\mathrm{C}(9)$ & $110.7(3)$ & $\mathrm{C}(3)$ & $\operatorname{Hf}(1)$ & $C(10)$ & $120.6(4)$ \\
\hline$C(3)$ & $\operatorname{Hf}(1)$ & $\mathrm{C}(11)$ & $151.9(4)$ & $\mathrm{C}(3)$ & $\operatorname{Hf}(1)$ & $C(12)$ & $162.9(4)$ \\
\hline$C(3)$ & $\operatorname{Hf}(1)$ & $\mathrm{C}(13)$ & $130.7(4)$ & $\mathrm{C}(3)$ & $\mathrm{Hf}(1)$ & $\mathrm{C}(100)$ & $27.8(3)$ \\
\hline $\mathrm{C}(3)$ & $\operatorname{Hf}(1)$ & $\mathrm{C}(101)$ & $140.4(3)$ & $\mathrm{C}(4)$ & $\operatorname{Hf}(1)$ & $\mathrm{C}(5)$ & $33.7(3)$ \\
\hline$C(4)$ & $\operatorname{Hf}(1)$ & $\mathrm{C}(9)$ & $80.8(3)$ & $\mathrm{C}(4)$ & $\mathrm{Hf}(1)$ & $C(10)$ & $88.4(3)$ \\
\hline $\mathrm{C}(4)$ & $\operatorname{Hf}(1)$ & $\mathrm{C}(11)$ & $121.5(4)$ & $\mathrm{C}(4)$ & $\mathrm{Hf}(1)$ & $C(12)$ & $136.4(3)$ \\
\hline $\mathrm{C}(4)$ & $\mathrm{Hf}(1)$ & $\mathrm{C}(13)$ & $109.4(3)$ & $\mathrm{C}(4)$ & $\mathrm{Hf}(1)$ & $\mathrm{C}(100)$ & $29.3(2)$ \\
\hline $\mathrm{C}(4)$ & $\operatorname{Hf}(1)$ & $\mathrm{C}(101)$ & $108.8(2)$ & $C(5)$ & $\operatorname{Hf}(1)$ & $\mathrm{C}(9)$ & $57.4(3)$ \\
\hline$C(5)$ & $\operatorname{Hf}(1)$ & $\mathrm{C}(10)$ & $80.8(3)$ & $C(5)$ & $\operatorname{Hf}(1)$ & $\mathrm{C}(11)$ & $111.6(4)$ \\
\hline$C(5)$ & $\operatorname{Hf}(1)$ & $\mathrm{C}(12)$ & 109.2(3) & $C(5)$ & $\operatorname{Hf}(1)$ & $C(13)$ & $78.1(3)$ \\
\hline$C(5)$ & $\operatorname{Hf}(1)$ & $\mathrm{C}(100)$ & $29.8(2)$ & $C(5)$ & $\operatorname{Hf}(1)$ & $\mathrm{C}(101)$ & $87.5(2)$ \\
\hline $\mathrm{C}(9)$ & $\operatorname{Hf}(1)$ & $C(10)$ & $34.2(3)$ & $\mathrm{C}(9)$ & $\operatorname{Hf}(1)$ & $\mathrm{C}(11)$ & $55.6(4)$ \\
\hline $\mathrm{C}(9)$ & $\mathrm{Hf}(1)$ & $\mathrm{C}(12)$ & $55.6(3)$ & $\mathrm{C}(9)$ & $\operatorname{Hf}(1)$ & $\mathrm{C}(13)$ & $34.3(3)$ \\
\hline $\mathrm{C}(9)$ & $\operatorname{Hf}(1)$ & $\mathrm{C}(100)$ & $87.2(2)$ & $\mathrm{C}(9)$ & $\operatorname{Hf}(1)$ & $\mathrm{C}(101)$ & $30.1(2)$ \\
\hline$C(10)$ & $\operatorname{Hf}(1)$ & $\mathrm{C}(11)$ & $33.6(4)$ & $C(10)$ & $\mathrm{Hf}(1)$ & $\mathrm{C}(12)$ & $55.4(3)$ \\
\hline$C(10)$ & $\operatorname{Hf}(1)$ & $\mathrm{C}(13)$ & $55.9(3)$ & $C(10)$ & $\operatorname{Hf}(1)$ & $\mathrm{C}(100)$ & $108.5(2)$ \\
\hline$C(10)$ & $\operatorname{Hf}(1)$ & $\mathrm{C}(101)$ & $30.0(2)$ & $\mathrm{C}(11)$ & $\operatorname{Hf}(1)$ & $C(12)$ & $32.4(4)$ \\
\hline $\mathrm{C}(11)$ & $\operatorname{Hf}(1)$ & $\mathrm{C}(13)$ & $54.0(4)$ & $\mathrm{C}(11)$ & $\operatorname{Hf}(1)$ & $\mathrm{C}(100)$ & $141.0(3)$ \\
\hline $\mathrm{C}(11)$ & $\operatorname{Hf}(1)$ & $\mathrm{C}(101)$ & $28.6(3)$ & $\mathrm{C}(12)$ & $\operatorname{Hf}(1)$ & $C(13)$ & $32.2(3)$ \\
\hline$C(12)$ & $\operatorname{Hf}(1)$ & $\mathrm{C}(100)$ & $135.0(3)$ & $C(12)$ & $\operatorname{Hf}(1)$ & $\mathrm{C}(101)$ & $28.6(2)$ \\
\hline
\end{tabular}




\begin{tabular}{|c|c|c|c|c|c|c|c|}
\hline $\mathrm{C}(13)$ & $\operatorname{Hf}(1)$ & $\mathrm{C}(100)$ & $102.8(2)$ & $\mathrm{C}(13)$ & $\operatorname{Hf}(1)$ & $\mathrm{C}(101)$ & $28.8(2)$ \\
\hline$C(100)$ & $\operatorname{Hf}(1)$ & $\mathrm{C}(101)$ & $117.077(3)$ & $\mathrm{Hf}(1)$ & $\operatorname{Sn}(1)$ & $C(16)$ & $110.6(2)$ \\
\hline $\operatorname{Hf}(1)$ & $\operatorname{Sn}(1)$ & $\mathrm{C}(22)$ & $116.9(2)$ & $\mathrm{Hf}(1)$ & $\operatorname{Sn}(1)$ & $\mathrm{C}(28)$ & $123.0(3)$ \\
\hline$C(16)$ & $\operatorname{Sn}(1)$ & $\mathrm{C}(22)$ & $101.0(3)$ & $C(16)$ & $\operatorname{Sn}(1)$ & $\mathrm{C}(28)$ & 101.2(3) \\
\hline $\mathrm{C}(22)$ & $\operatorname{Sn}(1)$ & $\mathrm{C}(28)$ & $101.0(4)$ & $\mathrm{Hf}(1)$ & $\mathrm{N}(1)$ & $\mathrm{C}(14)$ & 138.1(7) \\
\hline $\mathrm{Hf}(1)$ & $\mathrm{N}(1)$ & $\mathrm{C}(15)$ & $112.9(6)$ & $C(14)$ & $\mathrm{N}(1)$ & $\mathrm{C}(15)$ & $108.3(8)$ \\
\hline $\mathrm{Hf}(1)$ & $\mathrm{C}(1)$ & $\mathrm{C}(2)$ & $77.7(6)$ & $\mathrm{Hf}(1)$ & $\mathrm{C}(1)$ & $\mathrm{C}(5)$ & $70.9(6)$ \\
\hline $\mathrm{Hf}(1)$ & $\mathrm{C}(1)$ & $\mathrm{C}(100)$ & $61.8(4)$ & $\mathrm{C}(2)$ & $\mathrm{C}(1)$ & $\mathrm{C}(5)$ & 109.9(9) \\
\hline $\mathrm{C}(2)$ & $\mathrm{C}(1)$ & $\mathrm{C}(100)$ & $54.7(6)$ & $\mathrm{C}(5)$ & $\mathrm{C}(1)$ & $\mathrm{C}(100)$ & $55.2(6)$ \\
\hline $\mathrm{Hf}(1)$ & $\mathrm{C}(2)$ & $\mathrm{C}(1)$ & $69.9(5)$ & $\mathrm{Hf}(1)$ & $\mathrm{C}(2)$ & $\mathrm{C}(3)$ & $72.7(6)$ \\
\hline $\mathrm{Hf}(1)$ & $\mathrm{C}(2)$ & $\mathrm{C}(100)$ & $57.6(4)$ & $\mathrm{C}(1)$ & $\mathrm{C}(2)$ & $\mathrm{C}(3)$ & $106.2(10)$ \\
\hline $\mathrm{C}(1)$ & $\mathrm{C}(2)$ & $\mathrm{C}(100)$ & $52.6(6)$ & $\mathrm{C}(3)$ & $\mathrm{C}(2)$ & $\mathrm{C}(100)$ & $53.6(6)$ \\
\hline $\mathrm{Hf}(1)$ & $\mathrm{C}(3)$ & $\mathrm{C}(2)$ & $75.5(6)$ & $\mathrm{Hf}(1)$ & $\mathrm{C}(3)$ & $\mathrm{C}(4)$ & $69.2(5)$ \\
\hline $\mathrm{Hf}(1)$ & $\mathrm{C}(3)$ & $\mathrm{C}(100)$ & $59.0(4)$ & $\mathrm{C}(2)$ & $\mathrm{C}(3)$ & $\mathrm{C}(4)$ & $108.7(10)$ \\
\hline $\mathrm{C}(2)$ & $\mathrm{C}(3)$ & $\mathrm{C}(100)$ & $54.9(7)$ & $\mathrm{C}(4)$ & $\mathrm{C}(3)$ & $\mathrm{C}(100)$ & $53.8(6)$ \\
\hline $\mathrm{Hf}(1)$ & $\mathrm{C}(4)$ & $\mathrm{C}(3)$ & $78.1(6)$ & $\operatorname{Hf}(1)$ & $\mathrm{C}(4)$ & $\mathrm{C}(5)$ & $72.5(5)$ \\
\hline $\mathrm{Hf}(1)$ & $\mathrm{C}(4)$ & $\mathrm{C}(100)$ & $63.8(4)$ & $\mathrm{C}(3)$ & $\mathrm{C}(4)$ & $C(5)$ & $108.3(9)$ \\
\hline$C(3)$ & $\mathrm{C}(4)$ & $\mathrm{C}(100)$ & $53.8(7)$ & $\mathrm{C}(5)$ & $\mathrm{C}(4)$ & $\mathrm{C}(100)$ & $54.5(6)$ \\
\hline $\operatorname{Hf}(1)$ & $C(5)$ & $\mathrm{C}(1)$ & $76.1(6)$ & $\mathrm{Hf}(1)$ & $\mathrm{C}(5)$ & $\mathrm{C}(4)$ & $73.8(6)$ \\
\hline $\operatorname{Hf}(1)$ & $\mathrm{C}(5)$ & $C(6)$ & $100.5(6)$ & $\mathrm{Hf}(1)$ & $\mathrm{C}(5)$ & $\mathrm{C}(100)$ & $64.5(4)$ \\
\hline $\mathrm{C}(1)$ & $\mathrm{C}(5)$ & $\mathrm{C}(4)$ & $106.9(8)$ & $\mathrm{C}(1)$ & $\mathrm{C}(5)$ & $\mathrm{C}(6)$ & $124.1(10)$ \\
\hline$C(1)$ & $C(5)$ & $\mathrm{C}(100)$ & $53.4(6)$ & $\mathrm{C}(4)$ & $\mathrm{C}(5)$ & $\mathrm{C}(6)$ & $125.9(10)$ \\
\hline $\mathrm{C}(4)$ & $C(5)$ & $\mathrm{C}(100)$ & $53.6(6)$ & $C(6)$ & $\mathrm{C}(5)$ & $\mathrm{C}(100)$ & $164.9(9)$ \\
\hline $\mathrm{C}(5)$ & $\mathrm{C}(6)$ & $\mathrm{C}(7)$ & $110.6(8)$ & $\mathrm{C}(5)$ & $\mathrm{C}(6)$ & $\mathrm{C}(8)$ & 111.9(9) \\
\hline$C(5)$ & $\mathrm{C}(6)$ & $\mathrm{C}(9)$ & $99.6(7)$ & $C(7)$ & $\mathrm{C}(6)$ & $\mathrm{C}(8)$ & 109.7(9) \\
\hline$C(7)$ & $C(6)$ & $\mathrm{C}(9)$ & $111.8(9)$ & $\mathrm{C}(8)$ & $C(6)$ & $\mathrm{C}(9)$ & $112.8(8)$ \\
\hline $\mathrm{Hf}(1)$ & $\mathrm{C}(9)$ & $\mathrm{C}(6)$ & $102.2(5)$ & $\mathrm{Hf}(1)$ & $\mathrm{C}(9)$ & $\mathrm{C}(10)$ & $72.0(5)$ \\
\hline $\mathrm{Hf}(1)$ & $\mathrm{C}(9)$ & $\mathrm{C}(13)$ & $74.1(5)$ & $\mathrm{Hf}(1)$ & $\mathrm{C}(9)$ & $\mathrm{C}(101)$ & $61.7(4)$ \\
\hline$C(6)$ & $\mathrm{C}(9)$ & $\mathrm{C}(10)$ & $125.5(9)$ & $C(6)$ & $\mathrm{C}(9)$ & $\mathrm{C}(13)$ & $125.3(9)$ \\
\hline$C(6)$ & $\mathrm{C}(9)$ & $\mathrm{C}(101)$ & $163.8(8)$ & $C(10)$ & $\mathrm{C}(9)$ & $\mathrm{C}(13)$ & $105.6(8)$ \\
\hline$C(10)$ & $\mathrm{C}(9)$ & $\mathrm{C}(101)$ & $53.5(5)$ & $\mathrm{C}(13)$ & $\mathrm{C}(9)$ & $\mathrm{C}(101)$ & $52.1(5)$ \\
\hline $\operatorname{Hf}(1)$ & $C(10)$ & $\mathrm{C}(9)$ & $73.8(5)$ & $\mathrm{Hf}(1)$ & $\mathrm{C}(10)$ & $\mathrm{C}(11)$ & $76.1(7)$ \\
\hline $\mathrm{Hf}(1)$ & $C(10)$ & $\mathrm{C}(101)$ & $62.7(4)$ & $\mathrm{C}(9)$ & $\mathrm{C}(10)$ & $\mathrm{C}(11)$ & $107.8(9)$ \\
\hline $\mathrm{C}(9)$ & $C(10)$ & $\mathrm{C}(101)$ & $54.6(5)$ & $\mathrm{C}(11)$ & $\mathrm{C}(10)$ & $\mathrm{C}(101)$ & $53.2(7)$ \\
\hline $\mathrm{Hf}(1)$ & $\mathrm{C}(11)$ & $\mathrm{C}(10)$ & $70.3(6)$ & $\mathrm{Hf}(1)$ & $\mathrm{C}(11)$ & $\mathrm{C}(12)$ & $73.8(6)$ \\
\hline $\mathrm{Hf}(1)$ & $\mathrm{C}(11)$ & $\mathrm{C}(101)$ & $59.6(5)$ & $C(10)$ & $\mathrm{C}(11)$ & $\mathrm{C}(12)$ & $108.6(10)$ \\
\hline$C(10)$ & $\mathrm{C}(11)$ & $\mathrm{C}(101)$ & $54.3(7)$ & $\mathrm{C}(12)$ & $\mathrm{C}(11)$ & $\mathrm{C}(101)$ & $54.3(7)$ \\
\hline $\mathrm{Hf}(1)$ & $C(12)$ & $\mathrm{C}(11)$ & $73.8(6)$ & $\operatorname{Hf}(1)$ & $\mathrm{C}(12)$ & $\mathrm{C}(13)$ & $73.1(5)$ \\
\hline $\mathrm{Hf}(1)$ & $\mathrm{C}(12)$ & $\mathrm{C}(101)$ & $59.5(3)$ & $\mathrm{C}(11)$ & $\mathrm{C}(12)$ & $\mathrm{C}(13)$ & $109.0(10)$ \\
\hline $\mathrm{C}(11)$ & $\mathrm{C}(12)$ & $\mathrm{C}(101)$ & $54.3(7)$ & $\mathrm{C}(13)$ & $C(12)$ & $\mathrm{C}(101)$ & $54.7(6)$ \\
\hline $\mathrm{Hf}(1)$ & $\mathrm{C}(13)$ & $\mathrm{C}(9)$ & $71.6(5)$ & $\mathrm{Hf}(1)$ & $\mathrm{C}(13)$ & $\mathrm{C}(12)$ & $74.7(5)$ \\
\hline $\mathrm{Hf}(1)$ & $\mathrm{C}(13)$ & $\mathrm{C}(101)$ & $60.3(4)$ & $\mathrm{C}(9)$ & $\mathrm{C}(13)$ & $\mathrm{C}(12)$ & $108.9(9)$ \\
\hline $\mathrm{C}(9)$ & $\mathrm{C}(13)$ & $\mathrm{C}(101)$ & $54.2(6)$ & $\mathrm{C}(12)$ & $\mathrm{C}(13)$ & $\mathrm{C}(101)$ & $54.7(6)$ \\
\hline $\operatorname{Sn}(1)$ & $C(16)$ & $\mathrm{C}(17)$ & $122.7(7)$ & $\operatorname{Sn}(1)$ & $C(16)$ & $\mathrm{C}(21)$ & $120.9(7)$ \\
\hline$C(17)$ & $C(16)$ & $\mathrm{C}(21)$ & 116.4(9) & $C(16)$ & $\mathrm{C}(17)$ & $\mathrm{C}(18)$ & $120.5(10)$ \\
\hline$C(17)$ & $\mathrm{C}(18)$ & $\mathrm{C}(19)$ & $120(1)$ & $\mathrm{C}(18)$ & $\mathrm{C}(19)$ & $\mathrm{C}(20)$ & 119(1) \\
\hline
\end{tabular}




$\begin{array}{llllllll}\mathrm{C}(19) & \mathrm{C}(20) & \mathrm{C}(21) & 119(1) & \mathrm{C}(16) & \mathrm{C}(21) & \mathrm{C}(20) & 122.6(10) \\ \mathrm{Sn}(1) & \mathrm{C}(22) & \mathrm{C}(23) & 121.9(7) & \mathrm{Sn}(1) & \mathrm{C}(22) & \mathrm{C}(27) & 122.2(8) \\ \mathrm{C}(23) & \mathrm{C}(22) & \mathrm{C}(27) & 115.9(9) & \mathrm{C}(22) & \mathrm{C}(23) & \mathrm{C}(24) & 121(1) \\ \mathrm{C}(23) & \mathrm{C}(24) & \mathrm{C}(25) & 119(1) & \mathrm{C}(24) & \mathrm{C}(25) & \mathrm{C}(26) & 120(1) \\ \mathrm{C}(25) & \mathrm{C}(26) & \mathrm{C}(27) & 120(1) & \mathrm{C}(22) & \mathrm{C}(27) & \mathrm{C}(26) & 121(1) \\ \mathrm{Sn}(1) & \mathrm{C}(28) & \mathrm{C}(29) & 123.8(7) & \mathrm{Sn}(1) & \mathrm{C}(28) & \mathrm{C}(33) & 119.5(8) \\ \mathrm{C}(29) & \mathrm{C}(28) & \mathrm{C}(33) & 116.5(9) & \mathrm{C}(28) & \mathrm{C}(29) & \mathrm{C}(30) & 122.9(9) \\ \mathrm{C}(29) & \mathrm{C}(30) & \mathrm{C}(31) & 119.9(10) & \mathrm{C}(30) & \mathrm{C}(31) & \mathrm{C}(32) & 119.4(10) \\ \mathrm{C}(31) & \mathrm{C}(32) & \mathrm{C}(33) & 119(1) & \mathrm{C}(28) & \mathrm{C}(33) & \mathrm{C}(32) & 121(1) \\ \mathrm{Hf}(1) & \mathrm{C}(100) & \mathrm{C}(1) & 89.9(4) & \mathrm{Hf}(1) & \mathrm{C}(100) & \mathrm{C}(2) & 94.5(4) \\ \mathrm{Hf}(1) & \mathrm{C}(100) & \mathrm{C}(3) & 93.1(5) & \mathrm{Hf}(1) & \mathrm{C}(100) & \mathrm{C}(4) & 86.9(4) \\ \mathrm{Hf}(1) & \mathrm{C}(100) & \mathrm{C}(5) & 85.7(4) & \mathrm{C}(1) & \mathrm{C}(100) & \mathrm{C}(2) & 72.8(7) \\ \mathrm{C}(1) & \mathrm{C}(100) & \mathrm{C}(3) & 144.3(9) & \mathrm{C}(1) & \mathrm{C}(100) & \mathrm{C}(4) & 143.3(7) \\ \mathrm{C}(1) & \mathrm{C}(100) & \mathrm{C}(5) & 71.4(7) & \mathrm{C}(2) & \mathrm{C}(100) & \mathrm{C}(3) & 71.6(8) \\ \mathrm{C}(2) & \mathrm{C}(100) & \mathrm{C}(4) & 143.9(7) & \mathrm{C}(2) & \mathrm{C}(100) & \mathrm{C}(5) & 144.2(7) \\ \mathrm{C}(3) & \mathrm{C}(100) & \mathrm{C}(4) & 72.4(8) & \mathrm{C}(3) & \mathrm{C}(100) & \mathrm{C}(5) & 144.2(9) \\ \mathrm{C}(4) & \mathrm{C}(100) & \mathrm{C}(5) & 71.9(7) & \mathrm{Hf}(1) & \mathrm{C}(101) & \mathrm{C}(9) & 88.2(4) \\ \mathrm{Hf}(1) & \mathrm{C}(101) & \mathrm{C}(10) & 87.3(4) & \mathrm{Hf}(1) & \mathrm{C}(101) & \mathrm{C}(11) & 91.9(6) \\ \mathrm{Hf}(1) & \mathrm{C}(101) & \mathrm{C}(12) & 91.9(4) & \mathrm{Hf}(1) & \mathrm{C}(101) & \mathrm{C}(13) & 90.9(5) \\ \mathrm{C}(9) & \mathrm{C}(101) & \mathrm{C}(10) & 71.9(6) & \mathrm{C}(9) & \mathrm{C}(101) & \mathrm{C}(11) & 144.3(9) \\ \mathrm{C}(9) & \mathrm{C}(101) & \mathrm{C}(12) & 144.3(7) & \mathrm{C}(9) & \mathrm{C}(101) & \mathrm{C}(13) & 73.7(7) \\ \mathrm{C}(10) & \mathrm{C}(101) & \mathrm{C}(11) & 72.5(8) & \mathrm{C}(10) & \mathrm{C}(101) & \mathrm{C}(12) & 143.8(7) \\ \mathrm{C}(10) & \mathrm{C}(101) & \mathrm{C}(13) & 145.6(7) & \mathrm{C}(11) & \mathrm{C}(101) & \mathrm{C}(12) & 71.3(9) \\ \mathrm{C}(11) & \mathrm{C}(101) & \mathrm{C}(13) & 141.9(9) & \mathrm{C}(12) & \mathrm{C}(101) & \mathrm{C}(13) & 70.6(7)\end{array}$


Table 8. Crystal Data for $\mathrm{CpCp} * \mathrm{Hf}\left(\mathrm{SnPh}_{3}\right) \mathrm{Cl}$ (4)

Empirical Formula

Formula Weight

Crystal Color, Habit

Crystal Dimensions

Crystal System

Lattice Type

No. of Reflections Used for Unit

Cell Determination (2 $\theta$ range)

Lattice Parameters
$\mathrm{C}_{33} \mathrm{H}_{35} \mathrm{SnClHf}$

764.27

yellow, block

$0.18 \times 0.20 \times 0.26 \mathrm{~mm}$

triclinic

Primitive

$5295(3.0-50.90)$

$\mathrm{a}=10.8007 \AA$

$\mathrm{b}=11.1272 \AA$

$c=13.5067 \AA$

$\alpha=97.2610^{\circ}$

$\beta=96.7350^{\circ}$

$\gamma=113.4230^{\circ}$

$\mathrm{V}=1452.3081 \AA^{3}$

$\mathrm{P} \overline{1}(\# 2)$

2

$1.748 \mathrm{~g} / \mathrm{cm}^{3}$

744.00

$45.36 \mathrm{~cm}^{-1}$

Table 9. Intensity Measurements for $\mathrm{CpCp} * \mathrm{Hf}\left(\mathrm{SnPh}_{3}\right) \mathrm{Cl}$ (4)

Diffractometer

Radiation

Crystal to Detector Distance

Temperature

Scan Type

Scan Rate

$2 \theta_{\text {max }}$

No. of Reflections Measured

Corrections
SMART

$\operatorname{MoK} \alpha(1=0.71069 \AA)$

graphite monochromated $60.0 \mathrm{~mm}$

$-149^{\circ} \mathrm{C}$

$\omega\left(0.3^{\circ}\right.$ per frame $)$

10.0 seconds per frame

50.90

Total: 7398

Unique: $4644\left(\mathrm{R}_{\mathrm{int}}=0.053\right)$

Lorentz-polarization Absorption

$(\operatorname{Tmax}=0.651, \operatorname{Tmin}=0.386)$ 
Table 10. Structure Solution and Refinement for $\mathrm{CpCp} * \mathrm{Hf}\left(\mathrm{SnPh}_{3}\right) \mathrm{Cl}$ (4)

$\begin{array}{ll}\text { Structure Solution } & \text { Direct Methods (SIR92) } \\ \text { Refinement } & \text { Full-matrix least-squares } \\ \text { Function Minimized } & \Sigma \omega(|\mathrm{Fol}-| \mathrm{Fcl})^{2} \\ \text { Least Squares Weights } & 1 / \mathrm{\sigma}^{2}(\mathrm{Fo})=4 \mathrm{Fo}^{2} / \mathrm{\sigma}^{2}\left(\mathrm{Fo}^{2}\right) \\ \text { p-factor } & 0.030 \\ \text { Anomalous Dispersion } & \text { All non-hydrogen atoms } \\ \text { No. Observations }(\mathrm{I}>3.00 \sigma(\mathrm{I})) & 3974 \\ \text { No. Variables } & 325 \\ \text { Reflection/Parameter Ratio } & 12.23 \\ \text { Residuals: R; Rw; Rall } & 0.032 ; 0.037 ; 0.038 \\ \text { Goodness of Fit Indicator } & 1.41 \\ \text { Max Shift/Error in Final Cycle } & 0.00 \\ \text { Maximum peak in Final Diff. Map } & 1.67 \mathrm{e}^{-} / \AA^{3} \\ \text { Minimum peak in Final Diff. Map } & -1.72 \mathrm{e}^{-} / \AA^{3}\end{array}$

Table 11. Atomic coordinates and $\mathrm{B}_{\text {iso }} / \mathrm{B}_{\text {eq }}$ for $\mathrm{CpCp} * \mathrm{Hf}\left(\mathrm{SnPh}_{3}\right) \mathrm{Cl}$ (4)

$\begin{array}{lccll}\text { atom } & \mathrm{x} & \mathrm{y} & \mathrm{z} & \mathrm{B}_{\mathrm{eq}} \\ \mathrm{Hf}(1) & 0.24183(2) & -0.11652(2) & 0.27054(2) & 1.538(6) \\ \mathrm{Sn}(1) & -0.05140(4) & -0.17519(4) & 0.20511(3) & 1.423(8) \\ \mathrm{Cl}(1) & 0.2166(2) & -0.0512(2) & 0.4414(1) & 2.72(3) \\ \mathrm{C}(1) & 0.2509(9) & 0.0366(9) & 0.1477(6) & 4.2(2) \\ \mathrm{C}(2) & 0.2695(8) & 0.1101(7) & 0.2400(6) & 3.7(2) \\ \mathrm{C}(3) & 0.3848(9) & 0.1278(8) & 0.2969(7) & 4.7(2) \\ \mathrm{C}(4) & 0.4479(8) & 0.0660(10) & 0.246(1) & 5.0(3) \\ \mathrm{C}(5) & 0.361(2) & -0.0006(10) & 0.142(1) & 6.3(4) \\ \mathrm{C}(6) & 0.1451(6) & -0.3650(5) & 0.2092(5) & 1.8(1) \\ \mathrm{C}(7) & 0.1825(6) & -0.3427(6) & 0.3161(5) & 1.9(1) \\ \mathrm{C}(8) & 0.3273(6) & -0.2711(6) & 0.3444(5) & 2.4(1) \\ \mathrm{C}(9) & 0.3804(6) & -0.2473(6) & 0.2523(5) & 1.9(1) \\ \mathrm{C}(10) & 0.2688(6) & -0.3056(6) & 0.1697(5) & 2.0(1) \\ \mathrm{C}(11) & 0.0081(6) & -0.4567(6) & 0.1459(5) & 2.3(1) \\ \mathrm{C}(12) & 0.2772(7) & -0.3233(7) & 0.0586(5) & 2.7(2) \\ \mathrm{C}(13) & 0.5319(7) & -0.1924(7) & 0.2474(6) & 3.0(2) \\ \mathrm{C}(14) & 0.4140(7) & -0.2306(7) & 0.4491(5) & 3.0(2) \\ \mathrm{C}(15) & 0.0889(6) & -0.3977(6) & 0.3894(5) & 2.3(1) \\ \mathrm{C}(16) & -0.1886(5) & -0.3032(6) & 0.2926(5) & 1.7(1) \\ \mathrm{C}(17) & -0.1841(6) & -0.2516(7) & 0.3941(5) & 2.1(1) \\ \mathrm{C}(18) & -0.2644(7) & -0.3295(8) & 0.4541(5) & 3.2(2) \\ \mathrm{C}(19) & -0.3489(7) & -0.4611(8) & 0.4150(6) & 3.2(2) \\ \mathrm{C}(20) & -0.3581(6) & -0.5173(7) & 0.3156(6) & 2.7(2) \\ \mathrm{C}(21) & -0.2765(6) & -0.4376(6) & 0.2550(5) & 2.1(1) \\ \mathrm{C}(22) & -0.1602(6) & -0.2612(5) & 0.0498(4) & 1.7(1) \\ & & & \mathrm{S} 13 & \end{array}$




\begin{tabular}{|c|c|c|c|c|}
\hline $\mathrm{C}(23)$ & $-0.3039(6)$ & $-0.3242(6)$ & $0.0261(5)$ & 2.1(1) \\
\hline $\mathrm{C}(24)$ & $-0.3725(6)$ & $-0.3839(6)$ & $-0.0725(5)$ & $2.5(1)$ \\
\hline$C(25)$ & $-0.3023(6)$ & $-0.3815(6)$ & $-0.1507(5)$ & $2.5(1)$ \\
\hline$C(26)$ & $-0.1606(6)$ & $-0.3184(6)$ & $-0.1305(4)$ & $2.2(1)$ \\
\hline $\mathrm{C}(27)$ & $-0.0908(6)$ & $-0.2593(6)$ & $-0.0320(5)$ & $2.0(1)$ \\
\hline $\mathrm{C}(28)$ & $-0.0949(5)$ & $0.0003(6)$ & $0.2321(4)$ & $1.6(1)$ \\
\hline C(29) & $-0.1763(6)$ & $0.0273(6)$ & $0.1584(5)$ & $2.3(1)$ \\
\hline$C(30)$ & $-0.2069(6)$ & $0.1379(7)$ & $0.1775(5)$ & $2.8(1)$ \\
\hline $\mathrm{C}(31)$ & $-0.1567(7)$ & $0.2231(7)$ & $0.2698(6)$ & $2.7(2)$ \\
\hline$C(32)$ & $-0.0746(8)$ & $0.1995(7)$ & $0.3451(5)$ & $3.1(2)$ \\
\hline $\mathrm{C}(33)$ & $-0.0449(7)$ & $0.0886(6)$ & $0.3257(5)$ & $2.4(1)$ \\
\hline$C(100)$ & 0.3427 & 0.0680 & 0.2146 & 0.2000 \\
\hline $\mathrm{C}(101)$ & 0.2608 & -0.3063 & 0.2583 & 0.2000 \\
\hline $\mathrm{H}(1)$ & 0.1807 & 0.0193 & 0.0904 & 5.0234 \\
\hline $\mathrm{H}(2)$ & 0.2091 & 0.1493 & 0.2582 & 4.4662 \\
\hline $\mathrm{H}(3)$ & 0.4221 & 0.1861 & 0.3637 & 5.6566 \\
\hline $\mathrm{H}(4)$ & 0.5382 & 0.0696 & 0.2656 & 6.0049 \\
\hline $\mathrm{H}(5)$ & 0.3932 & -0.0383 & 0.0880 & 7.5968 \\
\hline $\mathrm{H}(6)$ & 0.0146 & -0.5322 & 0.1089 & 2.7119 \\
\hline $\mathrm{H}(7)$ & -0.0583 & -0.4847 & 0.1873 & 2.7119 \\
\hline $\mathrm{H}(8)$ & -0.0185 & -0.4105 & 0.0981 & 2.7119 \\
\hline $\mathrm{H}(9)$ & 0.0945 & -0.3284 & 0.4409 & 2.7528 \\
\hline $\mathrm{H}(10)$ & 0.1115 & -0.4608 & 0.4200 & 2.7528 \\
\hline $\mathrm{H}(11)$ & -0.0056 & -0.4435 & 0.3540 & 2.7528 \\
\hline $\mathrm{H}(12)$ & 0.3548 & -0.2558 & 0.4977 & 3.6307 \\
\hline $\mathrm{H}(13)$ & 0.4734 & -0.2728 & 0.4521 & 3.6307 \\
\hline $\mathrm{H}(14)$ & 0.4646 & -0.1361 & 0.4644 & 3.6307 \\
\hline $\mathrm{H}(15)$ & 0.5705 & -0.2483 & 0.2708 & 3.6341 \\
\hline $\mathrm{H}(16)$ & 0.5780 & -0.1049 & 0.2903 & 3.6341 \\
\hline $\mathrm{H}(17)$ & 0.5451 & -0.1832 & 0.1800 & 3.6341 \\
\hline $\mathrm{H}(18)$ & 0.3257 & -0.3752 & 0.0442 & 3.2315 \\
\hline $\mathrm{H}(19)$ & 0.3245 & -0.2368 & 0.0415 & 3.2315 \\
\hline $\mathrm{H}(20)$ & 0.1874 & -0.3639 & 0.0188 & 3.2315 \\
\hline $\mathrm{H}(21)$ & -0.1236 & -0.1606 & 0.4221 & 2.5699 \\
\hline $\mathrm{H}(22)$ & -0.2633 & -0.2923 & 0.5224 & 3.8198 \\
\hline $\mathrm{H}(23)$ & -0.4023 & -0.5145 & 0.4583 & 3.8504 \\
\hline $\mathrm{H}(24)$ & -0.4217 & -0.6081 & 0.2904 & 3.2827 \\
\hline $\mathrm{H}(25)$ & -0.2787 & -0.4764 & 0.1854 & 2.4678 \\
\hline $\mathrm{H}(26)$ & -0.3560 & -0.3238 & 0.0787 & 2.5457 \\
\hline $\mathrm{H}(27)$ & -0.4708 & -0.4270 & -0.0849 & 2.9663 \\
\hline $\mathrm{H}(28)$ & -0.3505 & -0.4233 & -0.2194 & 3.0368 \\
\hline H(29) & -0.1095 & -0.3154 & -0.1847 & 2.6461 \\
\hline $\mathrm{H}(30)$ & 0.0078 & -0.2168 & -0.0202 & 2.3810 \\
\hline $\mathrm{H}(31)$ & -0.2118 & -0.0316 & 0.0924 & 2.7446 \\
\hline $\mathrm{H}(32)$ & -0.2636 & 0.1560 & 0.1260 & 3.3431 \\
\hline $\mathrm{H}(33)$ & -0.1824 & 0.2968 & 0.2826 & 3.2347 \\
\hline
\end{tabular}




$\begin{array}{lrrrr}\mathrm{H}(34) & -0.0398 & 0.2597 & 0.4104 & 3.7415 \\ \mathrm{H}(35) & 0.0120 & 0.0721 & 0.3784 & 2.8367\end{array}$

$B_{\text {eq }}=8 / 3 \pi^{2}\left(U_{11}\left(a a^{*}\right)^{2}+U_{22}\left(b b^{*}\right)^{2}+U_{33}\left(c c^{*}\right)^{2}+2 U_{12}\left(a a^{*} b b^{*}\right) \cos \gamma+2 U_{13}\left(a a^{*} c c^{*}\right) \cos \beta+\right.$ $\left.2 \mathrm{U}_{23}(\mathrm{bb} * \mathrm{cc} *) \cos \alpha\right)$

Table 12. Anisotropic Displacement Parameters for $\mathrm{CpCp} * \mathrm{Hf}\left(\mathrm{SnPh}_{3}\right) \mathrm{Cl}(4)$

\begin{tabular}{|c|c|c|c|c|c|c|}
\hline atom & $\mathrm{U}_{11}$ & $\mathrm{U}_{22}$ & $\mathrm{U}_{33}$ & $\mathrm{U}_{12}$ & $\mathrm{U}_{13}$ & $\mathrm{U}_{23}$ \\
\hline $\mathrm{Hf}(1)$ & $0.0176(1)$ & $0.0191(1)$ & $0.0209(2)$ & $0.00705(10)$ & $0.00135(9)$ & $0.00479(10)$ \\
\hline $\operatorname{Sn}(1)$ & $0.0180(2)$ & $0.0183(2)$ & $0.0172(2)$ & $0.0082(2)$ & $0.0019(2)$ & $0.0015(2)$ \\
\hline $\mathrm{Cl}(1)$ & $0.0425(9)$ & $0.0317(8)$ & $0.0229(8)$ & $0.0151(7)$ & $0.0009(7)$ & $-0.0015(7)$ \\
\hline $\mathrm{C}(1)$ & $0.074(6)$ & $0.046(5)$ & $0.032(5)$ & $-0.008(4)$ & $-0.013(4)$ & $0.032(4)$ \\
\hline$C(2)$ & $0.057(5)$ & $0.036(4)$ & $0.061(6)$ & $0.021(4)$ & $0.020(4)$ & $0.032(4)$ \\
\hline $\mathrm{C}(3)$ & $0.065(6)$ & $0.028(4)$ & $0.070(6)$ & $0.003(4)$ & $-0.004(5)$ & $0.013(4)$ \\
\hline $\mathrm{C}(4)$ & $0.036(4)$ & $0.057(6)$ & $0.14(1)$ & $0.022(4)$ & $0.037(6)$ & $0.057(7)$ \\
\hline $\mathrm{C}(5)$ & $0.25(2)$ & $0.041(5)$ & $0.13(1)$ & $0.080(8)$ & $0.18(1)$ & $0.061(7)$ \\
\hline$C(6)$ & $0.025(3)$ & $0.016(3)$ & $0.032(4)$ & $0.013(3)$ & $0.007(3)$ & $0.009(3)$ \\
\hline$C(7)$ & $0.025(3)$ & $0.017(3)$ & $0.034(4)$ & $0.013(2)$ & $0.008(3)$ & $0.007(3)$ \\
\hline $\mathrm{C}(8)$ & $0.029(3)$ & $0.030(3)$ & $0.035(4)$ & $0.018(3)$ & $0.001(3)$ & $0.009(3)$ \\
\hline$C(9)$ & $0.023(3)$ & $0.029(3)$ & $0.031(4)$ & $0.015(3)$ & $0.006(3)$ & $0.010(3)$ \\
\hline$C(10)$ & $0.033(3)$ & $0.028(3)$ & $0.025(3)$ & $0.019(3)$ & $0.007(3)$ & $0.004(3)$ \\
\hline $\mathrm{C}(11)$ & $0.032(3)$ & $0.022(3)$ & $0.031(4)$ & $0.014(3)$ & $0.001(3)$ & $-0.001(3)$ \\
\hline$C(12)$ & $0.044(4)$ & $0.038(4)$ & $0.031(4)$ & $0.023(3)$ & $0.015(3)$ & $0.005(3)$ \\
\hline $\mathrm{C}(13)$ & $0.025(3)$ & $0.046(4)$ & $0.059(5)$ & $0.020(3)$ & $0.013(3)$ & $0.014(4)$ \\
\hline$C(14)$ & $0.035(4)$ & $0.041(4)$ & $0.033(4)$ & $0.012(3)$ & $-0.007(3)$ & $0.010(3)$ \\
\hline$C(15)$ & $0.034(3)$ & $0.024(3)$ & $0.032(4)$ & $0.013(3)$ & $0.011(3)$ & $0.009(3)$ \\
\hline$C(16)$ & $0.017(3)$ & $0.027(3)$ & $0.025(3)$ & $0.012(2)$ & $0.005(2)$ & $0.010(3)$ \\
\hline$C(17)$ & $0.027(3)$ & $0.037(4)$ & $0.029(4)$ & $0.017(3)$ & $0.004(3)$ & $0.008(3)$ \\
\hline$C(18)$ & $0.046(4)$ & $0.051(5)$ & $0.030(4)$ & $0.025(4)$ & $0.018(3)$ & $0.018(3)$ \\
\hline$C(19)$ & $0.045(4)$ & $0.053(5)$ & $0.060(5)$ & $0.025(4)$ & $0.030(4)$ & $0.043(4)$ \\
\hline$C(20)$ & $0.030(3)$ & $0.030(4)$ & $0.057(5)$ & $0.015(3)$ & $0.011(3)$ & $0.018(3)$ \\
\hline$C(21)$ & $0.020(3)$ & $0.028(3)$ & $0.032(4)$ & $0.012(3)$ & $0.004(3)$ & $0.007(3)$ \\
\hline$C(22)$ & $0.026(3)$ & $0.018(3)$ & $0.021(3)$ & $0.014(2)$ & $0.003(2)$ & $0.002(2)$ \\
\hline$C(23)$ & $0.028(3)$ & $0.031(3)$ & $0.023(3)$ & $0.015(3)$ & $0.007(3)$ & $0.004(3)$ \\
\hline$C(24)$ & $0.023(3)$ & $0.029(3)$ & $0.031(4)$ & $0.007(3)$ & $-0.005(3)$ & $0.004(3)$ \\
\hline$C(25)$ & $0.036(4)$ & $0.029(3)$ & $0.019(3)$ & $0.011(3)$ & $-0.004(3)$ & $0.002(3)$ \\
\hline$C(26)$ & $0.040(4)$ & $0.032(3)$ & $0.016(3)$ & $0.023(3)$ & $0.005(3)$ & $0.003(3)$ \\
\hline$C(27)$ & $0.023(3)$ & $0.029(3)$ & $0.030(4)$ & $0.015(3)$ & $0.006(3)$ & $0.016(3)$ \\
\hline $\mathrm{C}(28)$ & $0.021(3)$ & $0.021(3)$ & $0.019(3)$ & $0.006(2)$ & $0.006(2)$ & $0.005(2)$ \\
\hline$C(29)$ & $0.022(3)$ & $0.025(3)$ & $0.028(3)$ & $0.007(3)$ & $0.008(3)$ & $0.004(3)$ \\
\hline$C(30)$ & $0.027(3)$ & $0.037(4)$ & $0.042(4)$ & $0.017(3)$ & $0.007(3)$ & $0.017(3)$ \\
\hline $\mathrm{C}(31)$ & $0.041(4)$ & $0.029(3)$ & $0.047(5)$ & $0.019(3)$ & $0.024(3)$ & $0.009(3)$ \\
\hline$C(32)$ & $0.058(4)$ & $0.035(4)$ & $0.031(4)$ & $0.021(4)$ & $0.018(3)$ & $0.001(3)$ \\
\hline$C(33)$ & $0.042(4)$ & $0.029(3)$ & $0.022(3)$ & $0.019(3)$ & $0.008(3)$ & $0.006(3)$ \\
\hline
\end{tabular}


The general temperature factor expression:

$\exp \left(-2 \pi^{2}\left(a^{*}{ }^{2} U_{11} h^{2}+b * 2 U_{22} k^{2}+c * 2 U_{33} l^{2}+2 a * b * U_{12} h k+2 a * c * U_{13} h l+2 b * c * U_{23} k l\right)\right)$

Table 13. Bond Lengths $(\AA)$ for $\mathrm{CpCp} * \mathrm{Hf}\left(\mathrm{SnPh}_{3}\right) \mathrm{Cl}(\mathbf{4})$

$\begin{array}{llllll}\text { atom } & \text { atom } & \text { distance } & \text { atom } & \text { atom } & \text { distance } \\ \mathrm{Hf}(1) & \mathrm{Sn}(1) & 2.9650(4) & \mathrm{Hf}(1) & \mathrm{Cl}(1) & 2.411(2) \\ \mathrm{Hf}(1) & \mathrm{C}(1) & 2.507(7) & \mathrm{Hf}(1) & \mathrm{C}(2) & 2.512(7) \\ \mathrm{Hf}(1) & \mathrm{C}(3) & 2.488(8) & \mathrm{Hf}(1) & \mathrm{C}(4) & 2.435(7) \\ \mathrm{Hf}(1) & \mathrm{C}(5) & 2.466(7) & \mathrm{Hf}(1) & \mathrm{C}(6) & 2.519(6) \\ \mathrm{Hf}(1) & \mathrm{C}(7) & 2.515(5) & \mathrm{Hf}(1) & \mathrm{C}(8) & 2.507(6) \\ \mathrm{Hf}(1) & \mathrm{C}(9) & 2.479(6) & \mathrm{Hf}(1) & \mathrm{C}(10) & 2.495(6) \\ \mathrm{Hf}(1) & \mathrm{C}(100) & 2.1864(2) & \mathrm{Hf}(1) & \mathrm{C}(101) & 2.1891(2) \\ \mathrm{Sn}(1) & \mathrm{C}(16) & 2.186(5) & \mathrm{Sn}(1) & \mathrm{C}(22) & 2.172(6) \\ \mathrm{Sn}(1) & \mathrm{C}(28) & 2.181(6) & \mathrm{C}(1) & \mathrm{C}(2) & 1.35(1) \\ \mathrm{C}(1) & \mathrm{C}(5) & 1.41(2) & \mathrm{C}(1) & \mathrm{C}(100) & 1.164(8) \\ \mathrm{C}(2) & \mathrm{C}(3) & 1.31(1) & \mathrm{C}(2) & \mathrm{C}(100) & 1.131(8) \\ \mathrm{C}(3) & \mathrm{C}(4) & 1.33(1) & \mathrm{C}(3) & \mathrm{C}(100) & 1.157(10) \\ \mathrm{C}(4) & \mathrm{C}(5) & 1.51(2) & \mathrm{C}(4) & \mathrm{C}(100) & 1.175(8) \\ \mathrm{C}(5) & \mathrm{C}(100) & 1.244(9) & \mathrm{C}(6) & \mathrm{C}(7) & 1.416(9) \\ \mathrm{C}(6) & \mathrm{C}(10) & 1.435(8) & \mathrm{C}(6) & \mathrm{C}(11) & 1.498(8) \\ \mathrm{C}(6) & \mathrm{C}(101) & 1.215(6) & \mathrm{C}(7) & \mathrm{C}(8) & 1.421(8) \\ \mathrm{C}(7) & \mathrm{C}(15) & 1.507(8) & \mathrm{C}(7) & \mathrm{C}(101) & 1.205(6) \\ \mathrm{C}(8) & \mathrm{C}(9) & 1.441(9) & \mathrm{C}(8) & \mathrm{C}(14) & 1.507(9) \\ \mathrm{C}(8) & \mathrm{C}(101) & 1.220(7) & \mathrm{C}(9) & \mathrm{C}(10) & 1.415(9) \\ \mathrm{C}(9) & \mathrm{C}(13) & 1.515(8) & \mathrm{C}(9) & \mathrm{C}(101) & 1.213(5) \\ \mathrm{C}(10) & \mathrm{C}(12) & 1.506(9) & \mathrm{C}(10) & \mathrm{C}(101) & 1.211(6) \\ \mathrm{C}(16) & \mathrm{C}(17) & 1.405(9) & \mathrm{C}(16) & \mathrm{C}(21) & 1.400(9) \\ \mathrm{C}(17) & \mathrm{C}(18) & 1.380(9) & \mathrm{C}(18) & \mathrm{C}(19) & 1.37(1) \\ \mathrm{C}(19) & \mathrm{C}(20) & 1.38(1) & \mathrm{C}(20) & \mathrm{C}(21) & 1.401(9) \\ \mathrm{C}(22) & \mathrm{C}(23) & 1.402(8) & \mathrm{C}(22) & \mathrm{C}(27) & 1.403(8) \\ \mathrm{C}(23) & \mathrm{C}(24) & 1.384(9) & \mathrm{C}(24) & \mathrm{C}(25) & 1.368(9) \\ \mathrm{C}(25) & \mathrm{C}(26) & 1.382(9) & \mathrm{C}(26) & \mathrm{C}(27) & 1.385(9) \\ \mathrm{C}(28) & \mathrm{C}(29) & 1.391(8) & \mathrm{C}(28) & \mathrm{C}(33) & 1.401(9) \\ \mathrm{C}(29) & \mathrm{C}(30) & 1.398(9) & \mathrm{C}(30) & \mathrm{C}(31) & 1.37(1) \\ \mathrm{C}(31) & \mathrm{C}(32) & 1.39(1) & \mathrm{C}(33) & 1.395(9)\end{array}$

Table 14. Bond Angles $\left({ }^{\circ}\right)$ for $\mathrm{CpCp} * \mathrm{Hf}\left(\mathrm{SnPh}_{3}\right) \mathrm{Cl}(\mathbf{4})$

$\begin{array}{llllllll}\text { atom } & \text { atom } & \text { atom } & \text { angle } & \text { atom } & \text { atom } & \text { atom } & \text { angle } \\ \operatorname{Sn}(1) & \operatorname{Hf}(1) & \mathrm{Cl}(1) & 87.65(4) & \mathrm{Sn}(1) & \mathrm{Hf}(1) & \mathrm{C}(1) & 77.1(2) \\ \mathrm{Sn}(1) & \mathrm{Hf}(1) & \mathrm{C}(2) & 81.1(2) & \mathrm{Sn}(1) & \mathrm{Hf}(1) & \mathrm{C}(3) & 110.1(2) \\ \mathrm{Sn}(1) & \mathrm{Hf}(1) & \mathrm{C}(4) & 130.0(2) & \mathrm{Sn}(1) & \mathrm{Hf}(1) & \mathrm{C}(5) & 105.2(4) \\ \mathrm{Sn}(1) & \mathrm{Hf}(1) & \mathrm{C}(6) & 80.0(1) & \mathrm{Sn}(1) & \mathrm{Hf}(1) & \mathrm{C}(7) & 92.1(1) \\ \mathrm{Sn}(1) & \mathrm{Hf}(1) & \mathrm{C}(8) & 124.9(1) & \mathrm{Sn}(1) & \mathrm{Hf}(1) & \mathrm{C}(9) & 134.3(1) \\ \mathrm{Sn}(1) & \mathrm{Hf}(1) & \mathrm{C}(10) & 103.6(1) & \mathrm{Sn}(1) & \mathrm{Hf}(1) & \mathrm{C}(100) & 101.17(1)\end{array}$




\begin{tabular}{|c|c|c|c|c|c|c|c|}
\hline $\operatorname{Sn}(1)$ & $\operatorname{Hf}(1)$ & $C(101)$ & $108.12(1)$ & $\mathrm{Cl}(1)$ & $\operatorname{Hf}(1)$ & $C(1)$ & $118.5(3)$ \\
\hline $\mathrm{Cl}(1)$ & $\operatorname{Hf}(1)$ & $C(2)$ & $88.1(2)$ & $\mathrm{Cl}(1)$ & $\operatorname{Hf}(1)$ & $\mathrm{C}(3)$ & $80.5(2)$ \\
\hline $\mathrm{Cl}(1)$ & $\mathrm{Hf}(1)$ & $C(4)$ & $104.6(3)$ & $\mathrm{Cl}(1)$ & $\mathrm{Hf}(1)$ & $C(5)$ & $135.8(3)$ \\
\hline $\mathrm{Cl}(1)$ & $\mathrm{Hf}(1)$ & $C(6)$ & $114.3(1)$ & $\mathrm{Cl}(1)$ & $\mathrm{Hf}(1)$ & $C(7)$ & $84.8(1)$ \\
\hline $\mathrm{Cl}(1)$ & $\operatorname{Hf}(1)$ & $\mathrm{C}(8)$ & $84.9(2)$ & $\mathrm{Cl}(1)$ & $\operatorname{Hf}(1)$ & $\mathrm{C}(9)$ & $115.7(1)$ \\
\hline $\mathrm{Cl}(1)$ & $\operatorname{Hf}(1)$ & $\mathrm{C}(10)$ & $137.7(1)$ & $\mathrm{Cl}(1)$ & $\operatorname{Hf}(1)$ & $\mathrm{C}(100)$ & $106.41(4)$ \\
\hline $\mathrm{Cl}(1)$ & $\operatorname{Hf}(1)$ & $\mathrm{C}(101)$ & $108.68(4)$ & $\mathrm{C}(1)$ & $\operatorname{Hf}(1)$ & $C(2)$ & $31.2(3)$ \\
\hline $\mathrm{C}(1)$ & $\operatorname{Hf}(1)$ & $\mathrm{C}(3)$ & $52.1(3)$ & $\mathrm{C}(1)$ & $\operatorname{Hf}(1)$ & $\mathrm{C}(4)$ & $54.2(3)$ \\
\hline$C(1)$ & $\operatorname{Hf}(1)$ & $C(5)$ & $32.9(4)$ & $\mathrm{C}(1)$ & $\operatorname{Hf}(1)$ & $C(6)$ & $120.6(3)$ \\
\hline$C(1)$ & $\operatorname{Hf}(1)$ & $C(7)$ & $153.3(3)$ & $\mathrm{C}(1)$ & $\operatorname{Hf}(1)$ & $\mathrm{C}(8)$ & $150.3(3)$ \\
\hline$C(1)$ & $\operatorname{Hf}(1)$ & $\mathrm{C}(9)$ & $117.2(3)$ & $\mathrm{C}(1)$ & $\operatorname{Hf}(1)$ & $C(10)$ & $103.8(3)$ \\
\hline $\mathrm{C}(1)$ & $\operatorname{Hf}(1)$ & $\mathrm{C}(100)$ & $27.7(2)$ & $\mathrm{C}(1)$ & $\operatorname{Hf}(1)$ & $\mathrm{C}(101)$ & $132.8(3)$ \\
\hline$C(2)$ & $\operatorname{Hf}(1)$ & $\mathrm{C}(3)$ & $30.4(3)$ & $\mathrm{C}(2)$ & $\operatorname{Hf}(1)$ & $\mathrm{C}(4)$ & $51.8(3)$ \\
\hline$C(2)$ & $\operatorname{Hf}(1)$ & $C(5)$ & $53.9(3)$ & $\mathrm{C}(2)$ & $\operatorname{Hf}(1)$ & $C(6)$ & $149.8(2)$ \\
\hline$C(2)$ & $\mathrm{Hf}(1)$ & $C(7)$ & $170.4(2)$ & $\mathrm{C}(2)$ & $\mathrm{Hf}(1)$ & $\mathrm{C}(8)$ & $152.6(2)$ \\
\hline$C(2)$ & $\operatorname{Hf}(1)$ & $\mathrm{C}(9)$ & $134.5(2)$ & $C(2)$ & $\operatorname{Hf}(1)$ & $C(10)$ & $133.5(2)$ \\
\hline$C(2)$ & $\operatorname{Hf}(1)$ & $\mathrm{C}(100)$ & $26.7(2)$ & $\mathrm{C}(2)$ & $\operatorname{Hf}(1)$ & $\mathrm{C}(101)$ & $160.7(2)$ \\
\hline $\mathrm{C}(3)$ & $\operatorname{Hf}(1)$ & $\mathrm{C}(4)$ & $31.3(3)$ & $\mathrm{C}(3)$ & $\operatorname{Hf}(1)$ & $\mathrm{C}(5)$ & $55.3(4)$ \\
\hline $\mathrm{C}(3)$ & $\operatorname{Hf}(1)$ & $C(6)$ & $163.1(3)$ & $\mathrm{C}(3)$ & $\operatorname{Hf}(1)$ & $C(7)$ & $152.6(3)$ \\
\hline$C(3)$ & $\operatorname{Hf}(1)$ & $\mathrm{C}(8)$ & $122.2(3)$ & $\mathrm{C}(3)$ & $\operatorname{Hf}(1)$ & $\mathrm{C}(9)$ & $112.1(3)$ \\
\hline $\mathrm{C}(3)$ & $\operatorname{Hf}(1)$ & $\mathrm{C}(10)$ & $129.9(3)$ & $\mathrm{C}(3)$ & $\operatorname{Hf}(1)$ & $\mathrm{C}(100)$ & $27.7(2)$ \\
\hline$C(3)$ & $\operatorname{Hf}(1)$ & $\mathrm{C}(101)$ & $141.0(2)$ & $\mathrm{C}(4)$ & $\operatorname{Hf}(1)$ & $\mathrm{C}(5)$ & $35.9(4)$ \\
\hline$C(4)$ & $\operatorname{Hf}(1)$ & $C(6)$ & $132.0(3)$ & $\mathrm{C}(4)$ & $\operatorname{Hf}(1)$ & $C(7)$ & $136.5(2)$ \\
\hline$C(4)$ & $\operatorname{Hf}(1)$ & $\mathrm{C}(8)$ & $104.6(2)$ & $\mathrm{C}(4)$ & $\operatorname{Hf}(1)$ & $\mathrm{C}(9)$ & $83.7(3)$ \\
\hline $\mathrm{C}(4)$ & $\operatorname{Hf}(1)$ & $\mathrm{C}(10)$ & $98.8(3)$ & $\mathrm{C}(4)$ & $\operatorname{Hf}(1)$ & $\mathrm{C}(100)$ & $28.8(2)$ \\
\hline$C(4)$ & $\operatorname{Hf}(1)$ & $\mathrm{C}(101)$ & $112.9(2)$ & $\mathrm{C}(5)$ & $\operatorname{Hf}(1)$ & $\mathrm{C}(6)$ & $109.6(3)$ \\
\hline$C(5)$ & $\operatorname{Hf}(1)$ & $\mathrm{C}(7)$ & $135.2(2)$ & $\mathrm{C}(5)$ & $\operatorname{Hf}(1)$ & $\mathrm{C}(8)$ & $117.6(4)$ \\
\hline $\mathrm{C}(5)$ & $\operatorname{Hf}(1)$ & $\mathrm{C}(9)$ & $85.3(3)$ & $C(5)$ & $\operatorname{Hf}(1)$ & $C(10)$ & $80.9(2)$ \\
\hline$C(5)$ & $\operatorname{Hf}(1)$ & $\mathrm{C}(100)$ & $30.3(2)$ & $C(5)$ & $\operatorname{Hf}(1)$ & $\mathrm{C}(101)$ & $106.9(2)$ \\
\hline$C(6)$ & $\operatorname{Hf}(1)$ & $C(7)$ & $32.7(2)$ & $\mathrm{C}(6)$ & $\operatorname{Hf}(1)$ & $\mathrm{C}(8)$ & $54.8(2)$ \\
\hline$C(6)$ & $\operatorname{Hf}(1)$ & $\mathrm{C}(9)$ & $55.0(2)$ & $\mathrm{C}(6)$ & $\operatorname{Hf}(1)$ & $C(10)$ & $33.3(2)$ \\
\hline$C(6)$ & $\operatorname{Hf}(1)$ & $\mathrm{C}(100)$ & $139.3(1)$ & $\mathrm{C}(6)$ & $\operatorname{Hf}(1)$ & $\mathrm{C}(101)$ & $28.8(1)$ \\
\hline$C(7)$ & $\operatorname{Hf}(1)$ & $\mathrm{C}(8)$ & $32.9(2)$ & $C(7)$ & $\operatorname{Hf}(1)$ & $\mathrm{C}(9)$ & $54.9(2)$ \\
\hline$C(7)$ & $\operatorname{Hf}(1)$ & $\mathrm{C}(10)$ & $54.6(2)$ & $C(7)$ & $\operatorname{Hf}(1)$ & $\mathrm{C}(100)$ & $162.8(1)$ \\
\hline$C(7)$ & $\operatorname{Hf}(1)$ & $\mathrm{C}(101)$ & $28.6(1)$ & $\mathrm{C}(8)$ & $\operatorname{Hf}(1)$ & $\mathrm{C}(9)$ & $33.6(2)$ \\
\hline $\mathrm{C}(8)$ & $\operatorname{Hf}(1)$ & $\mathrm{C}(10)$ & $55.1(2)$ & $\mathrm{C}(8)$ & $\operatorname{Hf}(1)$ & $\mathrm{C}(100)$ & $133.3(1)$ \\
\hline $\mathrm{C}(8)$ & $\mathrm{Hf}(1)$ & $\mathrm{C}(101)$ & $29.1(2)$ & $\mathrm{C}(9)$ & $\operatorname{Hf}(1)$ & $C(10)$ & $33.0(2)$ \\
\hline$C(9)$ & $\operatorname{Hf}(1)$ & $\mathrm{C}(100)$ & $108.0(1)$ & $\mathrm{C}(9)$ & $\operatorname{Hf}(1)$ & $\mathrm{C}(101)$ & $29.3(1)$ \\
\hline$C(10)$ & $\operatorname{Hf}(1)$ & $\mathrm{C}(100)$ & $110.9(1)$ & $\mathrm{C}(10)$ & $\operatorname{Hf}(1)$ & $\mathrm{C}(101)$ & $29.0(1)$ \\
\hline$C(100)$ & $\operatorname{Hf}(1)$ & $\mathrm{C}(101)$ & $134.59(1)$ & $\mathrm{Hf}(1)$ & $\operatorname{Sn}(1)$ & $C(16)$ & $113.9(1)$ \\
\hline $\mathrm{Hf}(1)$ & $\operatorname{Sn}(1)$ & $\mathrm{C}(22)$ & $123.0(1)$ & $\operatorname{Hf}(1)$ & $\operatorname{Sn}(1)$ & $\mathrm{C}(28)$ & $113.4(1)$ \\
\hline$C(16)$ & $\operatorname{Sn}(1)$ & $C(22)$ & $102.7(2)$ & $C(16)$ & $\operatorname{Sn}(1)$ & $\mathrm{C}(28)$ & $100.5(2)$ \\
\hline $\mathrm{C}(22)$ & $\operatorname{Sn}(1)$ & $\mathrm{C}(28)$ & $100.3(2)$ & $\operatorname{Hf}(1)$ & $\mathrm{C}(1)$ & $\mathrm{C}(2)$ & $74.6(4)$ \\
\hline $\mathrm{Hf}(1)$ & $\mathrm{C}(1)$ & $\mathrm{C}(5)$ & $72.0(4)$ & $\operatorname{Hf}(1)$ & $\mathrm{C}(1)$ & $\mathrm{C}(100)$ & $60.7(3)$ \\
\hline$C(2)$ & $C(1)$ & $C(5)$ & $109.8(9)$ & $\mathrm{C}(2)$ & $\mathrm{C}(1)$ & $\mathrm{C}(100)$ & $52.9(5)$ \\
\hline$C(5)$ & $\mathrm{C}(1)$ & $\mathrm{C}(100)$ & $56.9(7)$ & $\operatorname{Hf}(1)$ & $\mathrm{C}(2)$ & $\mathrm{C}(1)$ & $74.2(4)$ \\
\hline
\end{tabular}




\begin{tabular}{|c|c|c|c|c|c|c|c|}
\hline $\operatorname{Hf}(1)$ & $C(2)$ & $C(3)$ & $73.8(4)$ & $\operatorname{Hf}(1)$ & $C(2)$ & $C(100)$ & $60.4(3)$ \\
\hline $\mathrm{C}(1)$ & $C(2)$ & $\mathrm{C}(3)$ & 111.1(8) & $C(1)$ & $\mathrm{C}(2)$ & $C(100)$ & $55.2(6)$ \\
\hline $\mathrm{C}(3)$ & $C(2)$ & $C(100)$ & $56.0(6)$ & $\mathrm{Hf}(1)$ & $C(3)$ & $C(2)$ & $75.8(5)$ \\
\hline $\operatorname{Hf}(1)$ & $C(3)$ & $C(4)$ & $72.2(5)$ & $\operatorname{Hf}(1)$ & $\mathrm{C}(3)$ & $C(100)$ & $61.5(3)$ \\
\hline $\mathrm{C}(2)$ & $\mathrm{C}(3)$ & $\mathrm{C}(4)$ & $110.0(9)$ & $C(2)$ & $\mathrm{C}(3)$ & $C(100)$ & $54.1(5)$ \\
\hline $\mathrm{C}(4)$ & $C(3)$ & $C(100)$ & $55.9(7)$ & $\operatorname{Hf}(1)$ & $\mathrm{C}(4)$ & $\mathrm{C}(3)$ & $76.5(5)$ \\
\hline $\operatorname{Hf}(1)$ & $\mathrm{C}(4)$ & $C(5)$ & $73.2(5)$ & $\operatorname{Hf}(1)$ & $\mathrm{C}(4)$ & $C(100)$ & $63.7(3)$ \\
\hline $\mathrm{C}(3)$ & $C(4)$ & $C(5)$ & $108.0(8)$ & $C(3)$ & $C(4)$ & $C(100)$ & $54.6(5)$ \\
\hline $\mathrm{C}(5)$ & $\mathrm{C}(4)$ & $C(100)$ & $53.4(6)$ & $\operatorname{Hf}(1)$ & $C(5)$ & $C(1)$ & 75.1(4) \\
\hline $\operatorname{Hf}(1)$ & $C(5)$ & $\mathrm{C}(4)$ & 70.9(4) & $\mathrm{Hf}(1)$ & $\mathrm{C}(5)$ & $C(100)$ & $62.3(3)$ \\
\hline $\mathrm{C}(1)$ & $C(5)$ & $\mathrm{C}(4)$ & $101.0(7)$ & $C(1)$ & $\mathrm{C}(5)$ & $C(100)$ & $51.6(5)$ \\
\hline $\mathrm{C}(4)$ & $\mathrm{C}(5)$ & $C(100)$ & $49.3(6)$ & $\operatorname{Hf}(1)$ & $\mathrm{C}(6)$ & $C(7)$ & $73.5(3)$ \\
\hline $\mathrm{Hf}(1)$ & $C(6)$ & $C(10)$ & $72.5(3)$ & $\mathrm{Hf}(1)$ & $C(6)$ & $\mathrm{C}(11)$ & $128.9(4)$ \\
\hline $\mathrm{Hf}(1)$ & $C(6)$ & $C(101)$ & $60.4(2)$ & $C(7)$ & $C(6)$ & $C(10)$ & $107.5(5)$ \\
\hline$C(7)$ & $C(6)$ & $\mathrm{C}(11)$ & $126.8(5)$ & $C(7)$ & $C(6)$ & $C(101)$ & $53.9(3)$ \\
\hline $\mathrm{C}(10)$ & $C(6)$ & $C(11)$ & $124.6(6)$ & $C(10)$ & $\mathrm{C}(6)$ & $C(101)$ & $53.6(3)$ \\
\hline $\mathrm{C}(11)$ & $C(6)$ & $C(101)$ & $170.6(5)$ & $\operatorname{Hf}(1)$ & $\mathrm{C}(7)$ & $C(6)$ & $73.8(3)$ \\
\hline $\operatorname{Hf}(1)$ & $C(7)$ & $C(8)$ & $73.2(3)$ & $\operatorname{Hf}(1)$ & $\mathrm{C}(7)$ & $C(15)$ & $124.4(4)$ \\
\hline $\operatorname{Hf}(1)$ & $C(7)$ & $C(101)$ & $60.5(2)$ & $C(6)$ & $C(7)$ & $\mathrm{C}(8)$ & $109.1(5)$ \\
\hline$C(6)$ & $C(7)$ & $C(15)$ & $126.6(5)$ & $C(6)$ & $C(7)$ & $C(101)$ & $54.5(3)$ \\
\hline $\mathrm{C}(8)$ & $\mathrm{C}(7)$ & $C(15)$ & $123.9(6)$ & $C(8)$ & $\mathrm{C}(7)$ & $C(101)$ & $54.6(4)$ \\
\hline$C(15)$ & $C(7)$ & $C(101)$ & $175.0(5)$ & $\mathrm{Hf}(1)$ & $\mathrm{C}(8)$ & $C(7)$ & $73.9(3)$ \\
\hline $\operatorname{Hf}(1)$ & $\mathrm{C}(8)$ & $\mathrm{C}(9)$ & $72.1(3)$ & $\operatorname{Hf}(1)$ & $\mathrm{C}(8)$ & $C(14)$ & $121.3(4)$ \\
\hline $\operatorname{Hf}(1)$ & $C(8)$ & $C(101)$ & $60.8(2)$ & $C(7)$ & $\mathrm{C}(8)$ & $\mathrm{C}(9)$ & 107.1(6) \\
\hline$C(7)$ & $C(8)$ & $C(14)$ & 128.1(6) & $C(7)$ & $\mathrm{C}(8)$ & $C(101)$ & $53.6(4)$ \\
\hline $\mathrm{C}(9)$ & $\mathrm{C}(8)$ & $C(14)$ & $124.8(5)$ & $\mathrm{C}(9)$ & $\mathrm{C}(8)$ & $C(101)$ & $53.4(4)$ \\
\hline$C(14)$ & $C(8)$ & $C(101)$ & $177.2(6)$ & $\mathrm{Hf}(1)$ & $\mathrm{C}(9)$ & $\mathrm{C}(8)$ & $74.3(3)$ \\
\hline $\operatorname{Hf}(1)$ & $\mathrm{C}(9)$ & $C(10)$ & 74.1(3) & $\operatorname{Hf}(1)$ & $\mathrm{C}(9)$ & $\mathrm{C}(13)$ & $126.3(4)$ \\
\hline $\operatorname{Hf}(1)$ & $\mathrm{C}(9)$ & $C(101)$ & $62.0(2)$ & $C(8)$ & $\mathrm{C}(9)$ & $\mathrm{C}(10)$ & $108.1(5)$ \\
\hline $\mathrm{C}(8)$ & $\mathrm{C}(9)$ & $C(13)$ & $123.7(6)$ & $C(8)$ & $\mathrm{C}(9)$ & $C(101)$ & $53.9(3)$ \\
\hline$C(10)$ & $\mathrm{C}(9)$ & $C(13)$ & $127.2(6)$ & $C(10)$ & $\mathrm{C}(9)$ & $\mathrm{C}(101)$ & $54.2(3)$ \\
\hline $\mathrm{C}(13)$ & $\mathrm{C}(9)$ & $C(101)$ & $171.5(6)$ & $\operatorname{Hf}(1)$ & $\mathrm{C}(10)$ & $\mathrm{C}(6)$ & $74.3(3)$ \\
\hline $\operatorname{Hf}(1)$ & $C(10)$ & $\mathrm{C}(9)$ & $72.9(3)$ & $\operatorname{Hf}(1)$ & $\mathrm{C}(10)$ & $\mathrm{C}(12)$ & $126.1(4)$ \\
\hline $\operatorname{Hf}(1)$ & $C(10)$ & $C(101)$ & $61.3(3)$ & $C(6)$ & $C(10)$ & $\mathrm{C}(9)$ & $108.2(5)$ \\
\hline$C(6)$ & $C(10)$ & $C(12)$ & $124.5(6)$ & $\mathrm{C}(6)$ & $\mathrm{C}(10)$ & $C(101)$ & $53.8(3)$ \\
\hline $\mathrm{C}(9)$ & $C(10)$ & $C(12)$ & $126.6(5)$ & $C(9)$ & $\mathrm{C}(10)$ & $C(101)$ & $54.3(4)$ \\
\hline$C(12)$ & $C(10)$ & $C(101)$ & $172.4(6)$ & $\operatorname{Sn}(1)$ & $\mathrm{C}(16)$ & $\mathrm{C}(17)$ & $119.0(4)$ \\
\hline $\operatorname{Sn}(1)$ & $C(16)$ & $\mathrm{C}(21)$ & $123.6(4)$ & $C(17)$ & $\mathrm{C}(16)$ & $\mathrm{C}(21)$ & $117.3(5)$ \\
\hline$C(16)$ & $\mathrm{C}(17)$ & $C(18)$ & $121.5(6)$ & $C(17)$ & $\mathrm{C}(18)$ & $\mathrm{C}(19)$ & $119.7(7)$ \\
\hline $\mathrm{C}(18)$ & $C(19)$ & $C(20)$ & $121.4(6)$ & $C(19)$ & $\mathrm{C}(20)$ & $\mathrm{C}(21)$ & $118.6(6)$ \\
\hline$C(16)$ & $C(21)$ & $C(20)$ & $121.5(6)$ & $\operatorname{Sn}(1)$ & $C(22)$ & $\mathrm{C}(23)$ & $121.6(4)$ \\
\hline $\operatorname{Sn}(1)$ & $C(22)$ & $C(27)$ & $122.0(4)$ & $C(23)$ & $\mathrm{C}(22)$ & $\mathrm{C}(27)$ & $116.3(5)$ \\
\hline $\mathrm{C}(22)$ & $C(23)$ & $C(24)$ & $121.4(5)$ & $C(23)$ & $C(24)$ & $C(25)$ & $121.1(5)$ \\
\hline$C(24)$ & $C(25)$ & $C(26)$ & 119.2(6) & $C(25)$ & $C(26)$ & $\mathrm{C}(27)$ & $120.3(6)$ \\
\hline$C(22)$ & $C(27)$ & $C(26)$ & $121.8(5)$ & $\operatorname{Sn}(1)$ & $\mathrm{C}(28)$ & $\mathrm{C}(29)$ & $121.6(4)$ \\
\hline $\operatorname{Sn}(1)$ & $C(28)$ & $C(33)$ & 121.1(4) & $C(29)$ & $\mathrm{C}(28)$ & $\mathrm{C}(33)$ & $117.2(5)$ \\
\hline
\end{tabular}




$\begin{array}{llllllll}\mathrm{C}(28) & \mathrm{C}(29) & \mathrm{C}(30) & 121.2(6) & \mathrm{C}(29) & \mathrm{C}(30) & \mathrm{C}(31) & 120.6(6) \\ \mathrm{C}(30) & \mathrm{C}(31) & \mathrm{C}(32) & 119.8(6) & \mathrm{C}(31) & \mathrm{C}(32) & \mathrm{C}(33) & 119.3(6) \\ \mathrm{C}(28) & \mathrm{C}(33) & \mathrm{C}(32) & 121.9(6) & \mathrm{Hf}(1) & \mathrm{C}(100) & \mathrm{C}(1) & 91.7(3) \\ \mathrm{Hf}(1) & \mathrm{C}(100) & \mathrm{C}(2) & 92.9(3) & \mathrm{Hf}(1) & \mathrm{C}(100) & \mathrm{C}(3) & 90.8(4) \\ \mathrm{Hf}(1) & \mathrm{C}(100) & \mathrm{C}(4) & 87.4(4) & \mathrm{Hf}(1) & \mathrm{C}(100) & \mathrm{C}(5) & 87.4(3) \\ \mathrm{C}(1) & \mathrm{C}(100) & \mathrm{C}(2) & 71.9(7) & \mathrm{C}(1) & \mathrm{C}(100) & \mathrm{C}(3) & 141.8(7) \\ \mathrm{C}(1) & \mathrm{C}(100) & \mathrm{C}(4) & 148.7(8) & \mathrm{C}(1) & \mathrm{C}(100) & \mathrm{C}(5) & 71.4(9) \\ \mathrm{C}(2) & \mathrm{C}(100) & \mathrm{C}(3) & 69.9(6) & \mathrm{C}(2) & \mathrm{C}(100) & \mathrm{C}(4) & 139.4(8) \\ \mathrm{C}(2) & \mathrm{C}(100) & \mathrm{C}(5) & 143.3(10) & \mathrm{C}(3) & \mathrm{C}(100) & \mathrm{C}(4) & 69.5(8) \\ \mathrm{C}(3) & \mathrm{C}(100) & \mathrm{C}(5) & 146.7(10) & \mathrm{C}(4) & \mathrm{C}(100) & \mathrm{C}(5) & 77.2(9) \\ \mathrm{Hf}(1) & \mathrm{C}(101) & \mathrm{C}(6) & 90.8(3) & \mathrm{Hf}(1) & \mathrm{C}(101) & \mathrm{C}(7) & 90.9(3) \\ \mathrm{Hf}(1) & \mathrm{C}(101) & \mathrm{C}(8) & 90.1(3) & \mathrm{Hf}(1) & \mathrm{C}(101) & \mathrm{C}(9) & 88.7(3) \\ \mathrm{Hf}(1) & \mathrm{C}(101) & \mathrm{C}(10) & 89.6(3) & \mathrm{C}(6) & \mathrm{C}(101) & \mathrm{C}(7) & 71.6(4) \\ \mathrm{C}(6) & \mathrm{C}(101) & \mathrm{C}(8) & 143.4(4) & \mathrm{C}(6) & \mathrm{C}(101) & \mathrm{C}(9) & 144.0(4) \\ \mathrm{C}(6) & \mathrm{C}(101) & \mathrm{C}(10) & 72.6(4) & \mathrm{C}(7) & \mathrm{C}(101) & \mathrm{C}(8) & 71.8(4) \\ \mathrm{C}(7) & \mathrm{C}(101) & \mathrm{C}(9) & 144.4(4) & \mathrm{C}(7) & \mathrm{C}(101) & \mathrm{C}(10) & 144.2(4) \\ \mathrm{C}(8) & \mathrm{C}(101) & \mathrm{C}(9) & 72.6(4) & \mathrm{C}(8) & \mathrm{C}(101) & \mathrm{C}(10) & 144.1(4) \\ \mathrm{C}(9) & \mathrm{C}(101) & \mathrm{C}(10) & 71.4(4) & & & & \end{array}$

Table 15. Crystal Data for $\mathrm{CpCp} * \mathrm{Hf}\left(\mathrm{SnPh}_{3}\right) \mathrm{NMe}_{2} \bullet 0.75\left(\mathrm{C}_{4} \mathrm{H}_{10} \mathrm{O}\right)(\mathbf{8})$

\begin{tabular}{|c|c|}
\hline Empirical Formula & $\mathrm{C}_{38} \mathrm{H}_{51.50} \mathrm{NO}_{0.75} \mathrm{SnHf}$ \\
\hline Formula Weight & 831.51 \\
\hline Crystal Color, Habit & yellow, plates \\
\hline Crystal Dimensions & $0.21 \times 0.24 \times 0.07 \mathrm{~mm}$ \\
\hline Crystal System & triclinic \\
\hline Lattice Type & Primitive \\
\hline No. of Reflections Used for Unit & \\
\hline Cell Determination (2 $\theta$ range) & $6269\left(3.0-51.3^{\circ}\right)$ \\
\hline Lattice Parameters & $a=11.9538(6) \AA$ \\
\hline & $\mathrm{b}=13.5266(7) \AA$ \\
\hline & $c=21.671(1) \AA$ \\
\hline & $\alpha=88.331(1)^{\mathrm{O}}$ \\
\hline & $\beta=84.463(1)^{\mathrm{O}}$ \\
\hline & $\gamma=80.483(1)^{\mathrm{O}}$ \\
\hline & $\mathrm{V}=3439.5(3) \AA^{3}$ \\
\hline Space Group & $\mathrm{P} \overline{1}(\# 2)$ \\
\hline $\mathrm{Z}$ value & 4 \\
\hline $\mathrm{D}_{\text {calc }}$ & $1.606 \mathrm{~g} / \mathrm{cm}^{3}$ \\
\hline $\mathrm{F}_{000}$ & 1658.00 \\
\hline$\mu(\operatorname{MoK} \alpha)$ & $37.65 \mathrm{~cm}^{-1}$ \\
\hline
\end{tabular}


Table 16. Intensity Measurements for $\mathrm{CpCp} * \mathrm{Hf}\left(\mathrm{SnPh}_{3}\right) \mathrm{NMe}_{2} \bullet 0.75\left(\mathrm{C}_{4} \mathrm{H}_{10} \mathrm{O}\right)(\mathbf{8})$

Diffractometer

Radiation

Crystal to Detector Distance

Temperature

Scan Type

Scan Rate

$2 \theta_{\max }$

No. of Reflections Measured

Corrections
SMART

$\operatorname{MoK} \alpha(1=0.71069 \AA)$

graphite monochromated

$60.0 \mathrm{~mm}$

$-153.0^{\circ} \mathrm{C}$

$\omega\left(0.3^{\circ}\right.$ per frame $)$

10.0 seconds per frame

$51.3^{\circ}$

Total: 17608

Unique: $11122\left(\mathrm{R}_{\mathrm{int}}=0.055\right)$

Lorentz-polarization Absorption

$($ Tmax $=0.809$, Tmin $=0.387)$

Table 17. Structure Solution and Refinement for $\mathrm{CpCp} * \mathrm{Hf}\left(\mathrm{SnPh}_{3}\right) \mathrm{NMe}_{2} \bullet 0.75\left(\mathrm{C}_{4} \mathrm{H}_{10} \mathrm{O}\right)(\mathbf{8})$

Structure Solution

Refinement

Function Minimized

Least Squares Weights

p-factor

Anomalous Dispersion

No. Observations $(\mathrm{I}>2.50 \sigma(\mathrm{I}))$

No. Variables

Reflection/Parameter Ratio

Residuals: R; Rw; Rall

Goodness of Fit Indicator

Max Shift/Error in Final Cycle

Maximum peak in Final Diff. Map

Minimum peak in Final Diff. Map
Direct Methods (SIR92)

Full-matrix least-squares

$\Sigma \omega(|\mathrm{Fol}-| \mathrm{Fcl})^{2}$

$1 / \mathrm{\sigma}^{2}(\mathrm{Fo})=4 \mathrm{Fo}^{2} / \mathrm{\sigma}^{2}\left(\mathrm{Fo}^{2}\right)$

0.030

All non-hydrogen atoms

7333

692

10.60

$0.049 ; 0.054 ; 0.082$

1.23

0.00

$1.55 \mathrm{e}^{-} / \AA^{3}$

$-2.36 \mathrm{e}^{-} / \AA^{3}$

Table 18. Atomic coordinates and $\mathrm{B}_{\text {iso }} / \mathrm{B}_{\text {eq }}$ for $\mathrm{CpCp} * \mathrm{Hf}\left(\mathrm{SnPh}_{3}\right) \mathrm{NMe}_{2} \cdot 0.75\left(\mathrm{C}_{4} \mathrm{H}_{10} \mathrm{O}\right)(\mathbf{8})$

$\begin{array}{lllcl}\text { atom } & \mathrm{x} & \mathrm{y} & \mathrm{z} & \mathrm{B}_{\mathrm{eq}} \\ \mathrm{Hf}(1) & 0.49199(4) & -0.11136(3) & -0.31333(2) & 1.65(1) \\ \mathrm{Hf}(2) & 0.02742(4) & -0.38319(3) & 0.19515(2) & 1.39(1) \\ \mathrm{Sn}(1) & 0.39361(6) & -0.29912(5) & -0.31293(3) & 1.48(2) \\ \mathrm{Sn}(2) & 0.12113(6) & -0.19695(5) & 0.21182(3) & 1.34(2) \\ \mathrm{O}(1) & 0.6575(7) & -0.1919(6) & -0.0100(3) & 2.5(2) \\ \mathrm{O}(2) & 0.051(1) & 0.494(1) & -0.4971(8) & 8.3(4) \\ \mathrm{N}(1) & 0.3578(8) & -0.0468(6) & -0.2526(4) & 1.7(2) \\ \mathrm{N}(2) & 0.1263(8) & -0.4544(7) & 0.2617(5) & 2.2(2) \\ \mathrm{C}(1) & 0.6149(10) & -0.2403(8) & -0.2508(5) & 2.1(2) \\ & & & \mathrm{S} 20 & \end{array}$




\begin{tabular}{|c|c|c|c|c|}
\hline $\mathrm{C}(2)$ & $0.5738(10)$ & $-0.1583(9)$ & $-0.2120(5)$ & $2.2(2)$ \\
\hline $\mathrm{C}(3)$ & $0.6215(9)$ & $-0.0749(8)$ & $-0.2372(5)$ & $1.9(2)$ \\
\hline $\mathrm{C}(4)$ & $0.6919(9)$ & $-0.1061(8)$ & $-0.2917(5)$ & $1.9(2)$ \\
\hline $\mathrm{C}(5)$ & $0.689(1)$ & $-0.2106(9)$ & $-0.2996(5)$ & $2.5(2)$ \\
\hline$C(6)$ & $0.3913(9)$ & $-0.0567(8)$ & $-0.4110(5)$ & $1.9(3)$ \\
\hline$C(7)$ & $0.4941(9)$ & $-0.1250(8)$ & $-0.4313(5)$ & $2.3(3)$ \\
\hline $\mathrm{C}(8)$ & $0.5876(8)$ & $-0.0784(8)$ & $-0.4201(5)$ & $2.2(2)$ \\
\hline$C(9)$ & $0.5449(10)$ & $0.0155(8)$ & $-0.3900(5)$ & $1.9(3)$ \\
\hline$C(10)$ & $0.4242(9)$ & $0.0299(8)$ & $-0.3869(5)$ & $1.7(3)$ \\
\hline $\mathrm{C}(11)$ & $0.2754(10)$ & $-0.0688(9)$ & $-0.4277(6)$ & $2.2(3)$ \\
\hline$C(12)$ & $0.497(1)$ & $-0.2149(8)$ & $-0.4693(5)$ & $2.2(3)$ \\
\hline$C(13)$ & $0.7111(10)$ & $-0.1164(9)$ & $-0.4430(5)$ & $2.9(3)$ \\
\hline$C(14)$ & $0.614(1)$ & $0.0943(8)$ & $-0.3746(6)$ & $2.4(3)$ \\
\hline$C(15)$ & $0.3482(9)$ & $0.1235(8)$ & $-0.3662(5)$ & $2.4(3)$ \\
\hline$C(16)$ & $0.4776(10)$ & $-0.4347(8)$ & $-0.3608(5)$ & $2.1(3)$ \\
\hline$C(17)$ & $0.423(1)$ & $-0.5163(9)$ & $-0.3652(7)$ & $3.2(3)$ \\
\hline $\mathrm{C}(18)$ & $0.474(1)$ & $-0.602(1)$ & $-0.3944(8)$ & $4.8(5)$ \\
\hline$C(19)$ & $0.587(1)$ & $-0.6132(9)$ & $-0.4185(7)$ & $3.4(4)$ \\
\hline $\mathrm{C}(20)$ & $0.645(1)$ & $-0.5348(9)$ & $-0.4131(6)$ & $2.8(3)$ \\
\hline $\mathrm{C}(21)$ & $0.592(1)$ & $-0.4466(8)$ & $-0.3864(5)$ & $2.4(3)$ \\
\hline $\mathrm{C}(22)$ & $0.2211(10)$ & $-0.2937(8)$ & $-0.3373(5)$ & $2.4(3)$ \\
\hline $\mathrm{C}(23)$ & $0.127(1)$ & $-0.2607(9)$ & $-0.2940(5)$ & $2.5(3)$ \\
\hline $\mathrm{C}(24)$ & $0.016(1)$ & $-0.2437(9)$ & $-0.3120(7)$ & $2.9(3)$ \\
\hline$C(25)$ & $-0.003(1)$ & $-0.266(1)$ & $-0.3698(7)$ & $3.8(4)$ \\
\hline$C(26)$ & $0.085(1)$ & $-0.302(1)$ & $-0.4137(7)$ & $4.2(4)$ \\
\hline $\mathrm{C}(27)$ & $0.195(1)$ & $-0.317(1)$ & $-0.3957(5)$ & $2.6(3)$ \\
\hline $\mathrm{C}(28)$ & $0.3787(9)$ & $-0.3566(8)$ & $-0.2184(5)$ & $1.3(2)$ \\
\hline$C(29)$ & $0.3338(10)$ & $-0.2901(8)$ & $-0.1700(5)$ & $1.9(3)$ \\
\hline$C(30)$ & $0.324(1)$ & $-0.3253(10)$ & $-0.1071(5)$ & $2.7(3)$ \\
\hline $\mathrm{C}(31)$ & $0.363(1)$ & $-0.4256(10)$ & $-0.0948(5)$ & $2.9(3)$ \\
\hline$C(32)$ & $0.4068(10)$ & $-0.4908(9)$ & $-0.1414(5)$ & $2.4(3)$ \\
\hline $\mathrm{C}(33)$ & $0.4135(9)$ & $-0.4568(8)$ & $-0.2042(6)$ & $2.0(3)$ \\
\hline $\mathrm{C}(34)$ & $0.349(1)$ & $-0.0040(10)$ & $-0.1921(6)$ & $2.6(3)$ \\
\hline$C(35)$ & $0.2402(10)$ & $-0.0383(8)$ & $-0.2705(6)$ & $2.5(3)$ \\
\hline$C(36)$ & $-0.127(1)$ & $-0.2574(9)$ & $0.2484(7)$ & $2.9(3)$ \\
\hline $\mathrm{C}(37)$ & $-0.108(1)$ & $-0.339(1)$ & $0.2898(6)$ & $3.6(3)$ \\
\hline $\mathrm{C}(38)$ & $-0.140(1)$ & $-0.4226(10)$ & $0.2633(7)$ & $4.2(3)$ \\
\hline C(39) & $-0.180(1)$ & $-0.391(1)$ & $0.2045(7)$ & $3.4(4)$ \\
\hline $\mathrm{C}(40)$ & $-0.173(1)$ & $-0.2875(10)$ & $0.1943(7)$ & $3.2(4)$ \\
\hline $\mathrm{C}(41)$ & $0.1801(9)$ & $-0.4275(8)$ & $0.1030(5)$ & $1.6(2)$ \\
\hline $\mathrm{C}(42)$ & $0.1370(9)$ & $-0.5146(9)$ & $0.1238(5)$ & $2.1(3)$ \\
\hline $\mathrm{C}(43)$ & $0.018(1)$ & $-0.5011(9)$ & $0.1113(6)$ & $2.7(3)$ \\
\hline$C(44)$ & $-0.007(1)$ & $-0.4077(9)$ & $0.0830(6)$ & $2.6(3)$ \\
\hline$C(45)$ & $0.090(1)$ & $-0.3594(8)$ & $0.0797(5)$ & $2.0(3)$ \\
\hline$C(46)$ & $0.3015(9)$ & $-0.4105(8)$ & $0.0967(6)$ & $2.2(3)$ \\
\hline $\mathrm{C}(47)$ & $0.202(1)$ & $-0.6128(8)$ & $0.1447(6)$ & $2.9(3)$ \\
\hline
\end{tabular}




\begin{tabular}{|c|c|c|c|c|}
\hline$C(48)$ & $-0.052(1)$ & $-0.585(1)$ & $0.1188(8)$ & $4.2(4)$ \\
\hline C(49) & $-0.117(1)$ & $-0.372(1)$ & $0.0525(7)$ & $4.5(4)$ \\
\hline$C(50)$ & $0.105(1)$ & $-0.2624(9)$ & $0.0452(5)$ & $3.1(3)$ \\
\hline $\mathrm{C}(51)$ & $0.0573(9)$ & $-0.0556(8)$ & $0.1686(5)$ & $1.9(2)$ \\
\hline $\mathrm{C}(52)$ & $-0.0338(10)$ & $-0.0429(8)$ & $0.1305(5)$ & $2.6(3)$ \\
\hline $\mathrm{C}(53)$ & $-0.073(1)$ & $0.0507(9)$ & $0.1024(5)$ & $3.1(3)$ \\
\hline $\mathrm{C}(54)$ & $-0.022(1)$ & $0.1316(9)$ & $0.1133(6)$ & $2.9(3)$ \\
\hline$C(55)$ & $0.067(1)$ & $0.1217(9)$ & $0.1511(6)$ & $2.8(3)$ \\
\hline$C(56)$ & $0.1044(10)$ & $0.0284(9)$ & $0.1779(5)$ & $2.5(3)$ \\
\hline$C(57)$ & $0.3025(9)$ & $-0.1944(8)$ & $0.1909(5)$ & $2.4(3)$ \\
\hline $\mathrm{C}(58)$ & $0.3431(10)$ & $-0.1516(9)$ & $0.1370(6)$ & $1.9(3)$ \\
\hline C(59) & $0.4567(10)$ & $-0.1572(9)$ & $0.1179(6)$ & $2.5(3)$ \\
\hline$C(60)$ & $0.5366(10)$ & $-0.2072(9)$ & $0.1542(7)$ & $3.1(3)$ \\
\hline $\mathrm{C}(61)$ & $0.502(1)$ & $-0.2480(9)$ & $0.2105(6)$ & $2.3(3)$ \\
\hline$C(62)$ & $0.3852(9)$ & $-0.2418(8)$ & $0.2296(5)$ & $1.8(3)$ \\
\hline$C(63)$ & $0.0964(9)$ & $-0.1556(8)$ & $0.3098(5)$ & $1.8(2)$ \\
\hline$C(64)$ & $0.0494(9)$ & $-0.0597(9)$ & $0.3304(5)$ & $2.6(3)$ \\
\hline$C(65)$ & $0.0369(10)$ & $-0.0355(10)$ & $0.3936(6)$ & $2.9(3)$ \\
\hline$C(66)$ & $0.0726(10)$ & $-0.109(1)$ & $0.4364(5)$ & $3.0(3)$ \\
\hline$C(67)$ & $0.119(1)$ & $-0.2032(9)$ & $0.4177(5)$ & $2.8(3)$ \\
\hline $\mathrm{C}(68)$ & $0.129(1)$ & $-0.2285(9)$ & $0.3552(5)$ & $3.2(3)$ \\
\hline C(69) & $0.098(1)$ & $-0.503(1)$ & $0.3215(7)$ & $4.3(4)$ \\
\hline $\mathrm{C}(70)$ & $0.250(1)$ & $-0.4638(9)$ & $0.2521(6)$ & $3.0(3)$ \\
\hline $\mathrm{C}(71)$ & $0.588(1)$ & $-0.3460(10)$ & $-0.0074(6)$ & $3.4(3)$ \\
\hline $\mathrm{C}(72)$ & $0.582(1)$ & $-0.2419(10)$ & $-0.0375(6)$ & $3.1(3)$ \\
\hline $\mathrm{C}(73)$ & $0.661(1)$ & $-0.095(1)$ & $-0.0360(6)$ & $3.6(3)$ \\
\hline $\mathrm{C}(74)$ & $0.736(1)$ & $-0.044(1)$ & $0.0008(7)$ & $4.8(3)$ \\
\hline$C(75)$ & $0.115(2)$ & $0.395(2)$ & $-0.477(1)$ & $10.2(7)$ \\
\hline$C(76)$ & $0.202(4)$ & $0.397(3)$ & $-0.463(2)$ & $6.8(8)$ \\
\hline$C(100)$ & 0.6382 & -0.1583 & -0.2581 & 0.2000 \\
\hline $\mathrm{C}(101)$ & 0.4882 & -0.0425 & -0.4078 & 0.2000 \\
\hline$C(102)$ & -0.1455 & -0.3395 & 0.2415 & 0.2000 \\
\hline$C(103)$ & 0.0822 & -0.4421 & 0.1005 & 0.2000 \\
\hline $\mathrm{H}(1)$ & 0.5930 & -0.3040 & -0.2426 & 2.8428 \\
\hline $\mathrm{H}(2)$ & 0.5293 & -0.1611 & -0.1749 & 2.5158 \\
\hline $\mathrm{H}(3)$ & 0.6029 & -0.0094 & -0.2194 & 2.4582 \\
\hline $\mathrm{H}(4)$ & 0.7356 & -0.0659 & -0.3180 & 1.7165 \\
\hline $\mathrm{H}(5)$ & 0.7271 & -0.2523 & -0.3333 & 2.2039 \\
\hline $\mathrm{H}(6)$ & 0.2223 & -0.0559 & -0.3915 & 2.6069 \\
\hline $\mathrm{H}(7)$ & 0.2528 & -0.0181 & -0.4583 & 2.6069 \\
\hline $\mathrm{H}(8)$ & 0.2779 & -0.1317 & -0.4424 & 2.6069 \\
\hline $\mathrm{H}(9)$ & 0.4229 & -0.2295 & -0.4690 & 2.5820 \\
\hline $\mathrm{H}(10)$ & 0.5279 & -0.2045 & -0.5106 & 2.5820 \\
\hline $\mathrm{H}(11)$ & 0.5449 & -0.2710 & -0.4516 & 2.5820 \\
\hline $\mathrm{H}(12)$ & 0.7326 & -0.1829 & -0.4268 & 3.4980 \\
\hline $\mathrm{H}(13)$ & 0.7195 & -0.1185 & -0.4868 & 3.4980 \\
\hline
\end{tabular}




\begin{tabular}{|c|c|c|c|c|}
\hline $\mathrm{H}(14)$ & 0.7592 & -0.0744 & -0.4290 & 3.4980 \\
\hline $\mathrm{H}(15)$ & 0.6926 & 0.0672 & -0.3793 & 2.8901 \\
\hline $\mathrm{H}(16)$ & 0.5983 & 0.1498 & -0.4014 & 2.8901 \\
\hline $\mathrm{H}(17)$ & 0.5926 & 0.1141 & -0.3326 & 2.8901 \\
\hline $\mathrm{H}(18)$ & 0.3645 & 0.1389 & -0.3257 & 2.9346 \\
\hline $\mathrm{H}(19)$ & 0.3685 & 0.1772 & -0.3940 & 2.9346 \\
\hline $\mathrm{H}(20)$ & 0.2726 & 0.1189 & -0.3676 & 2.9346 \\
\hline $\mathrm{H}(21)$ & -0.1159 & -0.1938 & 0.2565 & 3.5369 \\
\hline $\mathrm{H}(22)$ & -0.0756 & -0.3376 & 0.3295 & 4.3774 \\
\hline $\mathrm{H}(23)$ & -0.1360 & -0.4867 & 0.2824 & 5.0360 \\
\hline $\mathrm{H}(24)$ & -0.2092 & -0.4307 & 0.1798 & 4.0758 \\
\hline $\mathrm{H}(25)$ & -0.1904 & -0.2481 & 0.1600 & 3.8910 \\
\hline $\mathrm{H}(26)$ & 0.3477 & -0.4646 & 0.1150 & 2.6372 \\
\hline $\mathrm{H}(27)$ & 0.3274 & -0.4052 & 0.0536 & 2.6372 \\
\hline $\mathrm{H}(28)$ & 0.3064 & -0.3496 & 0.1163 & 2.6372 \\
\hline $\mathrm{H}(29)$ & 0.1693 & -0.6302 & 0.1840 & 3.5188 \\
\hline $\mathrm{H}(30)$ & 0.1988 & -0.6638 & 0.1157 & 3.5188 \\
\hline $\mathrm{H}(31)$ & 0.2797 & -0.6063 & 0.1471 & 3.5188 \\
\hline $\mathrm{H}(32)$ & -0.1254 & -0.5620 & 0.1396 & 5.0047 \\
\hline $\mathrm{H}(33)$ & -0.0602 & -0.6080 & 0.0790 & 5.0047 \\
\hline $\mathrm{H}(34)$ & -0.0142 & -0.6388 & 0.1425 & 5.0047 \\
\hline $\mathrm{H}(35)$ & -0.1501 & -0.3069 & 0.0669 & 5.3531 \\
\hline $\mathrm{H}(36)$ & -0.0984 & -0.3684 & 0.0087 & 5.3531 \\
\hline $\mathrm{H}(37)$ & -0.1692 & -0.4169 & 0.0615 & 5.3531 \\
\hline $\mathrm{H}(38)$ & 0.1330 & -0.2193 & 0.0706 & 3.6998 \\
\hline H(39) & 0.1524 & -0.2767 & 0.0085 & 3.6998 \\
\hline $\mathrm{H}(40)$ & 0.0303 & -0.2286 & 0.0337 & 3.6998 \\
\hline $\mathrm{H}(41)$ & 0.3480 & -0.5099 & -0.3482 & 3.8177 \\
\hline $\mathrm{H}(42)$ & 0.4353 & -0.6568 & -0.3961 & 5.7457 \\
\hline $\mathrm{H}(43)$ & 0.6223 & -0.6724 & -0.4400 & 4.0561 \\
\hline $\mathrm{H}(44)$ & 0.7235 & -0.5404 & -0.4282 & 3.3751 \\
\hline $\mathrm{H}(45)$ & 0.6327 & -0.3922 & -0.3847 & 2.8954 \\
\hline $\mathrm{H}(46)$ & 0.1363 & -0.2522 & -0.2520 & 2.9473 \\
\hline $\mathrm{H}(47)$ & -0.0436 & -0.2179 & -0.2831 & 3.4393 \\
\hline $\mathrm{H}(48)$ & -0.0786 & -0.2575 & -0.3819 & 4.5545 \\
\hline H(49) & 0.0725 & -0.3148 & -0.4559 & 4.9892 \\
\hline $\mathrm{H}(50)$ & 0.2571 & -0.3471 & -0.4237 & 3.1163 \\
\hline $\mathrm{H}(51)$ & 0.3087 & -0.2211 & -0.1785 & 2.2680 \\
\hline $\mathrm{H}(52)$ & 0.2900 & -0.2824 & -0.0750 & 3.1888 \\
\hline $\mathrm{H}(53)$ & 0.3591 & -0.4499 & -0.0528 & 3.4512 \\
\hline $\mathrm{H}(54)$ & 0.4318 & -0.5599 & -0.1338 & 2.8901 \\
\hline $\mathrm{H}(55)$ & 0.4449 & -0.5019 & -0.2356 & 2.3723 \\
\hline $\mathrm{H}(56)$ & 0.4225 & -0.0076 & -0.1793 & 3.1748 \\
\hline $\mathrm{H}(57)$ & 0.3126 & 0.0639 & -0.1940 & 3.1748 \\
\hline $\mathrm{H}(58)$ & 0.3045 & -0.0406 & -0.1641 & 3.1748 \\
\hline $\mathrm{H}(59)$ & 0.2065 & 0.0300 & -0.2706 & 3.0458 \\
\hline
\end{tabular}




$\begin{array}{lrrrl}\mathrm{H}(60) & 0.2422 & -0.0667 & -0.3093 & 3.0458 \\ \mathrm{H}(61) & 0.1978 & -0.0738 & -0.2399 & 3.0458 \\ \mathrm{H}(62) & -0.0690 & -0.0984 & 0.1255 & 3.0784 \\ \mathrm{H}(63) & -0.1311 & 0.0555 & 0.0750 & 3.7121 \\ \mathrm{H}(64) & -0.0499 & 0.1935 & 0.0949 & 3.5188 \\ \mathrm{H}(65) & 0.1031 & 0.1780 & 0.1579 & 3.4086 \\ \mathrm{H}(66) & 0.1606 & 0.0247 & 0.2046 & 2.9777 \\ \mathrm{H}(67) & 0.2880 & -0.1169 & 0.1109 & 2.3339 \\ \mathrm{H}(68) & 0.4800 & -0.1280 & 0.0786 & 3.0238 \\ \mathrm{H}(69) & 0.6165 & -0.2113 & 0.1414 & 3.6893 \\ \mathrm{H}(70) & 0.5578 & -0.2811 & 0.2375 & 2.7490 \\ \mathrm{H}(71) & 0.3616 & -0.2697 & 0.2688 & 2.1259 \\ \mathrm{H}(72) & 0.0274 & -0.0113 & 0.2987 & 3.0970 \\ \mathrm{H}(73) & 0.0034 & 0.0313 & 0.4066 & 3.5237 \\ \mathrm{H}(74) & 0.0670 & -0.0936 & 0.4794 & 3.6563 \\ \mathrm{H}(75) & 0.1424 & -0.2537 & 0.4474 & 3.3414 \\ \mathrm{H}(76) & 0.1607 & -0.2959 & 0.3445 & 3.8749 \\ \mathrm{H}(77) & 0.0146 & -0.4987 & 0.3272 & 5.2156 \\ \mathrm{H}(78) & 0.1287 & -0.5726 & 0.3183 & 5.2156 \\ \mathrm{H}(79) & 0.1228 & -0.4729 & 0.3530 & 5.2156 \\ \mathrm{H}(80) & 0.2831 & -0.5330 & 0.2521 & 3.6158 \\ \mathrm{H}(81) & 0.2725 & -0.4325 & 0.2151 & 3.6158 \\ \mathrm{H}(82) & 0.2765 & -0.4328 & 0.2864 & 3.6158 \\ \mathrm{H}(83) & 0.5701 & -0.3412 & 0.0370 & 4.4737 \\ \mathrm{H}(84) & 0.5409 & -0.3859 & -0.0234 & 4.4737 \\ \mathrm{H}(85) & 0.6667 & -0.3816 & -0.0133 & 4.4737 \\ \mathrm{H}(86) & 0.5038 & -0.2067 & -0.0307 & 3.7644 \\ \mathrm{H}(87) & 0.5998 & -0.2473 & -0.0813 & 3.7644 \\ \mathrm{H}(88) & 0.6912 & -0.0965 & -0.0792 & 4.6575 \\ \mathrm{H}(89) & 0.5853 & -0.0536 & -0.0351 & 4.6575 \\ \mathrm{H}(90) & 0.8096 & -0.0832 & 0.0002 & 4.9778 \\ \mathrm{H}(91) & 0.7421 & 0.0220 & -0.0135 & 4.9778 \\ \mathrm{H}(92) & 0.7036 & -0.0394 & 0.0439 & 4.9778 \\ & & & & \end{array}$

$B_{\text {eq }}=8 / 3 \pi^{2}\left(U_{11}\left(a a^{*}\right)^{2}+U_{22}\left(b b^{*}\right)^{2}+U_{33}\left(c c^{*}\right)^{2}+2 U_{12}\left(a a^{*} b b^{*}\right) \cos \gamma+2 U_{13}\left(a a^{*} c c^{*}\right) \cos \beta+\right.$ $\left.2 \mathrm{U}_{23}\left(\mathrm{bb}^{*} \mathrm{cc} *\right) \cos \alpha\right)$

Table 19. Anisotropic Displacement Parameters for $\mathrm{CpCp} * \mathrm{Hf}\left(\mathrm{SnPh}_{3}\right) \mathrm{NMe}_{2} \bullet 0.75\left(\mathrm{C}_{4} \mathrm{H}_{10} \mathrm{O}\right)(\mathbf{8})$

$\begin{array}{llllllc}\text { atom } & \mathrm{U}_{11} & \mathrm{U}_{22} & \mathrm{U}_{33} & \mathrm{U}_{12} & \mathrm{U}_{13} & \mathrm{U}_{23} \\ \mathrm{Hf}(1) & 0.0143(3) & 0.0159(3) & 0.0196(3) & -0.0017(2) & -0.0040(2) & 0.0014(2) \\ \mathrm{Hf}(2) & 0.0157(3) & 0.0169(3) & 0.0318(3) & -0.0017(2) & -0.0014(2) & -0.0001(2) \\ \mathrm{Sn}(1) & 0.0210(4) & 0.0178(4) & 0.0212(4) & -0.0041(3) & -0.0015(3) & 0.0010(3) \\ \mathrm{Sn}(2) & 0.0185(4) & 0.0164(4) & 0.0216(4) & -0.0023(3) & -0.0029(3) & 0.0008(3) \\ \mathrm{N}(1) & 0.026(5) & 0.017(5) & 0.031(6) & -0.005(4) & -0.004(4) & -0.001(4) \\ \mathrm{N}(2) & 0.021(6) & 0.021(5) & 0.053(7) & -0.002(4) & -0.001(5) & -0.005(5)\end{array}$




\begin{tabular}{|c|c|c|c|c|c|c|}
\hline$C(6)$ & $0.024(7)$ & $0.029(7)$ & $0.026(6)$ & $-0.003(5)$ & $-0.017(5)$ & $0.003(5)$ \\
\hline$C(7)$ & $0.027(7)$ & $0.023(6)$ & $0.026(7)$ & $-0.003(5)$ & $-0.015(5)$ & $0.009(5)$ \\
\hline $\mathrm{C}(8)$ & $0.008(5)$ & $0.019(6)$ & $0.030(6)$ & $0.002(4)$ & $-0.003(5)$ & $0.000(5)$ \\
\hline$C(9)$ & $0.031(7)$ & $0.022(6)$ & $0.019(6)$ & $-0.007(5)$ & $-0.007(5)$ & $0.007(5)$ \\
\hline$C(10)$ & $0.026(7)$ & $0.015(6)$ & $0.031(7)$ & $-0.007(5)$ & $-0.003(5)$ & $0.016(5)$ \\
\hline $\mathrm{C}(11)$ & $0.024(7)$ & $0.036(7)$ & $0.041(8)$ & $-0.008(6)$ & $-0.014(6)$ & $0.005(6)$ \\
\hline$C(12)$ & $0.045(8)$ & $0.030(7)$ & $0.013(6)$ & $-0.009(6)$ & $-0.004(5)$ & $0.003(5)$ \\
\hline$C(13)$ & $0.026(7)$ & $0.043(8)$ & $0.032(7)$ & $-0.005(6)$ & $0.005(5)$ & $0.005(6)$ \\
\hline$C(14)$ & $0.037(8)$ & $0.012(6)$ & $0.046(8)$ & $-0.009(5)$ & $-0.017(6)$ & $0.003(5)$ \\
\hline$C(15)$ & $0.026(7)$ & $0.021(6)$ & $0.036(7)$ & $0.000(5)$ & $-0.006(5)$ & $0.009(5)$ \\
\hline$C(16)$ & $0.034(7)$ & $0.012(6)$ & $0.036(7)$ & $-0.003(5)$ & $-0.008(6)$ & $-0.007(5)$ \\
\hline$C(17)$ & $0.036(8)$ & $0.030(8)$ & $0.061(10)$ & $-0.002(6)$ & $0.005(7)$ & $0.001(7)$ \\
\hline$C(18)$ & $0.06(1)$ & $0.05(1)$ & $0.09(1)$ & $-0.028(9)$ & $0.010(9)$ & $-0.031(9)$ \\
\hline$C(19)$ & $0.049(9)$ & $0.028(8)$ & $0.060(10)$ & $-0.009(7)$ & $0.016(7)$ & $-0.003(7)$ \\
\hline$C(20)$ & $0.028(7)$ & $0.038(8)$ & $0.051(9)$ & $-0.003(6)$ & $0.020(6)$ & $-0.012(7)$ \\
\hline $\mathrm{C}(21)$ & $0.048(8)$ & $0.011(6)$ & $0.028(7)$ & $-0.010(5)$ & $-0.011(6)$ & $-0.005(5)$ \\
\hline $\mathrm{C}(22)$ & $0.034(7)$ & $0.013(6)$ & $0.026(7)$ & $-0.002(5)$ & $0.002(5)$ & $0.009(5)$ \\
\hline $\mathrm{C}(23)$ & $0.030(7)$ & $0.029(7)$ & $0.037(7)$ & $-0.009(6)$ & $0.001(6)$ & $0.000(6)$ \\
\hline$C(24)$ & $0.018(7)$ & $0.032(8)$ & $0.06(1)$ & $-0.002(6)$ & $-0.001(6)$ & $-0.006(7)$ \\
\hline$C(25)$ & $0.025(8)$ & $0.058(10)$ & $0.06(1)$ & $-0.009(7)$ & $-0.013(7)$ & $0.016(8)$ \\
\hline$C(26)$ & $0.039(9)$ & $0.10(1)$ & $0.052(10)$ & $-0.036(9)$ & $-0.018(7)$ & $0.020(9)$ \\
\hline$C(27)$ & $0.028(7)$ & $0.060(9)$ & $0.028(7)$ & $-0.020(6)$ & $0.007(5)$ & $0.003(6)$ \\
\hline $\mathrm{C}(28)$ & $0.023(6)$ & $0.022(6)$ & $0.016(6)$ & $-0.015(5)$ & $-0.005(5)$ & $0.009(5)$ \\
\hline $\mathrm{C}(29)$ & $0.031(7)$ & $0.025(7)$ & $0.033(7)$ & $-0.010(5)$ & $-0.012(5)$ & $0.009(5)$ \\
\hline$C(30)$ & $0.033(8)$ & $0.051(9)$ & $0.023(7)$ & $-0.009(6)$ & $0.000(5)$ & $-0.005(6)$ \\
\hline $\mathrm{C}(31)$ & $0.035(8)$ & $0.048(9)$ & $0.025(7)$ & $-0.025(6)$ & $-0.011(6)$ & $0.011(6)$ \\
\hline$C(32)$ & $0.030(7)$ & $0.030(7)$ & $0.035(7)$ & $-0.012(6)$ & $-0.013(6)$ & $0.011(6)$ \\
\hline $\mathrm{C}(33)$ & $0.021(7)$ & $0.022(7)$ & $0.043(8)$ & $-0.006(5)$ & $0.001(5)$ & $0.005(6)$ \\
\hline$C(34)$ & $0.025(7)$ & $0.046(8)$ & $0.046(8)$ & $-0.019(6)$ & $0.000(6)$ & $-0.008(7)$ \\
\hline$C(35)$ & $0.026(7)$ & $0.022(7)$ & $0.044(8)$ & $0.006(5)$ & $0.008(6)$ & $0.002(6)$ \\
\hline$C(36)$ & $0.022(7)$ & $0.020(7)$ & $0.066(10)$ & $0.008(5)$ & $0.014(6)$ & $-0.006(7)$ \\
\hline$C(37)$ & $0.027(7)$ & $0.050(9)$ & $0.042(8)$ & $0.000(6)$ & $0.014(6)$ & $0.002(7)$ \\
\hline$C(38)$ & $0.024(7)$ & $0.038(8)$ & $0.07(1)$ & $-0.010(6)$ & $0.006(7)$ & $0.013(7)$ \\
\hline$C(39)$ & $0.017(7)$ & $0.053(9)$ & $0.07(1)$ & $-0.015(6)$ & $0.005(7)$ & $-0.011(8)$ \\
\hline$C(40)$ & $0.029(8)$ & $0.033(8)$ & $0.08(1)$ & $-0.010(6)$ & $-0.003(7)$ & $0.008(8)$ \\
\hline $\mathrm{C}(41)$ & $0.022(6)$ & $0.022(6)$ & $0.020(6)$ & $0.000(5)$ & $-0.003(5)$ & $-0.006(5)$ \\
\hline$C(42)$ & $0.017(6)$ & $0.033(7)$ & $0.038(7)$ & $-0.002(5)$ & $-0.001(5)$ & $-0.009(6)$ \\
\hline$C(43)$ & $0.026(7)$ & $0.026(7)$ & $0.052(8)$ & $-0.005(5)$ & $-0.010(6)$ & $-0.004(6)$ \\
\hline$C(44)$ & $0.036(8)$ & $0.032(8)$ & $0.046(8)$ & $-0.010(6)$ & $-0.021(6)$ & $-0.009(6)$ \\
\hline$C(45)$ & $0.039(7)$ & $0.020(6)$ & $0.020(6)$ & $-0.005(5)$ & $-0.002(5)$ & $-0.005(5)$ \\
\hline$C(46)$ & $0.015(6)$ & $0.028(7)$ & $0.046(8)$ & $0.002(5)$ & $-0.002(5)$ & $-0.006(6)$ \\
\hline $\mathrm{C}(47)$ & $0.042(8)$ & $0.019(6)$ & $0.041(8)$ & $0.004(6)$ & $-0.009(6)$ & $-0.010(6)$ \\
\hline$C(48)$ & $0.027(8)$ & $0.046(9)$ & $0.11(1)$ & $-0.011(7)$ & $-0.014(8)$ & $-0.015(9)$ \\
\hline$C(49)$ & $0.040(9)$ & $0.07(1)$ & $0.08(1)$ & $0.001(8)$ & $-0.037(8)$ & $-0.005(9)$ \\
\hline$C(50)$ & $0.045(8)$ & $0.038(8)$ & $0.022(7)$ & $-0.003(6)$ & $0.002(6)$ & $0.002(6)$ \\
\hline$C(51)$ & $0.020(6)$ & $0.023(6)$ & $0.026(6)$ & $-0.006(5)$ & $0.001(5)$ & $-0.002(5)$ \\
\hline
\end{tabular}




$\begin{array}{lllllrr}\mathrm{C}(52) & 0.028(7) & 0.026(7) & 0.028(7) & -0.008(5) & -0.006(5) & 0.006(5) \\ \mathrm{C}(53) & 0.038(8) & 0.032(7) & 0.030(7) & 0.000(6) & -0.008(6) & 0.000(6) \\ \mathrm{C}(54) & 0.049(9) & 0.016(6) & 0.042(8) & 0.004(6) & 0.002(6) & 0.009(6) \\ \mathrm{C}(55) & 0.039(8) & 0.029(7) & 0.041(8) & -0.019(6) & -0.004(6) & -0.003(6) \\ \mathrm{C}(56) & 0.030(7) & 0.026(7) & 0.037(7) & -0.004(5) & -0.012(6) & -0.001(6) \\ \mathrm{C}(57) & 0.022(6) & 0.027(7) & 0.026(7) & -0.002(5) & -0.013(5) & -0.010(5) \\ \mathrm{C}(58) & 0.027(7) & 0.026(7) & 0.036(7) & 0.000(5) & -0.007(6) & 0.004(6) \\ \mathrm{C}(59) & 0.012(6) & 0.037(8) & 0.056(9) & -0.002(5) & 0.006(6) & -0.004(6) \\ \mathrm{C}(60) & 0.015(7) & 0.036(8) & 0.07(1) & -0.005(6) & 0.003(6) & -0.005(7) \\ \mathrm{C}(61) & 0.021(7) & 0.030(7) & 0.056(9) & 0.003(6) & -0.020(6) & -0.013(6) \\ \mathrm{C}(62) & 0.020(7) & 0.030(7) & 0.038(7) & -0.006(5) & -0.016(5) & -0.003(6) \\ \mathrm{C}(63) & 0.021(6) & 0.020(6) & 0.024(6) & -0.004(5) & -0.002(5) & -0.004(5) \\ \mathrm{C}(64) & 0.017(6) & 0.035(7) & 0.025(7) & -0.007(5) & -0.004(5) & -0.002(5) \\ \mathrm{C}(65) & 0.019(7) & 0.044(8) & 0.048(8) & -0.010(6) & -0.003(6) & -0.017(7) \\ \mathrm{C}(66) & 0.026(7) & 0.063(10) & 0.019(7) & -0.006(7) & 0.006(5) & -0.014(6) \\ \mathrm{C}(67) & 0.048(9) & 0.037(8) & 0.019(7) & -0.001(6) & 0.008(6) & -0.001(6) \\ \mathrm{C}(68) & 0.052(9) & 0.029(7) & 0.028(7) & -0.018(6) & 0.006(6) & 0.000(6) \\ \mathrm{C}(69) & 0.06(1) & 0.047(9) & 0.056(10) & -0.015(8) & -0.011(8) & 0.032(8) \\ \mathrm{C}(70) & 0.036(8) & 0.030(7) & 0.054(9) & 0.018(6) & -0.017(7) & -0.001(6)\end{array}$

The general temperature factor expression:

$\exp \left(-2 \pi^{2}\left(a^{*}{ }^{2} U_{11} h^{2}+b^{*}{ }^{2} U_{22} k^{2}+c^{*} U_{33} l^{2}+2 a * b * U_{12} h k+2 a * c * U_{13} h l+2 b * c * U_{23} k l\right)\right)$

Table 20. Bond Lengths $(\AA)$ for $\mathrm{CpCp} * \mathrm{Hf}\left(\mathrm{SnPh}_{3}\right) \mathrm{NMe}_{2} \bullet 0.75\left(\mathrm{C}_{4} \mathrm{H}_{10} \mathrm{O}\right)(\mathbf{8})$

$\begin{array}{llllll}\text { atom } & \text { atom } & \text { distance } & \text { atom } & \text { atom } & \text { distance } \\ \mathrm{Hf}(1) & \mathrm{Sn}(1) & 2.9694(8) & \mathrm{Hf}(1) & \mathrm{N}(1) & 2.065(9) \\ \mathrm{Hf}(1) & \mathrm{C}(1) & 2.54(1) & \mathrm{Hf}(1) & \mathrm{C}(2) & 2.51(1) \\ \mathrm{Hf}(1) & \mathrm{C}(3) & 2.48(1) & \mathrm{Hf}(1) & \mathrm{C}(4) & 2.49(1) \\ \mathrm{Hf}(1) & \mathrm{C}(5) & 2.55(1) & \mathrm{Hf}(1) & \mathrm{C}(6) & 2.56(1) \\ \mathrm{Hf}(1) & \mathrm{C}(7) & 2.57(1) & \mathrm{Hf}(1) & \mathrm{C}(8) & 2.54(1) \\ \mathrm{Hf}(1) & \mathrm{C}(9) & 2.47(1) & \mathrm{Hf}(1) & \mathrm{C}(10) & 2.532(10) \\ \mathrm{Hf}(1) & \mathrm{C}(100) & 2.2098(4) & \mathrm{Hf}(1) & \mathrm{C}(101) & 2.2257(4) \\ \mathrm{Hf}(2) & \mathrm{Sn}(2) & 2.9658(8) & \mathrm{Hf}(2) & \mathrm{N}(2) & 2.068(10) \\ \mathrm{Hf}(2) & \mathrm{C}(36) & 2.51(1) & \mathrm{Hf}(2) & \mathrm{C}(37) & 2.51(1) \\ \mathrm{Hf}(2) & \mathrm{C}(38) & 2.50(1) & \mathrm{Hf}(2) & \mathrm{C}(39) & 2.49(1) \\ \mathrm{Hf}(2) & \mathrm{C}(40) & 2.52(1) & \mathrm{Hf}(2) & \mathrm{C}(41) & 2.59(1) \\ \mathrm{Hf}(2) & \mathrm{C}(42) & 2.50(1) & \mathrm{Hf}(2) & \mathrm{C}(43) & 2.47(1) \\ \mathrm{Hf}(2) & \mathrm{C}(44) & 2.55(1) & \mathrm{Hf}(2) & \mathrm{C}(45) & 2.57(1) \\ \mathrm{Hf}(2) & \mathrm{C}(102) & 2.2047(5) & \mathrm{Hf}(2) & \mathrm{C}(103) & 2.2214(5) \\ \mathrm{Sn}(1) & \mathrm{C}(16) & 2.18(1) & \mathrm{Sn}(1) & \mathrm{C}(22) & 2.17(1) \\ \mathrm{Sn}(1) & \mathrm{C}(28) & 2.172(10) & \mathrm{Sn}(2) & \mathrm{C}(51) & 2.16(1) \\ \mathrm{Sn}(2) & \mathrm{C}(57) & 2.18(1) & \mathrm{Sn}(2) & \mathrm{C}(63) & 2.19(1) \\ \mathrm{O}(1) & \mathrm{C}(72) & 1.40(1) & \mathrm{O}(1) & \mathrm{C}(73) & 1.42(1) \\ \mathrm{O}(2) & \mathrm{O}(2) & 1.22(3) & \mathrm{O}(2) & \mathrm{C}(75) & 1.50(3) \\ \mathrm{N}(1) & \mathrm{C}(34) & 1.44(1) & \mathrm{N}(1) & \mathrm{C}(35) & 1.48(1) \\ & & \mathrm{S} 26 & & \end{array}$




\begin{tabular}{|c|c|c|c|c|c|}
\hline $\mathrm{N}(2)$ & $\mathrm{C}(69)$ & $1.47(2)$ & $\mathrm{N}(2)$ & $\mathrm{C}(70)$ & $1.46(2)$ \\
\hline$C(1)$ & $\mathrm{C}(2)$ & $1.40(2)$ & $\mathrm{C}(1)$ & $\mathrm{C}(5)$ & $1.40(2)$ \\
\hline $\mathrm{C}(1)$ & $\mathrm{C}(100)$ & $1.19(1)$ & $\mathrm{C}(2)$ & $\mathrm{C}(3)$ & $1.42(2)$ \\
\hline$C(2)$ & $\mathrm{C}(100)$ & $1.20(1)$ & $\mathrm{C}(3)$ & $\mathrm{C}(4)$ & $1.41(1)$ \\
\hline $\mathrm{C}(3)$ & $\mathrm{C}(100)$ & $1.21(1)$ & $C(4)$ & $C(5)$ & $1.44(2)$ \\
\hline $\mathrm{C}(4)$ & $\mathrm{C}(100)$ & $1.21(1)$ & $C(5)$ & $\mathrm{C}(100)$ & $1.21(1)$ \\
\hline$C(6)$ & $\mathrm{C}(7)$ & $1.45(2)$ & $\mathrm{C}(6)$ & $\mathrm{C}(10)$ & $1.42(2)$ \\
\hline$C(6)$ & $\mathrm{C}(11)$ & $1.50(1)$ & $\mathrm{C}(6)$ & $\mathrm{C}(101)$ & $1.21(1)$ \\
\hline$C(7)$ & $\mathrm{C}(8)$ & $1.41(1)$ & $C(7)$ & $\mathrm{C}(12)$ & $1.48(2)$ \\
\hline$C(7)$ & $\mathrm{C}(101)$ & $1.23(1)$ & $\mathrm{C}(8)$ & $\mathrm{C}(9)$ & $1.44(1)$ \\
\hline $\mathrm{C}(8)$ & $\mathrm{C}(13)$ & $1.52(1)$ & $\mathrm{C}(8)$ & $\mathrm{C}(101)$ & $1.214(10)$ \\
\hline $\mathrm{C}(9)$ & $C(10)$ & $1.42(1)$ & $\mathrm{C}(9)$ & $C(14)$ & $1.51(1)$ \\
\hline $\mathrm{C}(9)$ & $\mathrm{C}(101)$ & $1.21(1)$ & $\mathrm{C}(10)$ & $\mathrm{C}(15)$ & $1.48(2)$ \\
\hline$C(10)$ & $\mathrm{C}(101)$ & $1.21(1)$ & $C(16)$ & $\mathrm{C}(17)$ & $1.38(2)$ \\
\hline$C(16)$ & $\mathrm{C}(21)$ & $1.41(2)$ & $\mathrm{C}(17)$ & $\mathrm{C}(18)$ & $1.36(2)$ \\
\hline$C(18)$ & $\mathrm{C}(19)$ & $1.38(2)$ & $\mathrm{C}(19)$ & $\mathrm{C}(20)$ & $1.37(2)$ \\
\hline$C(20)$ & $\mathrm{C}(21)$ & $1.37(2)$ & $\mathrm{C}(22)$ & $\mathrm{C}(23)$ & $1.42(2)$ \\
\hline$C(22)$ & $\mathrm{C}(27)$ & $1.39(2)$ & $\mathrm{C}(23)$ & $\mathrm{C}(24)$ & $1.40(2)$ \\
\hline$C(24)$ & $\mathrm{C}(25)$ & $1.35(2)$ & $\mathrm{C}(25)$ & $\mathrm{C}(26)$ & $1.39(2)$ \\
\hline$C(26)$ & $\mathrm{C}(27)$ & $1.39(2)$ & $\mathrm{C}(28)$ & $\mathrm{C}(29)$ & $1.41(2)$ \\
\hline $\mathrm{C}(28)$ & $\mathrm{C}(33)$ & $1.39(1)$ & $\mathrm{C}(29)$ & $\mathrm{C}(30)$ & $1.43(2)$ \\
\hline$C(30)$ & $\mathrm{C}(31)$ & $1.39(2)$ & $\mathrm{C}(31)$ & $C(32)$ & $1.37(2)$ \\
\hline$C(32)$ & $C(33)$ & $1.42(2)$ & $C(36)$ & $C(37)$ & $1.40(2)$ \\
\hline$C(36)$ & $\mathrm{C}(40)$ & $1.44(2)$ & $C(36)$ & $\mathrm{C}(102)$ & $1.18(1)$ \\
\hline $\mathrm{C}(37)$ & $\mathrm{C}(38)$ & $1.41(2)$ & $\mathrm{C}(37)$ & $C(102)$ & $1.18(1)$ \\
\hline $\mathrm{C}(38)$ & $\mathrm{C}(39)$ & $1.43(2)$ & $\mathrm{C}(38)$ & $\mathrm{C}(102)$ & $1.20(1)$ \\
\hline$C(39)$ & $\mathrm{C}(40)$ & $1.43(2)$ & $\mathrm{C}(39)$ & $\mathrm{C}(102)$ & $1.22(1)$ \\
\hline$C(40)$ & $\mathrm{C}(102)$ & $1.26(1)$ & $\mathrm{C}(41)$ & $\mathrm{C}(42)$ & $1.41(2)$ \\
\hline$C(41)$ & $\mathrm{C}(45)$ & $1.42(1)$ & $\mathrm{C}(41)$ & $\mathrm{C}(46)$ & $1.50(1)$ \\
\hline $\mathrm{C}(41)$ & $\mathrm{C}(103)$ & $1.23(1)$ & $\mathrm{C}(42)$ & $\mathrm{C}(43)$ & $1.46(2)$ \\
\hline $\mathrm{C}(42)$ & $\mathrm{C}(47)$ & $1.51(2)$ & $\mathrm{C}(42)$ & $\mathrm{C}(103)$ & $1.21(1)$ \\
\hline$C(43)$ & $\mathrm{C}(44)$ & $1.39(2)$ & $\mathrm{C}(43)$ & $\mathrm{C}(48)$ & $1.51(2)$ \\
\hline$C(43)$ & $\mathrm{C}(103)$ & $1.20(1)$ & $\mathrm{C}(44)$ & $C(45)$ & $1.42(2)$ \\
\hline$C(44)$ & $\mathrm{C}(49)$ & $1.53(2)$ & $\mathrm{C}(44)$ & $\mathrm{C}(103)$ & $1.19(1)$ \\
\hline$C(45)$ & $\mathrm{C}(50)$ & $1.52(2)$ & $C(45)$ & $\mathrm{C}(103)$ & $1.21(1)$ \\
\hline $\mathrm{C}(51)$ & $C(52)$ & $1.41(1)$ & $\mathrm{C}(51)$ & $C(56)$ & $1.38(1)$ \\
\hline$C(52)$ & $\mathrm{C}(53)$ & $1.41(2)$ & $\mathrm{C}(53)$ & $\mathrm{C}(54)$ & $1.37(2)$ \\
\hline$C(54)$ & $\mathrm{C}(55)$ & $1.39(2)$ & $\mathrm{C}(55)$ & $C(56)$ & $1.40(2)$ \\
\hline$C(57)$ & $\mathrm{C}(58)$ & $1.37(2)$ & $\mathrm{C}(57)$ & $\mathrm{C}(62)$ & $1.42(1)$ \\
\hline$C(58)$ & $\mathrm{C}(59)$ & $1.37(2)$ & $\mathrm{C}(59)$ & $\mathrm{C}(60)$ & $1.37(2)$ \\
\hline$C(60)$ & $\mathrm{C}(61)$ & $1.38(2)$ & $\mathrm{C}(61)$ & $\mathrm{C}(62)$ & $1.41(2)$ \\
\hline$C(63)$ & $\mathrm{C}(64)$ & $1.39(1)$ & $\mathrm{C}(63)$ & $\mathrm{C}(68)$ & $1.41(2)$ \\
\hline$C(64)$ & $C(65)$ & $1.40(2)$ & $\mathrm{C}(65)$ & $C(66)$ & $1.38(2)$ \\
\hline$C(66)$ & $C(67)$ & $1.36(2)$ & $\mathrm{C}(67)$ & $C(68)$ & $1.40(2)$ \\
\hline $\mathrm{C}(71)$ & $C(72)$ & $1.53(2)$ & $\mathrm{C}(73)$ & $\mathrm{C}(74)$ & $1.51(2)$ \\
\hline$C(75)$ & $C(76)$ & $1.11(4)$ & & & \\
\hline
\end{tabular}


Table 21. Bond Angles $\left({ }^{\circ}\right)$ for $\mathrm{CpCp} * \mathrm{Hf}\left(\mathrm{SnPh}_{3}\right) \mathrm{NMe}_{2} \cdot 0.75\left(\mathrm{C}_{4} \mathrm{H}_{10} \mathrm{O}\right)(\mathbf{8})$

\begin{tabular}{|c|c|c|c|c|c|c|c|}
\hline atom & atom & atom & angle & atom & atom & atom & angle \\
\hline $\operatorname{Sn}(1)$ & $\operatorname{Hf}(1)$ & $\mathrm{N}(1)$ & $90.6(2)$ & $\operatorname{Sn}(1)$ & $\operatorname{Hf}(1)$ & $\mathrm{C}(1)$ & $73.2(3)$ \\
\hline $\operatorname{Sn}(1)$ & $\mathrm{Hf}(1)$ & $C(2)$ & $91.7(3)$ & $\operatorname{Sn}(1)$ & $\operatorname{Hf}(1)$ & $\mathrm{C}(3)$ & $123.9(2)$ \\
\hline $\operatorname{Sn}(1)$ & $\mathrm{Hf}(1)$ & C(4) & $123.6(2)$ & $\operatorname{Sn}(1)$ & $\operatorname{Hf}(1)$ & $\mathrm{C}(5)$ & $91.0(3)$ \\
\hline $\operatorname{Sn}(1)$ & $\operatorname{Hf}(1)$ & $C(6)$ & $87.9(3)$ & $\operatorname{Sn}(1)$ & $\operatorname{Hf}(1)$ & $C(7)$ & $83.0(2)$ \\
\hline $\operatorname{Sn}(1)$ & $\operatorname{Hf}(1)$ & $C(8)$ & $110.4(2)$ & $\operatorname{Sn}(1)$ & $\operatorname{Hf}(1)$ & $\mathrm{C}(9)$ & $137.5(2)$ \\
\hline $\operatorname{Sn}(1)$ & $\operatorname{Hf}(1)$ & $C(10)$ & $119.0(2)$ & $\operatorname{Sn}(1)$ & $\operatorname{Hf}(1)$ & $C(100)$ & $101.12(2)$ \\
\hline $\operatorname{Sn}(1)$ & $\operatorname{Hf}(1)$ & $C(101)$ & $108.61(2)$ & $\mathrm{N}(1)$ & $\operatorname{Hf}(1)$ & $\mathrm{C}(1)$ & $106.4(3)$ \\
\hline $\mathrm{N}(1)$ & $\operatorname{Hf}(1)$ & $C(2)$ & $80.1(4)$ & $\mathrm{N}(1)$ & $\operatorname{Hf}(1)$ & $C(3)$ & $87.6(4)$ \\
\hline $\mathrm{N}(1)$ & $\mathrm{Hf}(1)$ & $\mathrm{C}(4)$ & $120.1(3)$ & $\mathrm{N}(1)$ & $\operatorname{Hf}(1)$ & $\mathrm{C}(5)$ & $133.9(4)$ \\
\hline $\mathrm{N}(1)$ & $\operatorname{Hf}(1)$ & $C(6)$ & $94.6(4)$ & $\mathrm{N}(1)$ & $\operatorname{Hf}(1)$ & $C(7)$ & $127.0(3)$ \\
\hline $\mathrm{N}(1)$ & $\operatorname{Hf}(1)$ & $\mathrm{C}(8)$ & $139.4(3)$ & $\mathrm{N}(1)$ & $\operatorname{Hf}(1)$ & $\mathrm{C}(9)$ & $109.6(4)$ \\
\hline $\mathrm{N}(1)$ & $\operatorname{Hf}(1)$ & $C(10)$ & $85.4(4)$ & $\mathrm{N}(1)$ & $\mathrm{Hf}(1)$ & $C(100)$ & $106.8(2)$ \\
\hline $\mathrm{N}(1)$ & $\operatorname{Hf}(1)$ & $C(101)$ & $112.6(2)$ & $C(1)$ & $\operatorname{Hf}(1)$ & $\mathrm{C}(2)$ & $32.3(4)$ \\
\hline C(1) & $\mathrm{Hf}(1)$ & $C(3)$ & $54.0(4)$ & $\mathrm{C}(1)$ & $\operatorname{Hf}(1)$ & $C(4)$ & $54.1(3)$ \\
\hline $\mathrm{C}(1)$ & $\operatorname{Hf}(1)$ & $C(5)$ & $32.1(3)$ & $C(1)$ & $\operatorname{Hf}(1)$ & $C(6)$ & $151.5(4)$ \\
\hline C(1) & $\operatorname{Hf}(1)$ & $C(7)$ & $121.4(4)$ & $C(1)$ & $\operatorname{Hf}(1)$ & $\mathrm{C}(8)$ & $112.8(3)$ \\
\hline $\mathrm{C}(1)$ & $\mathrm{Hf}(1)$ & $\mathrm{C}(9)$ & $130.6(4)$ & $\mathrm{C}(1)$ & $\operatorname{Hf}(1)$ & $\mathrm{C}(10)$ & $163.6(3)$ \\
\hline C(1) & $\operatorname{Hf}(1)$ & $C(100)$ & 27.9(3) & $C(1)$ & $\operatorname{Hf}(1)$ & $C(101)$ & $140.9(3)$ \\
\hline $\mathrm{C}(2)$ & $\mathrm{Hf}(1)$ & $C(3)$ & $33.0(3)$ & $C(2)$ & $\operatorname{Hf}(1)$ & $C(4)$ & $54.6(4)$ \\
\hline$C(2)$ & $\mathrm{Hf}(1)$ & $C(5)$ & $53.8(4)$ & $C(2)$ & $\mathrm{Hf}(1)$ & $C(6)$ & $174.7(4)$ \\
\hline $\mathrm{C}(2)$ & $\operatorname{Hf}(1)$ & $C(7)$ & $152.3(4)$ & $C(2)$ & $\operatorname{Hf}(1)$ & $\mathrm{C}(8)$ & 131.1(3) \\
\hline$C(2)$ & $\operatorname{Hf}(1)$ & $\mathrm{C}(9)$ & $127.7(4)$ & $C(2)$ & $\operatorname{Hf}(1)$ & $\mathrm{C}(10)$ & $146.2(4)$ \\
\hline $\mathrm{C}(2)$ & $\operatorname{Hf}(1)$ & $C(100)$ & $28.6(3)$ & $C(2)$ & $\operatorname{Hf}(1)$ & $C(101)$ & $155.4(3)$ \\
\hline C(3) & $\mathrm{Hf}(1)$ & $C(4)$ & $33.1(3)$ & $C(3)$ & $\operatorname{Hf}(1)$ & $\mathrm{C}(5)$ & $54.4(4)$ \\
\hline C(3) & $\operatorname{Hf}(1)$ & $C(6)$ & 148.1(4) & $C(3)$ & $\operatorname{Hf}(1)$ & $C(7)$ & 138.1(4) \\
\hline C(3) & $\operatorname{Hf}(1)$ & $C(8)$ & $106.6(3)$ & $C(3)$ & $\operatorname{Hf}(1)$ & $\mathrm{C}(9)$ & $94.8(3)$ \\
\hline C(3) & $\operatorname{Hf}(1)$ & $C(10)$ & $116.7(4)$ & $C(3)$ & $\operatorname{Hf}(1)$ & $C(100)$ & $29.1(2)$ \\
\hline C(3) & $\mathrm{Hf}(1)$ & $C(101)$ & $123.6(2)$ & $\mathrm{C}(4)$ & $\operatorname{Hf}(1)$ & $C(5)$ & $33.1(3)$ \\
\hline C(4) & $\mathrm{Hf}(1)$ & $C(6)$ & $129.6(4)$ & $\mathrm{C}(4)$ & $\operatorname{Hf}(1)$ & $C(7)$ & $106.5(3)$ \\
\hline C(4) & $\mathrm{Hf}(1)$ & $C(8)$ & $77.3(3)$ & $\mathrm{C}(4)$ & $\operatorname{Hf}(1)$ & $\mathrm{C}(9)$ & 78.7(4) \\
\hline $\mathrm{C}(4)$ & $\mathrm{Hf}(1)$ & $C(10)$ & $110.2(3)$ & $C(4)$ & $\operatorname{Hf}(1)$ & $C(100)$ & $29.1(2)$ \\
\hline C(4) & $\operatorname{Hf}(1)$ & $C(101)$ & 101.4(2) & $C(5)$ & $\operatorname{Hf}(1)$ & $C(6)$ & 131.4(4) \\
\hline C(5) & $\mathrm{Hf}(1)$ & $C(7)$ & 98.9(4) & $\mathrm{C}(5)$ & $\operatorname{Hf}(1)$ & $\mathrm{C}(8)$ & $81.8(3)$ \\
\hline C(5) & $\operatorname{Hf}(1)$ & $\mathrm{C}(9)$ & $99.7(4)$ & $C(5)$ & $\operatorname{Hf}(1)$ & $\mathrm{C}(10)$ & $132.1(4)$ \\
\hline C(5) & $\mathrm{Hf}(1)$ & $C(100)$ & $28.4(3)$ & $\mathrm{C}(5)$ & $\operatorname{Hf}(1)$ & $C(101)$ & $110.3(3)$ \\
\hline$C(6)$ & $\operatorname{Hf}(1)$ & $C(7)$ & $32.8(3)$ & $C(6)$ & $\operatorname{Hf}(1)$ & $\mathrm{C}(8)$ & $53.7(3)$ \\
\hline C(6) & $\mathrm{Hf}(1)$ & $\mathrm{C}(9)$ & $54.5(3)$ & $C(6)$ & $\operatorname{Hf}(1)$ & $\mathrm{C}(10)$ & $32.5(3)$ \\
\hline$C(6)$ & $\operatorname{Hf}(1)$ & $C(100)$ & $156.5(3)$ & $C(6)$ & $\operatorname{Hf}(1)$ & $C(101)$ & $28.2(3)$ \\
\hline $\mathrm{C}(7)$ & $\operatorname{Hf}(1)$ & $\mathrm{C}(8)$ & $32.2(3)$ & $C(7)$ & $\operatorname{Hf}(1)$ & $\mathrm{C}(9)$ & $54.7(3)$ \\
\hline $\mathrm{C}(7)$ & $\operatorname{Hf}(1)$ & $C(10)$ & $54.3(4)$ & $C(7)$ & $\operatorname{Hf}(1)$ & $C(100)$ & $126.1(2)$ \\
\hline$C(7)$ & $\operatorname{Hf}(1)$ & $C(101)$ & $28.6(2)$ & $C(8)$ & $\operatorname{Hf}(1)$ & $\mathrm{C}(9)$ & $33.3(3)$ \\
\hline \multirow[t]{2}{*}{$\mathrm{C}(8)$} & $\mathrm{Hf}(1)$ & $C(10)$ & $54.1(3)$ & $C(8)$ & $\operatorname{Hf}(1)$ & $C(100)$ & $102.8(2)$ \\
\hline & & & & S28 & & & \\
\hline
\end{tabular}




\begin{tabular}{|c|c|c|c|c|c|c|c|}
\hline$C(8)$ & $\operatorname{Hf}(1)$ & $\mathrm{C}(101)$ & $28.5(2)$ & $\mathrm{C}(9)$ & $\mathrm{Hf}(1)$ & $\mathrm{C}(10)$ & $32.9(3)$ \\
\hline$C(9)$ & $\operatorname{Hf}(1)$ & $\mathrm{C}(100)$ & $107.7(3)$ & $\mathrm{C}(9)$ & $\operatorname{Hf}(1)$ & $\mathrm{C}(101)$ & $29.3(2)$ \\
\hline$C(10)$ & $\operatorname{Hf}(1)$ & $\mathrm{C}(100)$ & $138.1(2)$ & $\mathrm{C}(10)$ & $\operatorname{Hf}(1)$ & $\mathrm{C}(101)$ & $28.5(3)$ \\
\hline$C(100)$ & $\operatorname{Hf}(1)$ & $\mathrm{C}(101)$ & $129.48(2)$ & $\operatorname{Sn}(2)$ & $\operatorname{Hf}(2)$ & $\mathrm{N}(2)$ & $89.7(3)$ \\
\hline $\operatorname{Sn}(2)$ & $\operatorname{Hf}(2)$ & $C(36)$ & $71.6(3)$ & $\operatorname{Sn}(2)$ & $\operatorname{Hf}(2)$ & $\mathrm{C}(37)$ & $88.5(3)$ \\
\hline $\operatorname{Sn}(2)$ & $\operatorname{Hf}(2)$ & $\mathrm{C}(38)$ & $120.9(3)$ & $\operatorname{Sn}(2)$ & $\operatorname{Hf}(2)$ & $\mathrm{C}(39)$ & $123.5(3)$ \\
\hline $\operatorname{Sn}(2)$ & $\operatorname{Hf}(2)$ & $\mathrm{C}(40)$ & $91.9(3)$ & $\operatorname{Sn}(2)$ & $\operatorname{Hf}(2)$ & $\mathrm{C}(41)$ & $88.8(2)$ \\
\hline $\operatorname{Sn}(2)$ & $\operatorname{Hf}(2)$ & $C(42)$ & 119.1(3) & $\operatorname{Sn}(2)$ & $\operatorname{Hf}(2)$ & $\mathrm{C}(43)$ & $138.5(3)$ \\
\hline $\operatorname{Sn}(2)$ & $\operatorname{Hf}(2)$ & $\mathrm{C}(44)$ & $112.4(3)$ & $\operatorname{Sn}(2)$ & $\operatorname{Hf}(2)$ & $\mathrm{C}(45)$ & $84.8(2)$ \\
\hline $\operatorname{Sn}(2)$ & $\operatorname{Hf}(2)$ & $\mathrm{C}(102)$ & $99.68(2)$ & $\operatorname{Sn}(2)$ & $\operatorname{Hf}(2)$ & $C(103)$ & $109.95(2)$ \\
\hline $\mathrm{N}(2)$ & $\operatorname{Hf}(2)$ & $C(36)$ & $108.1(4)$ & $\mathrm{N}(2)$ & $\operatorname{Hf}(2)$ & $\mathrm{C}(37)$ & $80.9(4)$ \\
\hline $\mathrm{N}(2)$ & $\operatorname{Hf}(2)$ & $\mathrm{C}(38)$ & $86.6(4)$ & $\mathrm{N}(2)$ & $\operatorname{Hf}(2)$ & $\mathrm{C}(39)$ & $119.1(4)$ \\
\hline $\mathrm{N}(2)$ & $\operatorname{Hf}(2)$ & $\mathrm{C}(40)$ & $135.8(4)$ & $\mathrm{N}(2)$ & $\operatorname{Hf}(2)$ & $\mathrm{C}(41)$ & $95.3(4)$ \\
\hline $\mathrm{N}(2)$ & $\operatorname{Hf}(2)$ & $\mathrm{C}(42)$ & $85.0(4)$ & $\mathrm{N}(2)$ & $\operatorname{Hf}(2)$ & $\mathrm{C}(43)$ & $110.0(4)$ \\
\hline $\mathrm{N}(2)$ & $\operatorname{Hf}(2)$ & $\mathrm{C}(44)$ & $138.8(4)$ & $\mathrm{N}(2)$ & $\operatorname{Hf}(2)$ & $C(45)$ & $126.9(4)$ \\
\hline $\mathrm{N}(2)$ & $\operatorname{Hf}(2)$ & $\mathrm{C}(102)$ & $106.5(3)$ & $\mathrm{N}(2)$ & $\operatorname{Hf}(2)$ & $C(103)$ & $113.0(3)$ \\
\hline$C(36)$ & $\operatorname{Hf}(2)$ & $C(37)$ & $32.5(4)$ & $C(36)$ & $\operatorname{Hf}(2)$ & $\mathrm{C}(38)$ & $54.2(4)$ \\
\hline$C(36)$ & $\operatorname{Hf}(2)$ & $\mathrm{C}(39)$ & $54.2(4)$ & $C(36)$ & $\operatorname{Hf}(2)$ & $\mathrm{C}(40)$ & $33.2(4)$ \\
\hline$C(36)$ & $\operatorname{Hf}(2)$ & $\mathrm{C}(41)$ & $148.9(4)$ & $C(36)$ & $\operatorname{Hf}(2)$ & $\mathrm{C}(42)$ & $163.8(4)$ \\
\hline$C(36)$ & $\operatorname{Hf}(2)$ & $\mathrm{C}(43)$ & $129.9(4)$ & $C(36)$ & $\operatorname{Hf}(2)$ & $\mathrm{C}(44)$ & $111.7(4)$ \\
\hline$C(36)$ & $\operatorname{Hf}(2)$ & $C(45)$ & $119.7(4)$ & $C(36)$ & $\operatorname{Hf}(2)$ & $C(102)$ & $28.2(3)$ \\
\hline$C(36)$ & $\operatorname{Hf}(2)$ & $\mathrm{C}(103)$ & $138.8(3)$ & $C(37)$ & $\operatorname{Hf}(2)$ & $\mathrm{C}(38)$ & $32.7(4)$ \\
\hline$C(37)$ & $\operatorname{Hf}(2)$ & $\mathrm{C}(39)$ & $54.5(5)$ & $\mathrm{C}(37)$ & $\operatorname{Hf}(2)$ & $\mathrm{C}(40)$ & $55.0(5)$ \\
\hline$C(37)$ & $\operatorname{Hf}(2)$ & $\mathrm{C}(41)$ & $175.4(4)$ & $\mathrm{C}(37)$ & $\operatorname{Hf}(2)$ & $\mathrm{C}(42)$ & $149.0(4)$ \\
\hline C(37) & $\operatorname{Hf}(2)$ & $\mathrm{C}(43)$ & $129.4(4)$ & $\mathrm{C}(37)$ & $\operatorname{Hf}(2)$ & $\mathrm{C}(44)$ & $131.5(4)$ \\
\hline C(37) & $\operatorname{Hf}(2)$ & $C(45)$ & $151.2(4)$ & $\mathrm{C}(37)$ & $\operatorname{Hf}(2)$ & $C(102)$ & $28.0(3)$ \\
\hline C(37) & $\operatorname{Hf}(2)$ & $\mathrm{C}(103)$ & $156.2(3)$ & $\mathrm{C}(38)$ & $\operatorname{Hf}(2)$ & $\mathrm{C}(39)$ & $33.4(4)$ \\
\hline $\mathrm{C}(38)$ & $\operatorname{Hf}(2)$ & $\mathrm{C}(40)$ & $55.4(4)$ & $\mathrm{C}(38)$ & $\operatorname{Hf}(2)$ & $\mathrm{C}(41)$ & $150.3(4)$ \\
\hline $\mathrm{C}(38)$ & $\operatorname{Hf}(2)$ & $\mathrm{C}(42)$ & $119.3(4)$ & $\mathrm{C}(38)$ & $\operatorname{Hf}(2)$ & $\mathrm{C}(43)$ & $97.1(4)$ \\
\hline $\mathrm{C}(38)$ & $\operatorname{Hf}(2)$ & $\mathrm{C}(44)$ & $108.3(5)$ & $\mathrm{C}(38)$ & $\operatorname{Hf}(2)$ & $\mathrm{C}(45)$ & $139.8(4)$ \\
\hline $\mathrm{C}(38)$ & $\operatorname{Hf}(2)$ & $\mathrm{C}(102)$ & $28.8(3)$ & $\mathrm{C}(38)$ & $\operatorname{Hf}(2)$ & $C(103)$ & $125.5(3)$ \\
\hline C(39) & $\operatorname{Hf}(2)$ & $\mathrm{C}(40)$ & $33.0(4)$ & $C(39)$ & $\operatorname{Hf}(2)$ & $\mathrm{C}(41)$ & $130.0(4)$ \\
\hline C(39) & $\operatorname{Hf}(2)$ & $C(42)$ & $111.5(4)$ & $C(39)$ & $\operatorname{Hf}(2)$ & $\mathrm{C}(43)$ & $79.1(4)$ \\
\hline C(39) & $\operatorname{Hf}(2)$ & $\mathrm{C}(44)$ & $78.2(4)$ & $C(39)$ & $\operatorname{Hf}(2)$ & $C(45)$ & $107.4(4)$ \\
\hline C(39) & $\operatorname{Hf}(2)$ & $\mathrm{C}(102)$ & $29.4(3)$ & $C(39)$ & $\operatorname{Hf}(2)$ & $C(103)$ & $101.8(3)$ \\
\hline$C(40)$ & $\operatorname{Hf}(2)$ & $\mathrm{C}(41)$ & $128.8(4)$ & $C(40)$ & $\operatorname{Hf}(2)$ & $\mathrm{C}(42)$ & $130.7(4)$ \\
\hline$C(40)$ & $\operatorname{Hf}(2)$ & $\mathrm{C}(43)$ & $97.4(4)$ & $C(40)$ & $\operatorname{Hf}(2)$ & $\mathrm{C}(44)$ & $79.9(5)$ \\
\hline $\mathrm{C}(40)$ & $\operatorname{Hf}(2)$ & $C(45)$ & $97.2(4)$ & $C(40)$ & $\operatorname{Hf}(2)$ & $C(102)$ & $30.0(3)$ \\
\hline $\mathrm{C}(40)$ & $\operatorname{Hf}(2)$ & $\mathrm{C}(103)$ & $107.7(4)$ & $\mathrm{C}(41)$ & $\operatorname{Hf}(2)$ & $\mathrm{C}(42)$ & $32.0(3)$ \\
\hline $\mathrm{C}(41)$ & $\operatorname{Hf}(2)$ & $\mathrm{C}(43)$ & $54.3(4)$ & $\mathrm{C}(41)$ & $\operatorname{Hf}(2)$ & $\mathrm{C}(44)$ & $53.1(4)$ \\
\hline $\mathrm{C}(41)$ & $\operatorname{Hf}(2)$ & $C(45)$ & $32.0(3)$ & $\mathrm{C}(41)$ & $\operatorname{Hf}(2)$ & $\mathrm{C}(102)$ & $156.5(2)$ \\
\hline$C(41)$ & $\operatorname{Hf}(2)$ & $\mathrm{C}(103)$ & $28.3(2)$ & $C(42)$ & $\operatorname{Hf}(2)$ & $\mathrm{C}(43)$ & $34.0(4)$ \\
\hline$C(42)$ & $\operatorname{Hf}(2)$ & $\mathrm{C}(44)$ & $53.9(4)$ & $C(42)$ & $\operatorname{Hf}(2)$ & $\mathrm{C}(45)$ & $53.6(4)$ \\
\hline $\mathrm{C}(42)$ & $\operatorname{Hf}(2)$ & $\mathrm{C}(102)$ & $139.9(3)$ & $C(42)$ & $\operatorname{Hf}(2)$ & $C(103)$ & $28.9(3)$ \\
\hline$C(43)$ & $\operatorname{Hf}(2)$ & $\mathrm{C}(44)$ & $32.1(4)$ & $C(43)$ & $\operatorname{Hf}(2)$ & $\mathrm{C}(45)$ & $54.0(4)$ \\
\hline$C(43)$ & $\operatorname{Hf}(2)$ & $\mathrm{C}(102)$ & $108.4(3)$ & $\mathrm{C}(43)$ & $\operatorname{Hf}(2)$ & $\mathrm{C}(103)$ & $29.0(3)$ \\
\hline
\end{tabular}




\begin{tabular}{|c|c|c|c|c|c|c|c|}
\hline $\mathrm{C}(44)$ & $\operatorname{Hf}(2)$ & $C(45)$ & $32.3(4)$ & $C(44)$ & $\operatorname{Hf}(2)$ & $C(102)$ & $103.5(3)$ \\
\hline$C(44)$ & $\operatorname{Hf}(2)$ & $\mathrm{C}(103)$ & $27.8(3)$ & $C(45)$ & $\operatorname{Hf}(2)$ & $C(102)$ & $126.5(3)$ \\
\hline$C(45)$ & $\operatorname{Hf}(2)$ & $\mathrm{C}(103)$ & $28.1(2)$ & $\mathrm{C}(102)$ & $\operatorname{Hf}(2)$ & $\mathrm{C}(103)$ & $129.79(2)$ \\
\hline $\mathrm{Hf}(1)$ & $\operatorname{Sn}(1)$ & $C(16)$ & $123.8(3)$ & $\operatorname{Hf}(1)$ & $\operatorname{Sn}(1)$ & $C(22)$ & $119.7(3)$ \\
\hline $\mathrm{Hf}(1)$ & $\operatorname{Sn}(1)$ & $\mathrm{C}(28)$ & $108.5(3)$ & $C(16)$ & $\operatorname{Sn}(1)$ & $C(22)$ & $100.2(4)$ \\
\hline$C(16)$ & $\operatorname{Sn}(1)$ & $\mathrm{C}(28)$ & $100.0(4)$ & $\mathrm{C}(22)$ & $\operatorname{Sn}(1)$ & $\mathrm{C}(28)$ & $100.9(4)$ \\
\hline $\mathrm{Hf}(2)$ & $\operatorname{Sn}(2)$ & $\mathrm{C}(51)$ & $122.9(3)$ & $\operatorname{Hf}(2)$ & $\operatorname{Sn}(2)$ & $C(57)$ & $120.3(3)$ \\
\hline $\mathrm{Hf}(2)$ & $\operatorname{Sn}(2)$ & $C(63)$ & $109.8(3)$ & $\mathrm{C}(51)$ & $\operatorname{Sn}(2)$ & $C(57)$ & $98.2(4)$ \\
\hline $\mathrm{C}(51)$ & $\operatorname{Sn}(2)$ & $C(63)$ & $100.8(4)$ & $C(57)$ & $\operatorname{Sn}(2)$ & $C(63)$ & $101.3(4)$ \\
\hline$C(72)$ & $\mathrm{O}(1)$ & $C(73)$ & $112.4(9)$ & $\mathrm{O}(2)$ & $\mathrm{O}(2)$ & $C(75)$ & $121(2)$ \\
\hline $\mathrm{Hf}(1)$ & $\mathrm{N}(1)$ & $C(34)$ & $134.4(7)$ & $\operatorname{Hf}(1)$ & $\mathrm{N}(1)$ & $C(35)$ & $119.2(7)$ \\
\hline$C(34)$ & $\mathrm{N}(1)$ & $C(35)$ & $106.4(9)$ & $\operatorname{Hf}(2)$ & $\mathrm{N}(2)$ & $\mathrm{C}(69)$ & $132.8(8)$ \\
\hline $\mathrm{Hf}(2)$ & $\mathrm{N}(2)$ & $C(70)$ & $119.7(8)$ & $C(69)$ & $\mathrm{N}(2)$ & $\mathrm{C}(70)$ & $107.5(10)$ \\
\hline $\mathrm{Hf}(1)$ & $\mathrm{C}(1)$ & $\mathrm{C}(2)$ & $72.9(7)$ & $\operatorname{Hf}(1)$ & $\mathrm{C}(1)$ & $C(5)$ & $74.5(7)$ \\
\hline $\mathrm{Hf}(1)$ & $\mathrm{C}(1)$ & $\mathrm{C}(100)$ & $60.5(5)$ & $\mathrm{C}(2)$ & $\mathrm{C}(1)$ & $C(5)$ & $109(1)$ \\
\hline$C(2)$ & $\mathrm{C}(1)$ & $\mathrm{C}(100)$ & $54.4(6)$ & $C(5)$ & $\mathrm{C}(1)$ & $\mathrm{C}(100)$ & $54.9(7)$ \\
\hline $\mathrm{Hf}(1)$ & $C(2)$ & $\mathrm{C}(1)$ & $74.8(7)$ & $\operatorname{Hf}(1)$ & $\mathrm{C}(2)$ & $\mathrm{C}(3)$ & $72.3(6)$ \\
\hline $\mathrm{Hf}(1)$ & $C(2)$ & $\mathrm{C}(100)$ & $61.6(5)$ & $\mathrm{C}(1)$ & $C(2)$ & $\mathrm{C}(3)$ & 107(1) \\
\hline $\mathrm{C}(1)$ & $\mathrm{C}(2)$ & $\mathrm{C}(100)$ & $53.6(7)$ & $\mathrm{C}(3)$ & $\mathrm{C}(2)$ & $\mathrm{C}(100)$ & $54.1(6)$ \\
\hline $\mathrm{Hf}(1)$ & $C(3)$ & $\mathrm{C}(2)$ & $74.7(6)$ & $\operatorname{Hf}(1)$ & $\mathrm{C}(3)$ & $\mathrm{C}(4)$ & $73.9(6)$ \\
\hline $\mathrm{Hf}(1)$ & $\mathrm{C}(3)$ & $\mathrm{C}(100)$ & $62.9(5)$ & $C(2)$ & $\mathrm{C}(3)$ & $\mathrm{C}(4)$ & $108.2(10)$ \\
\hline $\mathrm{C}(2)$ & $C(3)$ & $\mathrm{C}(100)$ & $53.7(6)$ & $\mathrm{C}(4)$ & $\mathrm{C}(3)$ & $\mathrm{C}(100)$ & $54.4(6)$ \\
\hline $\mathrm{Hf}(1)$ & $C(4)$ & $\mathrm{C}(3)$ & $73.0(6)$ & $\operatorname{Hf}(1)$ & $\mathrm{C}(4)$ & $\mathrm{C}(5)$ & $75.7(7)$ \\
\hline $\mathrm{Hf}(1)$ & $\mathrm{C}(4)$ & $\mathrm{C}(100)$ & $62.4(4)$ & $\mathrm{C}(3)$ & $\mathrm{C}(4)$ & $C(5)$ & $107.6(9)$ \\
\hline$C(3)$ & $\mathrm{C}(4)$ & $\mathrm{C}(100)$ & $54.0(6)$ & $C(5)$ & $\mathrm{C}(4)$ & $\mathrm{C}(100)$ & $53.6(6)$ \\
\hline $\mathrm{Hf}(1)$ & $C(5)$ & $\mathrm{C}(1)$ & $73.4(7)$ & $\operatorname{Hf}(1)$ & $\mathrm{C}(5)$ & $\mathrm{C}(4)$ & $71.3(6)$ \\
\hline $\mathrm{Hf}(1)$ & $C(5)$ & $\mathrm{C}(100)$ & $60.0(5)$ & $\mathrm{C}(1)$ & $C(5)$ & $\mathrm{C}(4)$ & $107.2(10)$ \\
\hline$C(1)$ & $\mathrm{C}(5)$ & $\mathrm{C}(100)$ & $53.4(7)$ & $\mathrm{C}(4)$ & $\mathrm{C}(5)$ & $\mathrm{C}(100)$ & $53.7(6)$ \\
\hline $\mathrm{Hf}(1)$ & $C(6)$ & $\mathrm{C}(7)$ & $73.7(6)$ & $\operatorname{Hf}(1)$ & $\mathrm{C}(6)$ & $\mathrm{C}(10)$ & $72.5(6)$ \\
\hline $\mathrm{Hf}(1)$ & $\mathrm{C}(6)$ & $\mathrm{C}(11)$ & $130.6(8)$ & $\operatorname{Hf}(1)$ & $\mathrm{C}(6)$ & $\mathrm{C}(101)$ & $60.2(4)$ \\
\hline$C(7)$ & $\mathrm{C}(6)$ & $\mathrm{C}(10)$ & 107.9(9) & $C(7)$ & $\mathrm{C}(6)$ & $\mathrm{C}(11)$ & $123(1)$ \\
\hline$C(7)$ & $\mathrm{C}(6)$ & $\mathrm{C}(101)$ & $54.1(6)$ & $C(10)$ & $\mathrm{C}(6)$ & $\mathrm{C}(11)$ & $127(1)$ \\
\hline$C(10)$ & $\mathrm{C}(6)$ & $\mathrm{C}(101)$ & $53.8(6)$ & $\mathrm{C}(11)$ & $\mathrm{C}(6)$ & $\mathrm{C}(101)$ & $169(1)$ \\
\hline $\mathrm{Hf}(1)$ & $C(7)$ & $\mathrm{C}(6)$ & $73.5(6)$ & $\operatorname{Hf}(1)$ & $C(7)$ & $\mathrm{C}(8)$ & $72.9(6)$ \\
\hline $\mathrm{Hf}(1)$ & $C(7)$ & $\mathrm{C}(12)$ & $129.3(7)$ & $\operatorname{Hf}(1)$ & $C(7)$ & $\mathrm{C}(101)$ & $60.1(4)$ \\
\hline$C(6)$ & $C(7)$ & $\mathrm{C}(8)$ & $107.2(9)$ & $C(6)$ & $C(7)$ & $\mathrm{C}(12)$ & $124.6(10)$ \\
\hline$C(6)$ & $C(7)$ & $\mathrm{C}(101)$ & $53.1(6)$ & $\mathrm{C}(8)$ & $C(7)$ & $\mathrm{C}(12)$ & $126(1)$ \\
\hline $\mathrm{C}(8)$ & $C(7)$ & $\mathrm{C}(101)$ & $54.1(6)$ & $\mathrm{C}(12)$ & $C(7)$ & $\mathrm{C}(101)$ & $170.4(10)$ \\
\hline $\mathrm{Hf}(1)$ & $\mathrm{C}(8)$ & $\mathrm{C}(7)$ & $74.9(6)$ & $\operatorname{Hf}(1)$ & $\mathrm{C}(8)$ & $\mathrm{C}(9)$ & $70.6(6)$ \\
\hline $\mathrm{Hf}(1)$ & $\mathrm{C}(8)$ & $\mathrm{C}(13)$ & $125.8(7)$ & $\operatorname{Hf}(1)$ & $\mathrm{C}(8)$ & $\mathrm{C}(101)$ & $61.2(4)$ \\
\hline$C(7)$ & $\mathrm{C}(8)$ & $\mathrm{C}(9)$ & $108.6(9)$ & $C(7)$ & $\mathrm{C}(8)$ & $\mathrm{C}(13)$ & $124.9(10)$ \\
\hline$C(7)$ & $\mathrm{C}(8)$ & $\mathrm{C}(101)$ & $55.1(6)$ & $\mathrm{C}(9)$ & $\mathrm{C}(8)$ & $\mathrm{C}(13)$ & $126.1(10)$ \\
\hline$C(9)$ & $\mathrm{C}(8)$ & $\mathrm{C}(101)$ & $53.5(6)$ & $\mathrm{C}(13)$ & $\mathrm{C}(8)$ & $\mathrm{C}(101)$ & $172(1)$ \\
\hline $\mathrm{Hf}(1)$ & $\mathrm{C}(9)$ & $\mathrm{C}(8)$ & $76.1(6)$ & $\operatorname{Hf}(1)$ & $\mathrm{C}(9)$ & $\mathrm{C}(10)$ & $75.9(6)$ \\
\hline $\mathrm{Hf}(1)$ & $\mathrm{C}(9)$ & $\mathrm{C}(14)$ & $122.2(7)$ & $\operatorname{Hf}(1)$ & $\mathrm{C}(9)$ & $\mathrm{C}(101)$ & $64.1(4)$ \\
\hline$C(8)$ & $\mathrm{C}(9)$ & $\mathrm{C}(10)$ & $107.7(9)$ & $\mathrm{C}(8)$ & $\mathrm{C}(9)$ & $\mathrm{C}(14)$ & $126(1)$ \\
\hline
\end{tabular}




\begin{tabular}{|c|c|c|c|c|c|c|c|}
\hline $\mathrm{C}(8)$ & $\mathrm{C}(9)$ & $\mathrm{C}(101)$ & $53.7(6)$ & $C(10)$ & $\mathrm{C}(9)$ & $C(14)$ & $124.8(10)$ \\
\hline$C(10)$ & $\mathrm{C}(9)$ & $\mathrm{C}(101)$ & $54.0(6)$ & $C(14)$ & $\mathrm{C}(9)$ & $\mathrm{C}(101)$ & $173.6(10)$ \\
\hline $\mathrm{Hf}(1)$ & $C(10)$ & $C(6)$ & $75.0(6)$ & $\mathrm{Hf}(1)$ & $\mathrm{C}(10)$ & $\mathrm{C}(9)$ & $71.1(6)$ \\
\hline $\mathrm{Hf}(1)$ & $C(10)$ & $C(15)$ & $123.2(8)$ & $\mathrm{Hf}(1)$ & $\mathrm{C}(10)$ & $\mathrm{C}(101)$ & $61.5(4)$ \\
\hline$C(6)$ & $C(10)$ & $\mathrm{C}(9)$ & 108.3(9) & $C(6)$ & $\mathrm{C}(10)$ & $C(15)$ & $126(1)$ \\
\hline$C(6)$ & $\mathrm{C}(10)$ & $\mathrm{C}(101)$ & $54.1(6)$ & $\mathrm{C}(9)$ & $\mathrm{C}(10)$ & $C(15)$ & $124.6(10)$ \\
\hline $\mathrm{C}(9)$ & $\mathrm{C}(10)$ & $\mathrm{C}(101)$ & $54.2(6)$ & $C(15)$ & $\mathrm{C}(10)$ & $\mathrm{C}(101)$ & $175.1(10)$ \\
\hline $\operatorname{Sn}(1)$ & $C(16)$ & $\mathrm{C}(17)$ & $121.7(9)$ & $\operatorname{Sn}(1)$ & $C(16)$ & $\mathrm{C}(21)$ & $122.3(8)$ \\
\hline$C(17)$ & $C(16)$ & $\mathrm{C}(21)$ & $115(1)$ & $C(16)$ & $\mathrm{C}(17)$ & $\mathrm{C}(18)$ & $122(1)$ \\
\hline$C(17)$ & $\mathrm{C}(18)$ & C(19) & $120(1)$ & $\mathrm{C}(18)$ & $C(19)$ & $C(20)$ & $117(1)$ \\
\hline$C(19)$ & $\mathrm{C}(20)$ & $C(21)$ & 121(1) & $C(16)$ & $\mathrm{C}(21)$ & $C(20)$ & 121(1) \\
\hline $\operatorname{Sn}(1)$ & $\mathrm{C}(22)$ & $\mathrm{C}(23)$ & $120.8(9)$ & $\operatorname{Sn}(1)$ & $\mathrm{C}(22)$ & $C(27)$ & $123.3(8)$ \\
\hline$C(23)$ & $\mathrm{C}(22)$ & $\mathrm{C}(27)$ & $115(1)$ & $C(22)$ & $\mathrm{C}(23)$ & $\mathrm{C}(24)$ & $120(1)$ \\
\hline $\mathrm{C}(23)$ & $\mathrm{C}(24)$ & $C(25)$ & $120(1)$ & $\mathrm{C}(24)$ & $C(25)$ & $C(26)$ & 121(1) \\
\hline$C(25)$ & $C(26)$ & $\mathrm{C}(27)$ & $117(1)$ & $C(22)$ & $\mathrm{C}(27)$ & $C(26)$ & $123(1)$ \\
\hline $\operatorname{Sn}(1)$ & $\mathrm{C}(28)$ & C(29) & $119.2(8)$ & $\operatorname{Sn}(1)$ & $\mathrm{C}(28)$ & $C(33)$ & $121.8(8)$ \\
\hline$C(29)$ & $\mathrm{C}(28)$ & $C(33)$ & $119.0(10)$ & $\mathrm{C}(28)$ & $\mathrm{C}(29)$ & $C(30)$ & $120(1)$ \\
\hline$C(29)$ & $C(30)$ & $\mathrm{C}(31)$ & $118(1)$ & $C(30)$ & $\mathrm{C}(31)$ & $C(32)$ & 121(1) \\
\hline $\mathrm{C}(31)$ & $C(32)$ & $C(33)$ & $120(1)$ & $\mathrm{C}(28)$ & $\mathrm{C}(33)$ & $\mathrm{C}(32)$ & $120(1)$ \\
\hline $\operatorname{Hf}(2)$ & $C(36)$ & $\mathrm{C}(37)$ & $73.8(7)$ & $\operatorname{Hf}(2)$ & $C(36)$ & $\mathrm{C}(40)$ & $74.1(7)$ \\
\hline $\operatorname{Hf}(2)$ & $C(36)$ & $\mathrm{C}(102)$ & $61.6(5)$ & $\mathrm{C}(37)$ & $C(36)$ & $\mathrm{C}(40)$ & $109(1)$ \\
\hline$C(37)$ & $C(36)$ & $C(102)$ & $53.3(8)$ & $\mathrm{C}(40)$ & $C(36)$ & $\mathrm{C}(102)$ & $56.6(8)$ \\
\hline $\mathrm{Hf}(2)$ & $C(37)$ & $C(36)$ & $73.7(7)$ & $\operatorname{Hf}(2)$ & $\mathrm{C}(37)$ & $\mathrm{C}(38)$ & $73.2(7)$ \\
\hline $\operatorname{Hf}(2)$ & $C(37)$ & $\mathrm{C}(102)$ & $61.5(5)$ & $C(36)$ & $C(37)$ & $\mathrm{C}(38)$ & $108(1)$ \\
\hline$C(36)$ & $C(37)$ & $\mathrm{C}(102)$ & $53.7(8)$ & $\mathrm{C}(38)$ & $\mathrm{C}(37)$ & $\mathrm{C}(102)$ & $54.4(8)$ \\
\hline $\mathrm{Hf}(2)$ & $\mathrm{C}(38)$ & $\mathrm{C}(37)$ & $74.1(7)$ & $\operatorname{Hf}(2)$ & $\mathrm{C}(38)$ & $\mathrm{C}(39)$ & $73.2(7)$ \\
\hline $\mathrm{Hf}(2)$ & $\mathrm{C}(38)$ & $\mathrm{C}(102)$ & $62.1(5)$ & $C(37)$ & $\mathrm{C}(38)$ & $C(39)$ & $107(1)$ \\
\hline$C(37)$ & $\mathrm{C}(38)$ & $\mathrm{C}(102)$ & $52.9(8)$ & C(39) & $\mathrm{C}(38)$ & $\mathrm{C}(102)$ & $54.5(8)$ \\
\hline $\mathrm{Hf}(2)$ & $C(39)$ & $\mathrm{C}(38)$ & $73.4(7)$ & $\mathrm{Hf}(2)$ & $\mathrm{C}(39)$ & $\mathrm{C}(40)$ & $74.7(7)$ \\
\hline $\operatorname{Hf}(2)$ & $C(39)$ & $\mathrm{C}(102)$ & $62.1(5)$ & $\mathrm{C}(38)$ & $\mathrm{C}(39)$ & $\mathrm{C}(40)$ & $109(1)$ \\
\hline$C(38)$ & $\mathrm{C}(39)$ & $\mathrm{C}(102)$ & $53.0(8)$ & $\mathrm{C}(40)$ & $\mathrm{C}(39)$ & $\mathrm{C}(102)$ & $56.2(8)$ \\
\hline $\operatorname{Hf}(2)$ & $\mathrm{C}(40)$ & $C(36)$ & $72.7(7)$ & $\operatorname{Hf}(2)$ & $\mathrm{C}(40)$ & C(39) & $72.3(7)$ \\
\hline $\mathrm{Hf}(2)$ & $\mathrm{C}(40)$ & $\mathrm{C}(102)$ & $60.8(5)$ & $C(36)$ & $\mathrm{C}(40)$ & $C(39)$ & $105(1)$ \\
\hline$C(36)$ & $\mathrm{C}(40)$ & $\mathrm{C}(102)$ & $51.6(8)$ & C(39) & $\mathrm{C}(40)$ & $\mathrm{C}(102)$ & $53.7(8)$ \\
\hline $\operatorname{Hf}(2)$ & $\mathrm{C}(41)$ & $\mathrm{C}(42)$ & $70.7(6)$ & $\operatorname{Hf}(2)$ & $\mathrm{C}(41)$ & $C(45)$ & $73.4(6)$ \\
\hline $\mathrm{Hf}(2)$ & $\mathrm{C}(41)$ & $C(46)$ & $128.1(7)$ & $\operatorname{Hf}(2)$ & $\mathrm{C}(41)$ & $\mathrm{C}(103)$ & $59.1(4)$ \\
\hline $\mathrm{C}(42)$ & $\mathrm{C}(41)$ & $C(45)$ & $108.1(10)$ & $C(42)$ & $\mathrm{C}(41)$ & $\mathrm{C}(46)$ & $128.2(10)$ \\
\hline$C(42)$ & $\mathrm{C}(41)$ & $\mathrm{C}(103)$ & $54.3(6)$ & $\mathrm{C}(45)$ & $\mathrm{C}(41)$ & $C(46)$ & $123(1)$ \\
\hline$C(45)$ & $\mathrm{C}(41)$ & $\mathrm{C}(103)$ & $53.8(6)$ & $\mathrm{C}(46)$ & $\mathrm{C}(41)$ & $\mathrm{C}(103)$ & $172.3(10)$ \\
\hline $\mathrm{Hf}(2)$ & $\mathrm{C}(42)$ & $\mathrm{C}(41)$ & $77.2(6)$ & $\operatorname{Hf}(2)$ & $\mathrm{C}(42)$ & $\mathrm{C}(43)$ & $71.9(6)$ \\
\hline $\operatorname{Hf}(2)$ & $\mathrm{C}(42)$ & $\mathrm{C}(47)$ & $124.6(8)$ & $\operatorname{Hf}(2)$ & $\mathrm{C}(42)$ & $\mathrm{C}(103)$ & $62.5(5)$ \\
\hline $\mathrm{C}(41)$ & $\mathrm{C}(42)$ & $C(43)$ & $107(1)$ & $\mathrm{C}(41)$ & $\mathrm{C}(42)$ & $\mathrm{C}(47)$ & $127(1)$ \\
\hline$C(41)$ & $C(42)$ & $\mathrm{C}(103)$ & $55.2(7)$ & $\mathrm{C}(43)$ & $\mathrm{C}(42)$ & $C(47)$ & $123(1)$ \\
\hline$C(43)$ & $\mathrm{C}(42)$ & $\mathrm{C}(103)$ & $52.5(6)$ & $\mathrm{C}(47)$ & $\mathrm{C}(42)$ & $\mathrm{C}(103)$ & 171(1) \\
\hline $\operatorname{Hf}(2)$ & $\mathrm{C}(43)$ & $C(42)$ & $74.1(7)$ & $\mathrm{Hf}(2)$ & $\mathrm{C}(43)$ & $\mathrm{C}(44)$ & $76.8(7)$ \\
\hline $\mathrm{Hf}(2)$ & $\mathrm{C}(43)$ & $\mathrm{C}(48)$ & $123.1(9)$ & $\operatorname{Hf}(2)$ & $\mathrm{C}(43)$ & $\mathrm{C}(103)$ & $63.7(5)$ \\
\hline
\end{tabular}




\begin{tabular}{|c|c|c|c|c|c|c|c|}
\hline$C(42)$ & $\mathrm{C}(43)$ & $\mathrm{C}(44)$ & $107(1)$ & $C(42)$ & $\mathrm{C}(43)$ & $\mathrm{C}(48)$ & $122(1)$ \\
\hline$C(42)$ & $\mathrm{C}(43)$ & $\mathrm{C}(103)$ & $53.2(7)$ & $\mathrm{C}(44)$ & $\mathrm{C}(43)$ & $\mathrm{C}(48)$ & $128(1)$ \\
\hline$C(44)$ & $\mathrm{C}(43)$ & $\mathrm{C}(103)$ & $54.0(7)$ & $\mathrm{C}(48)$ & $C(43)$ & $C(103)$ & $172(1)$ \\
\hline $\mathrm{Hf}(2)$ & $\mathrm{C}(44)$ & $\mathrm{C}(43)$ & $71.1(7)$ & $\operatorname{Hf}(2)$ & $\mathrm{C}(44)$ & $C(45)$ & $74.8(6)$ \\
\hline $\mathrm{Hf}(2)$ & $\mathrm{C}(44)$ & $\mathrm{C}(49)$ & $127.0(9)$ & $\operatorname{Hf}(2)$ & $\mathrm{C}(44)$ & $C(103)$ & $60.7(5)$ \\
\hline$C(43)$ & $\mathrm{C}(44)$ & $\mathrm{C}(45)$ & $109(1)$ & $\mathrm{C}(43)$ & $\mathrm{C}(44)$ & $\mathrm{C}(49)$ & $123(1)$ \\
\hline $\mathrm{C}(43)$ & $\mathrm{C}(44)$ & $\mathrm{C}(103)$ & $54.8(7)$ & $C(45)$ & $\mathrm{C}(44)$ & $\mathrm{C}(49)$ & $126(1)$ \\
\hline$C(45)$ & $\mathrm{C}(44)$ & $\mathrm{C}(103)$ & $54.3(7)$ & $C(49)$ & $\mathrm{C}(44)$ & $C(103)$ & $172(1)$ \\
\hline $\operatorname{Hf}(2)$ & $C(45)$ & $\mathrm{C}(41)$ & $74.7(6)$ & $\operatorname{Hf}(2)$ & $\mathrm{C}(45)$ & $\mathrm{C}(44)$ & $73.0(7)$ \\
\hline $\operatorname{Hf}(2)$ & $C(45)$ & $\mathrm{C}(50)$ & $127.9(7)$ & $\operatorname{Hf}(2)$ & $C(45)$ & $C(103)$ & $59.8(4)$ \\
\hline$C(41)$ & $C(45)$ & $\mathrm{C}(44)$ & $107(1)$ & $\mathrm{C}(41)$ & $\mathrm{C}(45)$ & $\mathrm{C}(50)$ & $124(1)$ \\
\hline $\mathrm{C}(41)$ & $C(45)$ & $\mathrm{C}(103)$ & $54.9(6)$ & $\mathrm{C}(44)$ & $\mathrm{C}(45)$ & $\mathrm{C}(50)$ & $126(1)$ \\
\hline$C(44)$ & $C(45)$ & $\mathrm{C}(103)$ & $52.9(7)$ & $C(50)$ & $C(45)$ & $\mathrm{C}(103)$ & $172.3(10)$ \\
\hline $\operatorname{Sn}(2)$ & $\mathrm{C}(51)$ & $\mathrm{C}(52)$ & $123.3(8)$ & $\operatorname{Sn}(2)$ & $\mathrm{C}(51)$ & $C(56)$ & $120.4(8)$ \\
\hline$C(52)$ & $\mathrm{C}(51)$ & $C(56)$ & $116(1)$ & $\mathrm{C}(51)$ & $C(52)$ & $\mathrm{C}(53)$ & $121(1)$ \\
\hline$C(52)$ & $\mathrm{C}(53)$ & $\mathrm{C}(54)$ & 119(1) & $\mathrm{C}(53)$ & $\mathrm{C}(54)$ & $C(55)$ & $120(1)$ \\
\hline$C(54)$ & $C(55)$ & $C(56)$ & 119(1) & $\mathrm{C}(51)$ & $C(56)$ & $C(55)$ & $122(1)$ \\
\hline $\operatorname{Sn}(2)$ & $C(57)$ & $\mathrm{C}(58)$ & $121.0(8)$ & $\operatorname{Sn}(2)$ & $C(57)$ & $\mathrm{C}(62)$ & $122.5(9)$ \\
\hline $\mathrm{C}(58)$ & $C(57)$ & $C(62)$ & $116(1)$ & $C(57)$ & $\mathrm{C}(58)$ & $\mathrm{C}(59)$ & $123(1)$ \\
\hline $\mathrm{C}(58)$ & $\mathrm{C}(59)$ & $C(60)$ & 119(1) & $\mathrm{C}(59)$ & $\mathrm{C}(60)$ & $\mathrm{C}(61)$ & $119(1)$ \\
\hline$C(60)$ & $C(61)$ & $C(62)$ & 119(1) & $C(57)$ & $C(62)$ & $\mathrm{C}(61)$ & $120(1)$ \\
\hline $\operatorname{Sn}(2)$ & $C(63)$ & $C(64)$ & $123.5(8)$ & $\operatorname{Sn}(2)$ & $C(63)$ & $C(68)$ & $119.4(8)$ \\
\hline$C(64)$ & $\mathrm{C}(63)$ & $\mathrm{C}(68)$ & $117(1)$ & $C(63)$ & $\mathrm{C}(64)$ & $C(65)$ & 121(1) \\
\hline$C(64)$ & $C(65)$ & $C(66)$ & 119(1) & $C(65)$ & $C(66)$ & $\mathrm{C}(67)$ & $120(1)$ \\
\hline $\mathrm{C}(66)$ & $C(67)$ & $\mathrm{C}(68)$ & $120(1)$ & $\mathrm{C}(63)$ & $\mathrm{C}(68)$ & $C(67)$ & $120(1)$ \\
\hline $\mathrm{O}(1)$ & $C(72)$ & $\mathrm{C}(71)$ & $107(1)$ & $\mathrm{O}(1)$ & $C(73)$ & $\mathrm{C}(74)$ & $108(1)$ \\
\hline $\mathrm{O}(2)$ & $C(75)$ & $C(76)$ & $115(3)$ & $\operatorname{Hf}(1)$ & $C(100)$ & $\mathrm{C}(1)$ & $91.5(5)$ \\
\hline $\mathrm{Hf}(1)$ & $C(100)$ & $C(2)$ & $89.8(5)$ & $\operatorname{Hf}(1)$ & $C(100)$ & $\mathrm{C}(3)$ & $88.0(5)$ \\
\hline $\mathrm{Hf}(1)$ & $C(100)$ & $\mathrm{C}(4)$ & $88.5(5)$ & $\operatorname{Hf}(1)$ & $C(100)$ & $C(5)$ & $91.6(5)$ \\
\hline $\mathrm{C}(1)$ & $\mathrm{C}(100)$ & $C(2)$ & $71.9(7)$ & $\mathrm{C}(1)$ & $C(100)$ & $\mathrm{C}(3)$ & $144.2(7)$ \\
\hline $\mathrm{C}(1)$ & $\mathrm{C}(100)$ & $C(4)$ & $144.2(7)$ & $\mathrm{C}(1)$ & $C(100)$ & $\mathrm{C}(5)$ & $71.6(7)$ \\
\hline$C(2)$ & $\mathrm{C}(100)$ & $\mathrm{C}(3)$ & $72.2(7)$ & $\mathrm{C}(2)$ & $C(100)$ & $\mathrm{C}(4)$ & $143.8(7)$ \\
\hline$C(2)$ & $\mathrm{C}(100)$ & $C(5)$ & $143.6(8)$ & $\mathrm{C}(3)$ & $C(100)$ & $\mathrm{C}(4)$ & $71.6(7)$ \\
\hline$C(3)$ & $\mathrm{C}(100)$ & $C(5)$ & $144.2(7)$ & $\mathrm{C}(4)$ & $C(100)$ & $C(5)$ & $72.6(7)$ \\
\hline $\mathrm{Hf}(1)$ & $\mathrm{C}(101)$ & $C(6)$ & $91.6(5)$ & $\operatorname{Hf}(1)$ & $\mathrm{C}(101)$ & $C(7)$ & $91.3(5)$ \\
\hline $\mathrm{Hf}(1)$ & $\mathrm{C}(101)$ & $\mathrm{C}(8)$ & $90.3(5)$ & $\operatorname{Hf}(1)$ & $\mathrm{C}(101)$ & $\mathrm{C}(9)$ & $86.6(5)$ \\
\hline $\mathrm{Hf}(1)$ & $\mathrm{C}(101)$ & $\mathrm{C}(10)$ & $89.9(5)$ & $C(6)$ & $\mathrm{C}(101)$ & $C(7)$ & $72.8(7)$ \\
\hline $\mathrm{C}(6)$ & $\mathrm{C}(101)$ & $\mathrm{C}(8)$ & $143.5(7)$ & $C(6)$ & $\mathrm{C}(101)$ & $\mathrm{C}(9)$ & $143.8(7)$ \\
\hline$C(6)$ & $\mathrm{C}(101)$ & $\mathrm{C}(10)$ & $72.0(7)$ & $C(7)$ & $\mathrm{C}(101)$ & $\mathrm{C}(8)$ & $70.7(7)$ \\
\hline$C(7)$ & $\mathrm{C}(101)$ & $\mathrm{C}(9)$ & $143.4(7)$ & $C(7)$ & $\mathrm{C}(101)$ & $\mathrm{C}(10)$ & $144.8(7)$ \\
\hline $\mathrm{C}(8)$ & $\mathrm{C}(101)$ & $\mathrm{C}(9)$ & $72.7(7)$ & $\mathrm{C}(8)$ & $\mathrm{C}(101)$ & $\mathrm{C}(10)$ & $144.4(7)$ \\
\hline$C(9)$ & $\mathrm{C}(101)$ & $\mathrm{C}(10)$ & $71.8(7)$ & $\operatorname{Hf}(2)$ & $C(102)$ & $C(36)$ & $90.2(5)$ \\
\hline $\operatorname{Hf}(2)$ & $\mathrm{C}(102)$ & $C(37)$ & $90.5(6)$ & $\operatorname{Hf}(2)$ & $C(102)$ & $\mathrm{C}(38)$ & $89.2(6)$ \\
\hline $\mathrm{Hf}(2)$ & $\mathrm{C}(102)$ & $C(39)$ & $88.5(6)$ & $\operatorname{Hf}(2)$ & $\mathrm{C}(102)$ & $\mathrm{C}(40)$ & $89.2(6)$ \\
\hline$C(36)$ & $\mathrm{C}(102)$ & $C(37)$ & $73.0(9)$ & $C(36)$ & $\mathrm{C}(102)$ & $\mathrm{C}(38)$ & $145.6(10)$ \\
\hline$C(36)$ & $\mathrm{C}(102)$ & $C(39)$ & $141.9(10)$ & $C(36)$ & $\mathrm{C}(102)$ & $\mathrm{C}(40)$ & $71.9(9)$ \\
\hline
\end{tabular}




$\begin{array}{llllllll}\mathrm{C}(37) & \mathrm{C}(102) & \mathrm{C}(38) & 72.6(9) & \mathrm{C}(37) & \mathrm{C}(102) & \mathrm{C}(39) & 145.1(10) \\ \mathrm{C}(37) & \mathrm{C}(102) & \mathrm{C}(40) & 144.8(9) & \mathrm{C}(38) & \mathrm{C}(102) & \mathrm{C}(39) & 72.5(9) \\ \mathrm{C}(38) & \mathrm{C}(102) & \mathrm{C}(40) & 142.5(10) & \mathrm{C}(39) & \mathrm{C}(102) & \mathrm{C}(40) & 70.1(9) \\ \mathrm{Hf}(2) & \mathrm{C}(103) & \mathrm{C}(41) & 92.6(5) & \mathrm{Hf}(2) & \mathrm{C}(103) & \mathrm{C}(42) & 88.6(5) \\ \mathrm{Hf}(2) & \mathrm{C}(103) & \mathrm{C}(43) & 87.3(6) & \mathrm{Hf}(2) & \mathrm{C}(103) & \mathrm{C}(44) & 91.5(6) \\ \mathrm{Hf}(2) & \mathrm{C}(103) & \mathrm{C}(45) & 92.2(5) & \mathrm{C}(41) & \mathrm{C}(103) & \mathrm{C}(42) & 70.5(7) \\ \mathrm{C}(41) & \mathrm{C}(103) & \mathrm{C}(43) & 144.8(7) & \mathrm{C}(41) & \mathrm{C}(103) & \mathrm{C}(44) & 144.0(8) \\ \mathrm{C}(41) & \mathrm{C}(103) & \mathrm{C}(45) & 71.3(7) & \mathrm{C}(42) & \mathrm{C}(103) & \mathrm{C}(43) & 74.2(8) \\ \mathrm{C}(42) & \mathrm{C}(103) & \mathrm{C}(44) & 145.4(8) & \mathrm{C}(42) & \mathrm{C}(103) & \mathrm{C}(45) & 141.9(8) \\ \mathrm{C}(43) & \mathrm{C}(103) & \mathrm{C}(44) & 71.2(8) & \mathrm{C}(43) & \mathrm{C}(103) & \mathrm{C}(45) & 143.9(8) \\ \mathrm{C}(44) & \mathrm{C}(103) & \mathrm{C}(45) & 72.7(8) & & & & \end{array}$

Table 22. Crystal Data for $\mathrm{CpCp} * \mathrm{Hf}\left(\mathrm{SnPh}_{3}\right) \mathrm{Me} \bullet 0.5\left(\mathrm{C}_{5} \mathrm{H}_{12}\right)(\mathbf{9})$

\begin{tabular}{|c|c|}
\hline \multicolumn{2}{|l|}{ Empirical Formula } \\
\hline & 779.93 \\
\hline Crystal Color, Habit & plates, yellow \\
\hline Crystal Dimensions & $0.18 \times 0.14 \times 0.10 \mathrm{~mm}$ \\
\hline Crystal System & monoclinic \\
\hline Lattice Type & Primitive \\
\hline No. of Reflections Used for Unit & \\
\hline Cell Determination ( $2 \theta$ range) & $6312\left(3.0-51.0^{\mathrm{O}}\right)$ \\
\hline Lattice Parameters & $\mathrm{a}=12.3051 \AA$ \\
\hline & $\mathrm{b}=20.3429 \AA$ \\
\hline & $c=13.1785 \AA$ \\
\hline & $\beta=100.6230^{\circ}$ \\
\hline & $\mathrm{V}=3242.32251(10) \AA^{3}$ \\
\hline Space Group & $\mathrm{P} 21_{1} / \mathrm{n}(\# 14)$ \\
\hline$Z$ value & 4 \\
\hline$D_{\text {calc }}$ & $1.598 \mathrm{~g} / \mathrm{cm}^{3}$ \\
\hline $\mathrm{F}_{000}$ & 1540.00 \\
\hline$\mu(\operatorname{MoK} \alpha)$ & $39.86 \mathrm{~cm}^{-1}$ \\
\hline
\end{tabular}


Table 23. Intensity Measurements for $\mathrm{CpCp} * \mathrm{Hf}\left(\mathrm{SnPh}_{3}\right) \mathrm{Me} \bullet 0.5\left(\mathrm{C}_{5} \mathrm{H}_{12}\right)(\mathbf{9})$

Diffractometer

Radiation

Crystal to Detector Distance

Temperature

Scan Type

Scan Rate

$2 \theta_{\max }$

No. of Reflections Measured

Corrections
SMART

$\operatorname{MoK} \alpha(1=0.71069 \AA)$

graphite monochromated

$60.0 \mathrm{~mm}$

$-149{ }^{\circ} \mathrm{C}$

$\omega\left(0.3^{\circ}\right.$ per frame $)$

10.0 seconds per frame

$51.0^{\mathrm{O}}$

Total: 14682

Unique: $4644\left(\mathrm{R}_{\mathrm{int}}=0.053\right)$

Lorentz-polarization Absorption

$(\operatorname{Tmax}=0.685, \mathrm{Tmin}=0.494)$

Table 24. Structure Solution and Refinement for $\mathrm{CpCp} * \mathrm{Hf}\left(\mathrm{SnPh}_{3}\right) \mathrm{Me} \bullet 0.5\left(\mathrm{C}_{5} \mathrm{H}_{12}\right)(9)$

Structure Solution

Refinement

Function Minimized

Least Squares Weights

p-factor

Anomalous Dispersion

No. Observations (I>3.00\%(I))

No. Variables

Reflection/Parameter Ratio

Residuals: R; Rw; Rall

Goodness of Fit Indicator

Max Shift/Error in Final Cycle

Maximum peak in Final Diff. Map

Minimum peak in Final Diff. Map
Direct Methods (SIR92)

Full-matrix least-squares

$\Sigma \omega(|\mathrm{Fol}-| \mathrm{Fcl})^{2}$

$1 / \mathrm{\sigma}^{2}(\mathrm{Fo})=4 \mathrm{Fo}^{2} / \mathrm{\sigma}^{2}\left(\mathrm{Fo}^{2}\right)$

0.030

All non-hydrogen atoms

3724

337

11.05

$0.033 ; 0.039 ; 0.053$

0.94

0.00

$0.72 \mathrm{e}^{-} / \AA^{3}$

$-1.59 \mathrm{e}^{-} / \AA^{3}$

Table 25. Atomic coordinates and $\mathrm{B}_{\mathrm{iso}} / \mathrm{B}_{\text {eq }}$ for $\mathrm{CpCp} * \mathrm{Hf}\left(\mathrm{SnPh}_{3}\right) \mathrm{Me} \bullet 0.5\left(\mathrm{C}_{5} \mathrm{H}_{12}\right)(\mathbf{9})$

$\begin{array}{lllll}\text { atom } & \mathrm{x} & \mathrm{y} & \mathrm{z} & \mathrm{B}_{\mathrm{eq}} \\ \mathrm{Hf}(1) & 0.95458(2) & 0.20838(1) & 0.53490(2) & 1.580(6) \\ \mathrm{Sn}(1) & 0.84420(4) & 0.21756(2) & 0.71706(3) & 1.687(10) \\ \mathrm{C}(1) & 1.0751(7) & 0.2795(5) & 0.6659(8) & 3.6(2) \\ \mathrm{C}(2) & 1.0370(8) & 0.3199(4) & 0.5789(9) & 3.9(2) \\ \mathrm{C}(3) & 1.0838(8) & 0.2954(5) & 0.4952(8) & 4.6(2) \\ \mathrm{C}(4) & 1.1489(6) & 0.2397(4) & 0.5318(6) & 2.5(2) \\ \mathrm{C}(5) & 1.1423(6) & 0.2299(4) & 0.6373(7) & 3.0(2) \\ \mathrm{C}(6) & 0.8357(6) & 0.1097(4) & 0.4741(6) & 2.1(2) \\ \mathrm{C}(7) & 0.8870(6) & 0.1294(4) & 0.3904(5) & 2.2(1) \\ & & & \mathrm{S} 34 & \end{array}$




\begin{tabular}{|c|c|c|c|c|}
\hline $\mathrm{C}(8)$ & $1.0023(6)$ & $0.1215(4)$ & $0.4193(5)$ & $1.9(1)$ \\
\hline$C(9)$ & $1.0228(6)$ & $0.0947(4)$ & $0.5209(5)$ & $1.7(1)$ \\
\hline$C(10)$ & $0.9214(6)$ & $0.0872(3)$ & $0.5547(5)$ & $1.9(1)$ \\
\hline $\mathrm{C}(11)$ & $0.7132(7)$ & $0.1047(5)$ & $0.4714(6)$ & $3.3(2)$ \\
\hline$C(12)$ & $0.8258(7)$ & $0.1489(5)$ & $0.2835(6)$ & $3.1(2)$ \\
\hline$C(13)$ & $1.0844(6)$ & $0.1315(4)$ & $0.3473(5)$ & $2.1(2)$ \\
\hline$C(14)$ & $1.1327(6)$ & $0.0688(4)$ & $0.5785(6)$ & $2.7(2)$ \\
\hline$C(15)$ & $0.9039(7)$ & $0.0507(4)$ & $0.6494(6)$ & $2.5(2)$ \\
\hline$C(16)$ & $0.8254(8)$ & $0.2704(4)$ & $0.4373(6)$ & $3.0(2)$ \\
\hline$C(17)$ & $0.9210(6)$ & $0.1648(4)$ & $0.8558(5)$ & $1.7(1)$ \\
\hline $\mathrm{C}(18)$ & $1.0312(6)$ & $0.1464(4)$ & $0.8719(5)$ & $2.7(2)$ \\
\hline C(19) & $1.0781(7)$ & $0.1083(4)$ & $0.9585(6)$ & $3.3(2)$ \\
\hline$C(20)$ & $1.0181(8)$ & $0.0894(4)$ & $1.0295(6)$ & $3.0(2)$ \\
\hline $\mathrm{C}(21)$ & $0.9088(7)$ & $0.1079(4)$ & $1.0163(6)$ & $2.8(2)$ \\
\hline $\mathrm{C}(22)$ & $0.8600(6)$ & $0.1458(4)$ & $0.9311(6)$ & $2.5(2)$ \\
\hline $\mathrm{C}(23)$ & $0.6712(6)$ & $0.1916(3)$ & $0.7028(5)$ & $1.6(1)$ \\
\hline$C(24)$ & $0.6349(6)$ & $0.1311(4)$ & $0.7353(5)$ & $2.1(2)$ \\
\hline$C(25)$ & $0.5231(7)$ & $0.1156(4)$ & $0.7220(6)$ & $2.5(2)$ \\
\hline$C(26)$ & $0.4435(6)$ & $0.1599(5)$ & $0.6754(6)$ & $2.9(2)$ \\
\hline$C(27)$ & $0.4758(7)$ & $0.2201(4)$ & $0.6422(6)$ & $2.8(2)$ \\
\hline$C(28)$ & $0.5879(6)$ & $0.2351(4)$ & $0.6546(6)$ & $2.3(2)$ \\
\hline C(29) & $0.8475(6)$ & $0.3191(4)$ & $0.7724(5)$ & $1.7(1)$ \\
\hline$C(30)$ & $0.8994(6)$ & $0.3344(4)$ & $0.8739(6)$ & $2.2(2)$ \\
\hline $\mathrm{C}(31)$ & $0.9093(7)$ & $0.3989(4)$ & $0.9078(6)$ & $2.5(2)$ \\
\hline$C(32)$ & $0.8670(7)$ & $0.4504(4)$ & $0.8427(7)$ & $2.8(2)$ \\
\hline C(33) & $0.8142(7)$ & $0.4357(4)$ & $0.7411(6)$ & $2.1(2)$ \\
\hline C(34) & $0.8068(6)$ & $0.3705(4)$ & $0.7079(5)$ & $2.2(2)$ \\
\hline$C(35)$ & $1.213(2)$ & $0.5176(10)$ & $0.665(1)$ & $3.9(4)$ \\
\hline$C(36)$ & $1.1569(9)$ & $0.4975(6)$ & $0.5669(8)$ & $4.9(2)$ \\
\hline $\mathrm{C}(37)$ & $1.0300(8)$ & $0.5094(5)$ & $0.5531(7)$ & $3.8(2)$ \\
\hline$C(100)$ & 1.0974 & 0.2729 & 0.5818 & 0.2000 \\
\hline $\mathrm{C}(101)$ & 0.9338 & 0.1085 & 0.4719 & 0.2000 \\
\hline $\mathrm{H}(1)$ & 1.0596 & 0.2863 & 0.7352 & 4.3625 \\
\hline $\mathrm{H}(2)$ & 0.9851 & 0.3572 & 0.5758 & 4.7086 \\
\hline $\mathrm{H}(3)$ & 1.0730 & 0.3138 & 0.4265 & 5.5321 \\
\hline $\mathrm{H}(4)$ & 1.1915 & 0.2131 & 0.4908 & 2.9654 \\
\hline $\mathrm{H}(5)$ & 1.1779 & 0.1951 & 0.6840 & 3.5953 \\
\hline $\mathrm{H}(6)$ & 0.6800 & 0.1466 & 0.4555 & 3.9323 \\
\hline $\mathrm{H}(7)$ & 0.6820 & 0.0739 & 0.4199 & 3.9323 \\
\hline $\mathrm{H}(8)$ & 0.7004 & 0.0906 & 0.5367 & 3.9323 \\
\hline $\mathrm{H}(9)$ & 0.8505 & 0.1907 & 0.2659 & 3.7528 \\
\hline $\mathrm{H}(10)$ & 0.8398 & 0.1172 & 0.2345 & 3.7528 \\
\hline $\mathrm{H}(11)$ & 0.7485 & 0.1505 & 0.2837 & 3.7528 \\
\hline $\mathrm{H}(12)$ & 1.1572 & 0.1239 & 0.3839 & 2.5634 \\
\hline $\mathrm{H}(13)$ & 1.0682 & 0.1020 & 0.2909 & 2.5634 \\
\hline $\mathrm{H}(14)$ & 1.0790 & 0.1756 & 0.3221 & 2.5634 \\
\hline
\end{tabular}




$\begin{array}{lllll}\mathrm{H}(15) & 1.1242 & 0.0538 & 0.6448 & 3.2070 \\ \mathrm{H}(16) & 1.1562 & 0.0334 & 0.5408 & 3.2070 \\ \mathrm{H}(17) & 1.1861 & 0.1029 & 0.5857 & 3.2070 \\ \mathrm{H}(18) & 0.8280 & 0.0532 & 0.6550 & 3.0188 \\ \mathrm{H}(19) & 0.9242 & 0.0060 & 0.6441 & 3.0188 \\ \mathrm{H}(20) & 0.9479 & 0.0699 & 0.7086 & 3.0188 \\ \mathrm{H}(21) & 1.0761 & 0.1594 & 0.8240 & 3.2606 \\ \mathrm{H}(22) & 1.1538 & 0.0956 & 0.9673 & 3.9168 \\ \mathrm{H}(23) & 1.0510 & 0.0638 & 1.0875 & 3.6505 \\ \mathrm{H}(24) & 0.8656 & 0.0946 & 1.0658 & 3.3445 \\ \mathrm{H}(25) & 0.7846 & 0.1587 & 0.9242 & 2.9734 \\ \mathrm{H}(26) & 0.6880 & 0.1001 & 0.7672 & 2.4695 \\ \mathrm{H}(27) & 0.5012 & 0.0743 & 0.7451 & 3.0514 \\ \mathrm{H}(28) & 0.3674 & 0.1490 & 0.6660 & 3.5336 \\ \mathrm{H}(29) & 0.4223 & 0.2512 & 0.6111 & 3.3103 \\ \mathrm{H}(30) & 0.6090 & 0.2763 & 0.6299 & 2.8060 \\ \mathrm{H}(31) & 0.9280 & 0.2999 & 0.9199 & 2.6975 \\ \mathrm{H}(32) & 0.9459 & 0.4083 & 0.9763 & 3.0090 \\ \mathrm{H}(33) & 0.8734 & 0.4949 & 0.8662 & 3.3660 \\ \mathrm{H}(34) & 0.7840 & 0.4699 & 0.6956 & 2.4953 \\ \mathrm{H}(35) & 0.7725 & 0.3612 & 0.6385 & 2.6386 \\ \mathrm{H}(36) & 0.8451 & 0.2794 & 0.3734 & 3.5894 \\ \mathrm{H}(37) & 0.8135 & 0.3092 & 0.4724 & 3.5894 \\ \mathrm{H}(38) & 0.7557 & 0.2457 & 0.4246 & 3.5894 \\ & & & & \end{array}$

$B_{\text {eq }}=8 / 3 \pi^{2}\left(U_{11}\left(a a^{*}\right)^{2}+U_{22}\left(b b^{*}\right)^{2}+U_{33}\left(c c^{*}\right)^{2}+2 U_{12}\left(a a^{*} b b^{*}\right) \cos \gamma+2 U_{13}\left(a a^{*} c c^{*}\right) \cos \beta+\right.$ $\left.2 \mathrm{U}_{23}\left(\mathrm{bb}^{*} \mathrm{cc} *\right) \cos \alpha\right)$

Table 26. Anisotropic Displacement Parameters for $\mathrm{CpCp} * \mathrm{Hf}\left(\mathrm{SnPh}_{3}\right) \mathrm{Me} \bullet 0.5\left(\mathrm{C}_{5} \mathrm{H}_{12}\right)(\mathbf{9})$

$\begin{array}{llllllc}\text { atom } & \mathrm{U}_{11} & \mathrm{U}_{22} & \mathrm{U}_{33} & \mathrm{U}_{12} & \mathrm{U}_{13} & \mathrm{U}_{23} \\ \mathrm{Hf}(1) & 0.0197(2) & 0.0175(2) & 0.0243(2) & 0.0008(1) & 0.0067(1) & 0.0016(1) \\ \mathrm{Sn}(1) & 0.0240(3) & 0.0184(2) & 0.0219(3) & 0.0011(2) & 0.0053(2) & 0.0003(2) \\ \mathrm{C}(1) & 0.027(4) & 0.051(6) & 0.069(6) & -0.023(5) & 0.009(4) & -0.029(5) \\ \mathrm{C}(2) & 0.043(5) & 0.019(4) & 0.103(8) & -0.014(4) & 0.030(6) & -0.010(5) \\ \mathrm{C}(3) & 0.050(5) & 0.043(6) & 0.078(7) & -0.008(5) & 0.017(5) & 0.021(5) \\ \mathrm{C}(4) & 0.024(4) & 0.028(4) & 0.053(5) & -0.008(4) & 0.021(4) & -0.005(4) \\ \mathrm{C}(5) & 0.019(4) & 0.039(5) & 0.049(5) & -0.006(4) & 0.009(4) & -0.016(4) \\ \mathrm{C}(6) & 0.025(4) & 0.023(4) & 0.032(4) & -0.006(3) & 0.002(3) & -0.006(3) \\ \mathrm{C}(7) & 0.027(4) & 0.026(4) & 0.021(4) & 0.001(3) & -0.004(3) & -0.008(3) \\ \mathrm{C}(8) & 0.031(4) & 0.021(4) & 0.021(3) & -0.003(3) & 0.002(3) & 0.004(3) \\ \mathrm{C}(9) & 0.017(3) & 0.024(4) & 0.025(4) & 0.002(3) & 0.001(3) & 0.000(3) \\ \mathrm{C}(10) & 0.030(4) & 0.019(3) & 0.019(4) & -0.002(3) & 0.003(3) & -0.002(3) \\ \mathrm{C}(11) & 0.028(4) & 0.051(6) & 0.046(5) & 0.003(4) & 0.003(4) & -0.012(4) \\ \mathrm{C}(12) & 0.039(5) & 0.053(6) & 0.024(4) & 0.010(4) & -0.008(4) & -0.004(4) \\ \mathrm{C}(13) & 0.037(4) & 0.031(4) & 0.028(4) & 0.005(4) & 0.020(4) & 0.003(3)\end{array}$




$\begin{array}{lrrrrrr}\mathrm{C}(14) & 0.032(4) & 0.021(4) & 0.039(4) & 0.005(3) & -0.003(4) & -0.005(3) \\ \mathrm{C}(15) & 0.048(5) & 0.019(4) & 0.037(4) & -0.002(4) & 0.018(4) & -0.005(3) \\ \mathrm{C}(16) & 0.049(5) & 0.035(4) & 0.032(4) & 0.008(4) & -0.001(4) & 0.006(4) \\ \mathrm{C}(17) & 0.029(4) & 0.022(4) & 0.025(4) & -0.001(3) & 0.004(3) & -0.009(3) \\ \mathrm{C}(18) & 0.031(4) & 0.039(5) & 0.027(4) & -0.003(4) & 0.005(3) & -0.007(4) \\ \mathrm{C}(19) & 0.041(5) & 0.041(5) & 0.036(5) & 0.011(4) & -0.012(4) & -0.009(4) \\ \mathrm{C}(20) & 0.053(6) & 0.040(5) & 0.022(4) & 0.002(4) & -0.006(4) & 0.004(4) \\ \mathrm{C}(21) & 0.050(5) & 0.041(5) & 0.022(4) & -0.016(4) & 0.004(4) & 0.005(4) \\ \mathrm{C}(22) & 0.030(4) & 0.034(4) & 0.027(4) & 0.001(4) & 0.008(3) & -0.005(3) \\ \mathrm{C}(23) & 0.033(4) & 0.019(4) & 0.020(3) & 0.009(3) & 0.011(3) & -0.001(3) \\ \mathrm{C}(24) & 0.026(4) & 0.035(4) & 0.026(4) & -0.005(4) & 0.012(3) & -0.003(3) \\ \mathrm{C}(25) & 0.038(5) & 0.034(4) & 0.036(4) & -0.007(4) & 0.016(4) & -0.007(4) \\ \mathrm{C}(26) & 0.020(4) & 0.054(5) & 0.029(4) & -0.001(4) & -0.005(3) & -0.008(4) \\ \mathrm{C}(27) & 0.035(4) & 0.039(5) & 0.028(4) & 0.016(4) & -0.007(4) & -0.007(4) \\ \mathrm{C}(28) & 0.031(4) & 0.032(4) & 0.025(4) & 0.007(4) & -0.007(3) & -0.002(3) \\ \mathrm{C}(29) & 0.029(4) & 0.025(4) & 0.019(4) & 0.005(3) & 0.009(3) & -0.006(3) \\ \mathrm{C}(30) & 0.027(4) & 0.030(4) & 0.029(4) & -0.001(4) & 0.002(3) & 0.000(3) \\ \mathrm{C}(31) & 0.033(4) & 0.028(4) & 0.035(4) & 0.002(4) & -0.003(4) & -0.006(4) \\ \mathrm{C}(32) & 0.051(5) & 0.020(4) & 0.052(5) & -0.002(4) & 0.025(5) & -0.008(4) \\ \mathrm{C}(33) & 0.036(4) & 0.028(4) & 0.028(4) & 0.009(4) & 0.010(4) & 0.006(3) \\ \mathrm{C}(34) & 0.035(4) & 0.019(4) & 0.034(4) & -0.008(3) & 0.010(4) & -0.001(3)\end{array}$

The general temperature factor expression:

$\exp \left(-2 \pi^{2}\left(a^{*} U_{11} h^{2}+b * 2 U_{22} k^{2}+c^{* 2} U_{33} l^{2}+2 a * b * U_{12} h k+2 a * c * U_{13} h l+2 b * c * U_{23} k 1\right)\right)$

Table 27. Bond Lengths $(\AA)$ for $\mathrm{CpCp} * \mathrm{Hf}\left(\mathrm{SnPh}_{3}\right) \mathrm{Me} \cdot 0.5\left(\mathrm{C}_{5} \mathrm{H}_{12}\right)(\mathbf{9})$

$\begin{array}{llllll}\text { atom } & \text { atom } & \text { distance } & \text { atom } & \text { atom } & \text { distance } \\ \mathrm{Hf}(1) & \mathrm{Sn}(1) & 2.9740(5) & \mathrm{Hf}(1) & \mathrm{C}(1) & 2.516(8) \\ \mathrm{Hf}(1) & \mathrm{C}(2) & 2.508(8) & \mathrm{Hf}(1) & \mathrm{C}(3) & 2.498(9) \\ \mathrm{Hf}(1) & \mathrm{C}(4) & 2.482(7) & \mathrm{Hf}(1) & \mathrm{C}(5) & 2.490(7) \\ \mathrm{Hf}(1) & \mathrm{C}(6) & 2.526(7) & \mathrm{Hf}(1) & \mathrm{C}(7) & 2.512(7) \\ \mathrm{Hf}(1) & \mathrm{C}(8) & 2.473(7) & \mathrm{Hf}(1) & \mathrm{C}(9) & 2.478(7) \\ \mathrm{Hf}(1) & \mathrm{C}(10) & 2.520(7) & \mathrm{Hf}(1) & \mathrm{C}(16) & 2.240(8) \\ \mathrm{Hf}(1) & \mathrm{C}(100) & 2.1893(3) & \mathrm{Hf}(1) & \mathrm{C}(101) & 2.1920(3) \\ \mathrm{Sn}(1) & \mathrm{C}(17) & 2.180(7) & \mathrm{Sn}(1) & \mathrm{C}(23) & 2.168(7) \\ \mathrm{Sn}(1) & \mathrm{C}(29) & 2.189(7) & \mathrm{C}(1) & \mathrm{C}(2) & 1.42(1) \\ \mathrm{C}(1) & \mathrm{C}(5) & 1.40(1) & \mathrm{C}(1) & \mathrm{C}(100) & 1.197(10) \\ \mathrm{C}(2) & \mathrm{C}(3) & 1.43(1) & \mathrm{C}(2) & \mathrm{C}(100) & 1.206(9) \\ \mathrm{C}(3) & \mathrm{C}(4) & 1.42(1) & \mathrm{C}(3) & \mathrm{C}(100) & 1.213(10) \\ \mathrm{C}(4) & \mathrm{C}(5) & 1.42(1) & \mathrm{C}(4) & \mathrm{C}(100) & 1.203(8) \\ \mathrm{C}(5) & \mathrm{C}(100) & 1.208(9) & \mathrm{C}(6) & \mathrm{C}(7) & 1.426(10) \\ \mathrm{C}(6) & \mathrm{C}(10) & 1.427(10) & \mathrm{C}(6) & \mathrm{C}(11) & 1.50(1) \\ \mathrm{C}(6) & \mathrm{C}(101) & 1.213(7) & \mathrm{C}(7) & \mathrm{C}(8) & 1.41(1) \\ \mathrm{C}(7) & \mathrm{C}(12) & 1.523(10) & \mathrm{C}(7) & \mathrm{C}(101) & 1.199(7) \\ \mathrm{C}(8) & \mathrm{C}(9) & 1.424(10) & \mathrm{C}(8) & \mathrm{C}(13) & 1.522(9)\end{array}$




$\begin{array}{llllll}\mathrm{C}(8) & \mathrm{C}(101) & 1.215(7) & \mathrm{C}(9) & \mathrm{C}(10) & 1.41(1) \\ \mathrm{C}(9) & \mathrm{C}(14) & 1.518(10) & \mathrm{C}(9) & \mathrm{C}(101) & 1.197(7) \\ \mathrm{C}(10) & \mathrm{C}(15) & 1.502(10) & \mathrm{C}(10) & \mathrm{C}(101) & 1.209(7) \\ \mathrm{C}(17) & \mathrm{C}(18) & 1.39(1) & \mathrm{C}(17) & \mathrm{C}(22) & 1.404(10) \\ \mathrm{C}(18) & \mathrm{C}(19) & 1.41(1) & \mathrm{C}(19) & \mathrm{C}(20) & 1.35(1) \\ \mathrm{C}(20) & \mathrm{C}(21) & 1.38(1) & \mathrm{C}(21) & \mathrm{C}(22) & 1.40(1) \\ \mathrm{C}(23) & \mathrm{C}(24) & 1.40(1) & \mathrm{C}(23) & \mathrm{C}(28) & 1.41(1) \\ \mathrm{C}(24) & \mathrm{C}(25) & 1.39(1) & \mathrm{C}(25) & \mathrm{C}(26) & 1.39(1) \\ \mathrm{C}(26) & \mathrm{C}(27) & 1.38(1) & \mathrm{C}(27) & \mathrm{C}(28) & 1.39(1) \\ \mathrm{C}(29) & \mathrm{C}(30) & 1.41(1) & \mathrm{C}(29) & \mathrm{C}(34) & 1.38(1) \\ \mathrm{C}(30) & \mathrm{C}(31) & 1.38(1) & \mathrm{C}(31) & \mathrm{C}(32) & 1.39(1) \\ \mathrm{C}(32) & \mathrm{C}(33) & 1.41(1) & \mathrm{C}(33) & \mathrm{C}(34) & 1.39(1) \\ \mathrm{C}(35) & \mathrm{C}(36) & 1.41(2) & \mathrm{C}(36) & \mathrm{C}(37) & 1.56(1) \\ \mathrm{C}(37) & \mathrm{C}(37) & 1.51(2) & & & \end{array}$

Table 28. Bond Angles $\left({ }^{\circ}\right)$ for $\mathrm{CpCp} * \mathrm{Hf}\left(\mathrm{SnPh}_{3}\right) \mathrm{Me} \bullet 0.5\left(\mathrm{C}_{5} \mathrm{H}_{12}\right)(\mathbf{9})$

\begin{tabular}{|c|c|c|c|c|c|c|c|}
\hline atom & atom & atom & angle & atom & atom & atom & angle \\
\hline $\operatorname{Sn}(1)$ & $\mathrm{Hf}(1)$ & $\mathrm{C}(1)$ & $73.1(2)$ & $\operatorname{Sn}(1)$ & $\operatorname{Hf}(1)$ & $\mathrm{C}(2)$ & $89.0(2)$ \\
\hline $\operatorname{Sn}(1)$ & $\mathrm{Hf}(1)$ & $\mathrm{C}(3)$ & $121.8(3)$ & $\operatorname{Sn}(1)$ & $\operatorname{Hf}(1)$ & $\mathrm{C}(4)$ & $125.6(2)$ \\
\hline $\operatorname{Sn}(1)$ & $\mathrm{Hf}(1)$ & $\mathrm{C}(5)$ & $93.9(2)$ & $\operatorname{Sn}(1)$ & $\operatorname{Hf}(1)$ & $C(6)$ & $89.0(2)$ \\
\hline $\operatorname{Sn}(1)$ & $\mathrm{Hf}(1)$ & $C(7)$ & $121.0(2)$ & $\operatorname{Sn}(1)$ & $\operatorname{Hf}(1)$ & $\mathrm{C}(8)$ & $137.5(2)$ \\
\hline $\operatorname{Sn}(1)$ & $\operatorname{Hf}(1)$ & $\mathrm{C}(9)$ & 109.3(2) & $\operatorname{Sn}(1)$ & $\operatorname{Hf}(1)$ & $\mathrm{C}(10)$ & $82.6(2)$ \\
\hline $\operatorname{Sn}(1)$ & $\mathrm{Hf}(1)$ & $C(16)$ & $92.7(2)$ & $\operatorname{Sn}(1)$ & $\operatorname{Hf}(1)$ & $C(100)$ & $101.35(1)$ \\
\hline $\operatorname{Sn}(1)$ & $\mathrm{Hf}(1)$ & $C(101)$ & $108.98(1)$ & $\mathrm{C}(1)$ & $\operatorname{Hf}(1)$ & $C(2)$ & $32.8(3)$ \\
\hline$C(1)$ & $\mathrm{Hf}(1)$ & $\mathrm{C}(3)$ & $54.5(3)$ & $\mathrm{C}(1)$ & $\operatorname{Hf}(1)$ & $C(4)$ & $54.3(3)$ \\
\hline$C(1)$ & $\mathrm{Hf}(1)$ & $\mathrm{C}(5)$ & $32.5(3)$ & $\mathrm{C}(1)$ & $\operatorname{Hf}(1)$ & $C(6)$ & $155.3(3)$ \\
\hline$C(1)$ & $\mathrm{Hf}(1)$ & $C(7)$ & $163.4(3)$ & $\mathrm{C}(1)$ & $\operatorname{Hf}(1)$ & $\mathrm{C}(8)$ & 131.1(3) \\
\hline$C(1)$ & $\mathrm{Hf}(1)$ & $\mathrm{C}(9)$ & $115.0(3)$ & $\mathrm{C}(1)$ & $\operatorname{Hf}(1)$ & $C(10)$ & $125.3(3)$ \\
\hline$C(1)$ & $\mathrm{Hf}(1)$ & $C(16)$ & $109.6(3)$ & $\mathrm{C}(1)$ & $\operatorname{Hf}(1)$ & $C(100)$ & $28.4(2)$ \\
\hline$C(1)$ & $\mathrm{Hf}(1)$ & $C(101)$ & $143.7(2)$ & $\mathrm{C}(2)$ & $\operatorname{Hf}(1)$ & $C(3)$ & $33.1(3)$ \\
\hline$C(2)$ & $\mathrm{Hf}(1)$ & $C(4)$ & $54.7(3)$ & $C(2)$ & $\operatorname{Hf}(1)$ & $C(5)$ & $54.6(3)$ \\
\hline$C(2)$ & $\mathrm{Hf}(1)$ & $C(6)$ & $167.9(3)$ & $\mathrm{C}(2)$ & $\operatorname{Hf}(1)$ & $C(7)$ & $144.7(3)$ \\
\hline$C(2)$ & $\mathrm{Hf}(1)$ & $\mathrm{C}(8)$ & $131.0(3)$ & $C(2)$ & $\operatorname{Hf}(1)$ & $\mathrm{C}(9)$ & 137.1(3) \\
\hline$C(2)$ & $\mathrm{Hf}(1)$ & $C(10)$ & $157.9(3)$ & $\mathrm{C}(2)$ & $\operatorname{Hf}(1)$ & $\mathrm{C}(16)$ & $80.7(3)$ \\
\hline$C(2)$ & $\mathrm{Hf}(1)$ & $C(100)$ & $28.7(2)$ & $C(2)$ & $\operatorname{Hf}(1)$ & $C(101)$ & $160.0(2)$ \\
\hline$C(3)$ & $\mathrm{Hf}(1)$ & $C(4)$ & $33.1(3)$ & $C(3)$ & $\operatorname{Hf}(1)$ & $C(5)$ & $55.0(3)$ \\
\hline$C(3)$ & $\mathrm{Hf}(1)$ & $C(6)$ & $148.2(3)$ & $\mathrm{C}(3)$ & $\operatorname{Hf}(1)$ & $C(7)$ & $115.4(3)$ \\
\hline$C(3)$ & $\mathrm{Hf}(1)$ & $\mathrm{C}(8)$ & $98.3(3)$ & $\mathrm{C}(3)$ & $\operatorname{Hf}(1)$ & $C(9)$ & 114.1(3) \\
\hline$C(3)$ & $\mathrm{Hf}(1)$ & $C(10)$ & $146.7(3)$ & $\mathrm{C}(3)$ & $\operatorname{Hf}(1)$ & $\mathrm{C}(16)$ & $83.8(3)$ \\
\hline$C(3)$ & $\mathrm{Hf}(1)$ & $C(100)$ & $29.0(2)$ & $C(3)$ & $\operatorname{Hf}(1)$ & $C(101)$ & $127.7(3)$ \\
\hline C(4) & $\mathrm{Hf}(1)$ & $\mathrm{C}(5)$ & $33.2(3)$ & $\mathrm{C}(4)$ & $\operatorname{Hf}(1)$ & $C(6)$ & $134.6(2)$ \\
\hline C(4) & $\operatorname{Hf}(1)$ & $\mathrm{C}(7)$ & $109.7(3)$ & $\mathrm{C}(4)$ & $\operatorname{Hf}(1)$ & $\mathrm{C}(8)$ & $80.5(2)$ \\
\hline C(4) & $\mathrm{Hf}(1)$ & $\mathrm{C}(9)$ & $84.1(3)$ & $\mathrm{C}(4)$ & $\operatorname{Hf}(1)$ & $\mathrm{C}(10)$ & $115.3(3)$ \\
\hline $\mathrm{C}(4)$ & $\mathrm{Hf}(1)$ & $C(16)$ & $115.5(3)$ & $\mathrm{C}(4)$ & $\operatorname{Hf}(1)$ & $C(100)$ & $29.0(2)$ \\
\hline C(4) & $\mathrm{Hf}(1)$ & $C(101)$ & 106.1(2) & $C(5)$ & $\operatorname{Hf}(1)$ & $\mathrm{C}(6)$ & 137.4(3) \\
\hline
\end{tabular}




\begin{tabular}{|c|c|c|c|c|c|c|c|}
\hline$C(5)$ & $\mathrm{Hf}(1)$ & $C(7)$ & $131.9(2)$ & $C(5)$ & $\mathrm{Hf}(1)$ & $C(8)$ & $99.1(2)$ \\
\hline$C(5)$ & $\mathrm{Hf}(1)$ & $\mathrm{C}(9)$ & $84.9(3)$ & $C(5)$ & $\mathrm{Hf}(1)$ & $\mathrm{C}(10)$ & $105.5(3)$ \\
\hline$C(5)$ & $\mathrm{Hf}(1)$ & $C(16)$ & $134.6(3)$ & $C(5)$ & $\mathrm{Hf}(1)$ & $C(100)$ & $29.0(2)$ \\
\hline$C(5)$ & $\mathrm{Hf}(1)$ & $\mathrm{C}(101)$ & $113.6(2)$ & $C(6)$ & $\mathrm{Hf}(1)$ & $\mathrm{C}(7)$ & $32.9(2)$ \\
\hline$C(6)$ & $\mathrm{Hf}(1)$ & $\mathrm{C}(8)$ & $55.0(2)$ & $C(6)$ & $\operatorname{Hf}(1)$ & $\mathrm{C}(9)$ & $54.5(2)$ \\
\hline$C(6)$ & $\mathrm{Hf}(1)$ & $\mathrm{C}(10)$ & $32.9(2)$ & $\mathrm{C}(6)$ & $\mathrm{Hf}(1)$ & $C(16)$ & $87.6(3)$ \\
\hline$C(6)$ & $\mathrm{Hf}(1)$ & $\mathrm{C}(100)$ & $162.5(2)$ & $C(6)$ & $\operatorname{Hf}(1)$ & $C(101)$ & $28.7(2)$ \\
\hline$C(7)$ & $\mathrm{Hf}(1)$ & $\mathrm{C}(8)$ & $32.8(2)$ & $\mathrm{C}(7)$ & $\operatorname{Hf}(1)$ & $\mathrm{C}(9)$ & $54.3(2)$ \\
\hline$C(7)$ & $\mathrm{Hf}(1)$ & $\mathrm{C}(10)$ & $54.3(2)$ & $C(7)$ & $\operatorname{Hf}(1)$ & $C(16)$ & $79.9(3)$ \\
\hline$C(7)$ & $\mathrm{Hf}(1)$ & $\mathrm{C}(100)$ & $137.2(2)$ & $\mathrm{C}(7)$ & $\mathrm{Hf}(1)$ & $\mathrm{C}(101)$ & $28.5(2)$ \\
\hline $\mathrm{C}(8)$ & $\mathrm{Hf}(1)$ & $\mathrm{C}(9)$ & $33.4(2)$ & $\mathrm{C}(8)$ & $\mathrm{Hf}(1)$ & $C(10)$ & $55.0(2)$ \\
\hline$C(8)$ & $\mathrm{Hf}(1)$ & $C(16)$ & $105.8(3)$ & $\mathrm{C}(8)$ & $\mathrm{Hf}(1)$ & $\mathrm{C}(100)$ & $109.4(2)$ \\
\hline $\mathrm{C}(8)$ & $\mathrm{Hf}(1)$ & $\mathrm{C}(101)$ & $29.4(2)$ & $\mathrm{C}(9)$ & $\mathrm{Hf}(1)$ & $\mathrm{C}(10)$ & $32.7(2)$ \\
\hline$C(9)$ & $\mathrm{Hf}(1)$ & $\mathrm{C}(16)$ & $134.2(3)$ & $\mathrm{C}(9)$ & $\mathrm{Hf}(1)$ & $\mathrm{C}(100)$ & $108.4(2)$ \\
\hline $\mathrm{C}(9)$ & $\mathrm{Hf}(1)$ & $\mathrm{C}(101)$ & $28.9(2)$ & $C(10)$ & $\operatorname{Hf}(1)$ & $C(16)$ & $119.9(3)$ \\
\hline$C(10)$ & $\mathrm{Hf}(1)$ & $\mathrm{C}(100)$ & $134.0(2)$ & $C(10)$ & $\operatorname{Hf}(1)$ & $\mathrm{C}(101)$ & $28.7(1)$ \\
\hline$C(16)$ & $\mathrm{Hf}(1)$ & $\mathrm{C}(100)$ & $105.8(2)$ & $C(16)$ & $\operatorname{Hf}(1)$ & $\mathrm{C}(101)$ & $106.5(2)$ \\
\hline$C(100)$ & $\mathrm{Hf}(1)$ & $\mathrm{C}(101)$ & $133.96(1)$ & $\operatorname{Hf}(1)$ & $\operatorname{Sn}(1)$ & $\mathrm{C}(17)$ & $117.0(2)$ \\
\hline $\mathrm{Hf}(1)$ & $\operatorname{Sn}(1)$ & $\mathrm{C}(23)$ & $120.1(2)$ & $\operatorname{Hf}(1)$ & $\operatorname{Sn}(1)$ & $\mathrm{C}(29)$ & $110.2(2)$ \\
\hline$C(17)$ & $\operatorname{Sn}(1)$ & $\mathrm{C}(23)$ & $102.7(3)$ & $\mathrm{C}(17)$ & $\operatorname{Sn}(1)$ & $\mathrm{C}(29)$ & $101.9(3)$ \\
\hline $\mathrm{C}(23)$ & $\operatorname{Sn}(1)$ & $\mathrm{C}(29)$ & $102.5(3)$ & $\operatorname{Hf}(1)$ & $\mathrm{C}(1)$ & $\mathrm{C}(2)$ & $73.3(5)$ \\
\hline $\mathrm{Hf}(1)$ & $\mathrm{C}(1)$ & $\mathrm{C}(5)$ & $72.7(5)$ & $\operatorname{Hf}(1)$ & $\mathrm{C}(1)$ & $\mathrm{C}(100)$ & $60.5(3)$ \\
\hline $\mathrm{C}(2)$ & $\mathrm{C}(1)$ & $C(5)$ & $108.9(8)$ & $\mathrm{C}(2)$ & $\mathrm{C}(1)$ & $C(100)$ & $54.2(6)$ \\
\hline$C(5)$ & $\mathrm{C}(1)$ & $\mathrm{C}(100)$ & $54.7(5)$ & $\operatorname{Hf}(1)$ & $\mathrm{C}(2)$ & $\mathrm{C}(1)$ & $73.9(5)$ \\
\hline $\mathrm{Hf}(1)$ & $\mathrm{C}(2)$ & $\mathrm{C}(3)$ & $73.1(5)$ & $\operatorname{Hf}(1)$ & $\mathrm{C}(2)$ & $\mathrm{C}(100)$ & $60.8(3)$ \\
\hline$C(1)$ & $\mathrm{C}(2)$ & $\mathrm{C}(3)$ & $107.7(8)$ & $\mathrm{C}(1)$ & $\mathrm{C}(2)$ & $C(100)$ & $53.6(6)$ \\
\hline$C(3)$ & $\mathrm{C}(2)$ & $\mathrm{C}(100)$ & $54.1(5)$ & $\operatorname{Hf}(1)$ & $\mathrm{C}(3)$ & $\mathrm{C}(2)$ & $73.9(5)$ \\
\hline $\mathrm{Hf}(1)$ & $\mathrm{C}(3)$ & $\mathrm{C}(4)$ & $72.8(5)$ & $\operatorname{Hf}(1)$ & $\mathrm{C}(3)$ & $C(100)$ & $61.2(4)$ \\
\hline$C(2)$ & $\mathrm{C}(3)$ & $\mathrm{C}(4)$ & $107.4(8)$ & $\mathrm{C}(2)$ & $\mathrm{C}(3)$ & $C(100)$ & $53.7(6)$ \\
\hline$C(4)$ & $\mathrm{C}(3)$ & $\mathrm{C}(100)$ & $53.7(5)$ & $\operatorname{Hf}(1)$ & $\mathrm{C}(4)$ & $\mathrm{C}(3)$ & $74.0(5)$ \\
\hline $\mathrm{Hf}(1)$ & $\mathrm{C}(4)$ & $\mathrm{C}(5)$ & $73.7(4)$ & $\operatorname{Hf}(1)$ & $\mathrm{C}(4)$ & $\mathrm{C}(100)$ & $61.9(3)$ \\
\hline$C(3)$ & $\mathrm{C}(4)$ & $C(5)$ & $108.3(8)$ & $\mathrm{C}(3)$ & $\mathrm{C}(4)$ & $C(100)$ & $54.3(5)$ \\
\hline$C(5)$ & $\mathrm{C}(4)$ & $\mathrm{C}(100)$ & $54.0(5)$ & $\operatorname{Hf}(1)$ & $\mathrm{C}(5)$ & $\mathrm{C}(1)$ & $74.8(5)$ \\
\hline $\mathrm{Hf}(1)$ & $\mathrm{C}(5)$ & $\mathrm{C}(4)$ & $73.1(4)$ & $\operatorname{Hf}(1)$ & $\mathrm{C}(5)$ & $\mathrm{C}(100)$ & $61.6(3)$ \\
\hline$C(1)$ & $\mathrm{C}(5)$ & $\mathrm{C}(4)$ & $107.7(8)$ & $\mathrm{C}(1)$ & $\mathrm{C}(5)$ & $\mathrm{C}(100)$ & $54.0(6)$ \\
\hline$C(4)$ & $C(5)$ & $\mathrm{C}(100)$ & $53.7(5)$ & $\operatorname{Hf}(1)$ & $\mathrm{C}(6)$ & $\mathrm{C}(7)$ & $73.0(4)$ \\
\hline $\mathrm{Hf}(1)$ & $\mathrm{C}(6)$ & $\mathrm{C}(10)$ & $73.3(4)$ & $\operatorname{Hf}(1)$ & $\mathrm{C}(6)$ & $\mathrm{C}(11)$ & $125.4(5)$ \\
\hline $\mathrm{Hf}(1)$ & $\mathrm{C}(6)$ & $\mathrm{C}(101)$ & $60.2(3)$ & $C(7)$ & $\mathrm{C}(6)$ & $\mathrm{C}(10)$ & $107.1(6)$ \\
\hline$C(7)$ & $C(6)$ & $\mathrm{C}(11)$ & $125.8(7)$ & $C(7)$ & $\mathrm{C}(6)$ & $\mathrm{C}(101)$ & $53.3(4)$ \\
\hline$C(10)$ & $C(6)$ & $\mathrm{C}(11)$ & $126.6(7)$ & $\mathrm{C}(10)$ & $\mathrm{C}(6)$ & $\mathrm{C}(101)$ & $53.8(4)$ \\
\hline $\mathrm{C}(11)$ & $C(6)$ & $\mathrm{C}(101)$ & $174.4(7)$ & $\operatorname{Hf}(1)$ & $\mathrm{C}(7)$ & $\mathrm{C}(6)$ & $74.1(4)$ \\
\hline $\mathrm{Hf}(1)$ & $\mathrm{C}(7)$ & $\mathrm{C}(8)$ & $72.1(4)$ & $\operatorname{Hf}(1)$ & $\mathrm{C}(7)$ & $\mathrm{C}(12)$ & $125.0(5)$ \\
\hline $\mathrm{Hf}(1)$ & $\mathrm{C}(7)$ & $\mathrm{C}(101)$ & $60.7(3)$ & $\mathrm{C}(6)$ & $\mathrm{C}(7)$ & $\mathrm{C}(8)$ & $109.0(6)$ \\
\hline$C(6)$ & $\mathrm{C}(7)$ & $\mathrm{C}(12)$ & $125.1(7)$ & $\mathrm{C}(6)$ & $\mathrm{C}(7)$ & $\mathrm{C}(101)$ & $54.2(4)$ \\
\hline$C(8)$ & $\mathrm{C}(7)$ & $\mathrm{C}(12)$ & $125.6(7)$ & $\mathrm{C}(8)$ & $\mathrm{C}(7)$ & $\mathrm{C}(101)$ & $54.8(4)$ \\
\hline$C(12)$ & $\mathrm{C}(7)$ & $\mathrm{C}(101)$ & $174.2(7)$ & $\operatorname{Hf}(1)$ & $\mathrm{C}(8)$ & $\mathrm{C}(7)$ & $75.1(4)$ \\
\hline
\end{tabular}




\begin{tabular}{|c|c|c|c|c|c|c|c|}
\hline $\mathrm{Hf}(1)$ & $\mathrm{C}(8)$ & $\mathrm{C}(9)$ & $73.5(4)$ & $\operatorname{Hf}(1)$ & $\mathrm{C}(8)$ & $\mathrm{C}(13)$ & $123.3(5)$ \\
\hline $\operatorname{Hf}(1)$ & $\mathrm{C}(8)$ & $C(101)$ & $62.4(3)$ & $C(7)$ & $C(8)$ & $\mathrm{C}(9)$ & $107.0(6)$ \\
\hline $\mathrm{C}(7)$ & $\mathrm{C}(8)$ & $C(13)$ & $124.6(6)$ & $C(7)$ & $C(8)$ & $C(101)$ & $53.8(4)$ \\
\hline$C(9)$ & $\mathrm{C}(8)$ & $C(13)$ & $127.9(7)$ & $C(9)$ & $\mathrm{C}(8)$ & $C(101)$ & $53.2(4)$ \\
\hline$C(13)$ & $C(8)$ & $C(101)$ & 174.2(7) & $\mathrm{Hf}(1)$ & $\mathrm{C}(9)$ & $\mathrm{C}(8)$ & 73.1(4) \\
\hline $\mathrm{Hf}(1)$ & $C(9)$ & $C(10)$ & $75.3(4)$ & $\operatorname{Hf}(1)$ & $\mathrm{C}(9)$ & $C(14)$ & $124.7(5)$ \\
\hline $\mathrm{Hf}(1)$ & $\mathrm{C}(9)$ & $C(101)$ & $62.2(3)$ & $\mathrm{C}(8)$ & $\mathrm{C}(9)$ & $\mathrm{C}(10)$ & $109.0(6)$ \\
\hline $\mathrm{C}(8)$ & $\mathrm{C}(9)$ & $C(14)$ & 126.1(6) & $C(8)$ & $\mathrm{C}(9)$ & $C(101)$ & $54.4(4)$ \\
\hline$C(10)$ & $\mathrm{C}(9)$ & $C(14)$ & $124.3(6)$ & $C(10)$ & $\mathrm{C}(9)$ & $C(101)$ & $54.6(4)$ \\
\hline$C(14)$ & $\mathrm{C}(9)$ & $C(101)$ & 173.1(7) & $\operatorname{Hf}(1)$ & $\mathrm{C}(10)$ & $C(6)$ & $73.8(4)$ \\
\hline $\operatorname{Hf}(1)$ & $\mathrm{C}(10)$ & $\mathrm{C}(9)$ & $72.0(4)$ & $\operatorname{Hf}(1)$ & $C(10)$ & $C(15)$ & $128.3(4)$ \\
\hline $\mathrm{Hf}(1)$ & $\mathrm{C}(10)$ & $C(101)$ & $60.4(3)$ & $C(6)$ & $C(10)$ & $\mathrm{C}(9)$ & $107.8(6)$ \\
\hline $\mathrm{C}(6)$ & $\mathrm{C}(10)$ & $C(15)$ & $125.2(7)$ & $C(6)$ & $C(10)$ & $C(101)$ & $54.0(4)$ \\
\hline C(9) & $\mathrm{C}(10)$ & $C(15)$ & $126.0(6)$ & $C(9)$ & $C(10)$ & $C(101)$ & $53.8(4)$ \\
\hline$C(15)$ & $C(10)$ & $C(101)$ & $171.3(6)$ & $\operatorname{Sn}(1)$ & $C(17)$ & $C(18)$ & $121.5(5)$ \\
\hline $\operatorname{Sn}(1)$ & $\mathrm{C}(17)$ & $C(22)$ & $121.5(5)$ & $C(18)$ & $\mathrm{C}(17)$ & $\mathrm{C}(22)$ & $117.0(7)$ \\
\hline$C(17)$ & $\mathrm{C}(18)$ & $C(19)$ & $120.9(7)$ & $C(18)$ & $\mathrm{C}(19)$ & $\mathrm{C}(20)$ & $121.5(8)$ \\
\hline$C(19)$ & $\mathrm{C}(20)$ & $C(21)$ & $118.8(8)$ & $C(20)$ & $C(21)$ & $C(22)$ & $121.0(7)$ \\
\hline$C(17)$ & $\mathrm{C}(22)$ & $C(21)$ & $120.8(7)$ & $\operatorname{Sn}(1)$ & $C(23)$ & $C(24)$ & $123.4(5)$ \\
\hline $\operatorname{Sn}(1)$ & $\mathrm{C}(23)$ & $C(28)$ & $120.4(5)$ & $C(24)$ & $C(23)$ & $\mathrm{C}(28)$ & $116.2(7)$ \\
\hline$C(23)$ & $C(24)$ & $C(25)$ & $121.6(7)$ & $C(24)$ & $C(25)$ & $C(26)$ & $120.6(8)$ \\
\hline$C(25)$ & $C(26)$ & $C(27)$ & $119.6(7)$ & $C(26)$ & $C(27)$ & $\mathrm{C}(28)$ & $119.6(7)$ \\
\hline$C(23)$ & $\mathrm{C}(28)$ & $C(27)$ & $122.4(8)$ & $\operatorname{Sn}(1)$ & $\mathrm{C}(29)$ & $\mathrm{C}(30)$ & $120.2(5)$ \\
\hline $\operatorname{Sn}(1)$ & $\mathrm{C}(29)$ & $C(34)$ & $121.8(5)$ & $C(30)$ & $\mathrm{C}(29)$ & $\mathrm{C}(34)$ & $117.9(7)$ \\
\hline$C(29)$ & $\mathrm{C}(30)$ & $C(31)$ & $120.9(7)$ & $C(30)$ & $\mathrm{C}(31)$ & $\mathrm{C}(32)$ & $121.0(7)$ \\
\hline $\mathrm{C}(31)$ & $\mathrm{C}(32)$ & $C(33)$ & $118.6(7)$ & $C(32)$ & $\mathrm{C}(33)$ & $C(34)$ & $119.6(7)$ \\
\hline$C(29)$ & $\mathrm{C}(34)$ & $C(33)$ & $122.0(7)$ & $C(35)$ & $C(36)$ & $\mathrm{C}(37)$ & 111(1) \\
\hline$C(36)$ & $\mathrm{C}(37)$ & $C(37)$ & 112(1) & $\operatorname{Hf}(1)$ & $C(100)$ & $\mathrm{C}(1)$ & 91.1(4) \\
\hline $\mathrm{Hf}(1)$ & $C(100)$ & $C(2)$ & $90.5(4)$ & $\mathrm{Hf}(1)$ & $C(100)$ & $\mathrm{C}(3)$ & $89.7(5)$ \\
\hline $\mathrm{Hf}(1)$ & $C(100)$ & $\mathrm{C}(4)$ & $89.1(4)$ & $\operatorname{Hf}(1)$ & $C(100)$ & $\mathrm{C}(5)$ & $89.4(4)$ \\
\hline$C(1)$ & $C(100)$ & $\mathrm{C}(2)$ & 72.2(7) & $\mathrm{C}(1)$ & $C(100)$ & $\mathrm{C}(3)$ & $144.4(7)$ \\
\hline $\mathrm{C}(1)$ & $C(100)$ & $\mathrm{C}(4)$ & $143.5(6)$ & $C(1)$ & $C(100)$ & $\mathrm{C}(5)$ & $71.2(6)$ \\
\hline $\mathrm{C}(2)$ & $C(100)$ & $C(3)$ & $72.2(7)$ & $C(2)$ & $C(100)$ & $\mathrm{C}(4)$ & $144.2(7)$ \\
\hline$C(2)$ & $C(100)$ & $C(5)$ & $143.4(7)$ & $C(3)$ & $C(100)$ & $\mathrm{C}(4)$ & $72.0(6)$ \\
\hline $\mathrm{C}(3)$ & $C(100)$ & $C(5)$ & $144.4(6)$ & $\mathrm{C}(4)$ & $C(100)$ & $\mathrm{C}(5)$ & $72.3(5)$ \\
\hline $\mathrm{Hf}(1)$ & $C(101)$ & $C(6)$ & $91.1(3)$ & $\operatorname{Hf}(1)$ & $C(101)$ & $C(7)$ & $90.8(3)$ \\
\hline $\operatorname{Hf}(1)$ & $C(101)$ & $\mathrm{C}(8)$ & $88.2(3)$ & $\operatorname{Hf}(1)$ & $C(101)$ & $\mathrm{C}(9)$ & $88.9(3)$ \\
\hline $\mathrm{Hf}(1)$ & $C(101)$ & $C(10)$ & $90.9(3)$ & $C(6)$ & $C(101)$ & $C(7)$ & $72.5(5)$ \\
\hline $\mathrm{C}(6)$ & $\mathrm{C}(101)$ & $\mathrm{C}(8)$ & $143.8(5)$ & $C(6)$ & $C(101)$ & $\mathrm{C}(9)$ & $143.8(5)$ \\
\hline$C(6)$ & $C(101)$ & $C(10)$ & $72.2(5)$ & $C(7)$ & $C(101)$ & $\mathrm{C}(8)$ & $71.4(5)$ \\
\hline$C(7)$ & $C(101)$ & $C(9)$ & $143.7(5)$ & $C(7)$ & $C(101)$ & $\mathrm{C}(10)$ & $144.7(5)$ \\
\hline $\mathrm{C}(8)$ & $C(101)$ & $\mathrm{C}(9)$ & $72.4(5)$ & $\mathrm{C}(8)$ & $C(101)$ & $\mathrm{C}(10)$ & $144.0(5)$ \\
\hline$C(9)$ & $C(101)$ & $C(10)$ & $71.6(5)$ & & & & \\
\hline
\end{tabular}


Table 29. Crystal Data for $\mathrm{CpCp} * \mathrm{Hf}\left(\mathrm{SnPh}_{3}\right) \mathrm{OMe} \bullet 0.5\left(\mathrm{C}_{7} \mathrm{H}_{8}\right)(\mathbf{1 0})$

Empirical Formula

Formula Weight

Crystal Color, Habit

Crystal Dimensions

Crystal System

Lattice Type

No. of Reflections Used for Unit

Cell Determination (2 $\theta$ range)

Lattice Parameters
$\mathrm{C}_{37.50} \mathrm{H}_{33} \mathrm{HfSnO}$

796.85

yellow, blocks

$0.20 \times 0.23 \times 0.16 \mathrm{~mm}$

triclinic

Primitive

$5520\left(3.0-51.4^{0}\right)$

$\mathrm{a}=10.6327 \AA$

$\mathrm{b}=11.0716 \AA$

$\mathrm{c}=15.2844 \AA$

$\alpha=82.3920^{\circ}$

$\beta=80.6940^{\circ}$

$\gamma=65.9410^{\circ}$

$\mathrm{V}=1616.9429 \AA^{3}$

$\mathrm{P} \overline{1}(\# 2)$

2

$1.637 \mathrm{~g} / \mathrm{cm}^{3}$

776.00

$40.01 \mathrm{~cm}^{-1}$

Table 30. Intensity Measurements for $\mathrm{CpCp} * \mathrm{Hf}\left(\mathrm{SnPh}_{3}\right) \mathrm{OMe} \bullet 0.5\left(\mathrm{C}_{7} \mathrm{H}_{8}\right)(\mathbf{1 0})$

Diffractometer

Radiation

Crystal to Detector Distance

Temperature

Scan Type

Scan Rate

$2 \theta_{\max }$

No. of Reflections Measured

Corrections
SMART

$\operatorname{MoK} \alpha(1=0.71069 \AA)$

graphite monochromated

$60.0 \mathrm{~mm}$

$-161^{\circ} \mathrm{C}$

$\omega\left(0.3^{\circ}\right.$ per frame $)$

10.0 seconds per frame

$51.4^{\mathrm{O}}$

Total: 8224

Unique: $5198\left(\mathrm{R}_{\text {int }}=0.071\right)$

Lorentz-polarization Absorption

$(\mathrm{Tmax}=0.632, \mathrm{Tmin}=0.423)$ 
Table 31. Structure Solution and Refinement for $\mathrm{CpCp} * \mathrm{Hf}\left(\mathrm{SnPh}_{3}\right) \mathrm{OMe} \bullet 0.5\left(\mathrm{C}_{7} \mathrm{H}_{8}\right)(\mathbf{1 0})$

$\begin{array}{ll}\text { Structure Solution } & \text { Direct Methods (SAPI91) } \\ \text { Refinement } & \text { Full-matrix least-squares } \\ \text { Function Minimized } & \Sigma \omega(|\mathrm{Fol}-| \mathrm{Fcl})^{2} \\ \text { Least Squares Weights } & 1 / \mathrm{\sigma}^{2}(\mathrm{Fo})=4 \mathrm{Fo}^{2} / \mathrm{\sigma}^{2}\left(\mathrm{Fo}^{2}\right) \\ \text { p-factor } & 0.030 \\ \text { Anomalous Dispersion } & \text { All non-hydrogen atoms } \\ \text { No. Observations }(\mathrm{I}>3.00 \sigma(\mathrm{I})) & 4239 \\ \text { No. Variables } & 354 \\ \text { Reflection/Parameter Ratio } & 11.97 \\ \text { Residuals: R; Rw; Rall } & 0.043 ; 0.047 ; 0.051 \\ \text { Goodness of Fit Indicator } & 1.74 \\ \text { Max Shift/Error in Final Cycle } & 0.00 \\ \text { Maximum peak in Final Diff. Map } & 1.80 \mathrm{e}^{-} / \AA^{3} \\ \text { Minimum peak in Final Diff. Map } & -2.98 \mathrm{e}^{-} / \AA^{3}\end{array}$

Table 32. Atomic coordinates and $\mathrm{B}_{\text {iso }} / \mathrm{B}_{\text {eq }}$ for $\mathrm{CpCp} * \mathrm{Hf}\left(\mathrm{SnPh}_{3}\right) \mathrm{OMe} \bullet 0.5\left(\mathrm{C}_{7} \mathrm{H}_{8}\right)(\mathbf{1 0})$

$\begin{array}{lllll}\text { atom } & \mathrm{x} & \mathrm{y} & \mathrm{z} & \mathrm{B}_{\mathrm{eq}} \\ \mathrm{Hf}(1) & 0.07359(3) & 0.25312(3) & 0.80214(2) & 1.681(7) \\ \mathrm{Sn}(1) & -0.18836(5) & 0.29689(5) & 0.73505(3) & 1.65(1) \\ \mathrm{O}(1) & -0.0256(5) & 0.3013(6) & 0.9202(3) & 2.5(1) \\ \mathrm{C}(1) & 0.1168(8) & 0.0271(9) & 0.7529(7) & 3.5(2) \\ \mathrm{C}(2) & 0.0792(8) & 0.0190(8) & 0.8447(7) & 3.2(2) \\ \mathrm{C}(3) & 0.1794(10) & 0.0323(10) & 0.8877(7) & 3.6(2) \\ \mathrm{C}(4) & 0.2807(9) & 0.0489(10) & 0.8206(8) & 3.9(2) \\ \mathrm{C}(5) & 0.2452(10) & 0.0413(10) & 0.7384(8) & 4.3(2) \\ \mathrm{C}(6) & 0.0590(7) & 0.4868(8) & 0.7958(5) & 2.1(2) \\ \mathrm{C}(7) & 0.2001(7) & 0.3961(8) & 0.8050(5) & 1.7(2) \\ \mathrm{C}(8) & 0.2565(7) & 0.3339(8) & 0.7231(5) & 2.5(2) \\ \mathrm{C}(9) & 0.1525(8) & 0.3744(8) & 0.6680(5) & 2.1(2) \\ \mathrm{C}(10) & 0.0286(7) & 0.4728(8) & 0.7110(5) & 1.9(2) \\ \mathrm{C}(11) & -0.0350(10) & 0.5825(9) & 0.8609(6) & 3.4(2) \\ \mathrm{C}(12) & 0.2745(9) & 0.380(1) & 0.8848(6) & 2.9(2) \\ \mathrm{C}(13) & 0.4097(8) & 0.248(1) & 0.6984(6) & 3.1(2) \\ \mathrm{C}(14) & 0.1677(10) & 0.339(1) & 0.5748(6) & 3.6(2) \\ \mathrm{C}(15) & -0.0965(8) & 0.5629(8) & 0.6683(6) & 2.6(2) \\ \mathrm{C}(16) & -0.1926(8) & 0.2971(8) & 0.5923(5) & 2.1(2) \\ \mathrm{C}(17) & -0.0872(10) & 0.201(1) & 0.5444(6) & 3.7(2) \\ \mathrm{C}(18) & -0.077(1) & 0.194(1) & 0.4547(7) & 4.5(3) \\ \mathrm{C}(19) & -0.173(1) & 0.290(1) & 0.4067(6) & 4.3(3) \\ \mathrm{C}(20) & -0.278(1) & 0.392(1) & 0.4512(8) & 5.3(4) \\ \mathrm{C}(21) & -0.290(1) & 0.395(1) & 0.5428(7) & 4.2(2) \\ \mathrm{C}(22) & -0.3636(7) & 0.4708(8) & 0.7861(6) & 2.2(2) \\ & & & \mathrm{S} 42 & \\ & & & \end{array}$




\begin{tabular}{|c|c|c|c|c|}
\hline $\mathrm{C}(23)$ & $-0.3570(9)$ & $0.508(1)$ & $0.8682(6)$ & $3.6(2)$ \\
\hline $\mathrm{C}(24)$ & $-0.4597(9)$ & $0.616(1)$ & $0.9066(7)$ & $3.5(2)$ \\
\hline $\mathrm{C}(25)$ & $-0.5764(10)$ & $0.6898(9)$ & $0.8665(7)$ & $3.9(2)$ \\
\hline$C(26)$ & $-0.588(1)$ & $0.653(1)$ & $0.7884(9)$ & $5.6(3)$ \\
\hline $\mathrm{C}(27)$ & $-0.483(1)$ & $0.539(1)$ & $0.7486(7)$ & $4.4(3)$ \\
\hline $\mathrm{C}(28)$ & $-0.2593(7)$ & $0.1412(7)$ & $0.7907(5)$ & $1.8(1)$ \\
\hline C(29) & $-0.2366(8)$ & $0.0291(8)$ & $0.7456(6)$ & $2.6(2)$ \\
\hline $\mathrm{C}(30)$ & $-0.2756(9)$ & $-0.0717(9)$ & $0.7856(7)$ & $3.5(2)$ \\
\hline $\mathrm{C}(31)$ & $-0.3407(8)$ & $-0.0661(9)$ & $0.8712(6)$ & $2.8(2)$ \\
\hline$C(32)$ & $-0.3680(8)$ & $0.0426(9)$ & $0.9179(6)$ & $2.9(2)$ \\
\hline $\mathrm{C}(33)$ & $-0.3264(8)$ & $0.1450(8)$ & $0.8770(5)$ & $2.5(2)$ \\
\hline $\mathrm{C}(34)$ & $-0.079(1)$ & $0.253(1)$ & $0.9984(6)$ & $4.1(3)$ \\
\hline $\mathrm{C}\left(35^{*}\right)$ & $0.438(3)$ & $0.147(3)$ & $0.408(2)$ & $5.2(5)$ \\
\hline$C\left(36^{*}\right)$ & $0.539(2)$ & $0.164(2)$ & $0.440(1)$ & $9.5(5)$ \\
\hline$C(37)$ & $0.388(3)$ & $-0.074(3)$ & $0.498(2)$ & $5.7(5)$ \\
\hline $\mathrm{C}(38)$ & $0.405(2)$ & $0.027(2)$ & $0.456(1)$ & $6.9(3)$ \\
\hline C(39) & $0.489(3)$ & $0.071(4)$ & $0.464(2)$ & $7.3(7)$ \\
\hline$C(100)$ & 0.1803 & 0.0337 & 0.8088 & 0.2000 \\
\hline $\mathrm{C}(101)$ & 0.1393 & 0.4128 & 0.7406 & 0.2000 \\
\hline $\mathrm{H}(1)$ & 0.0095 & 0.5716 & 0.9120 & 4.0907 \\
\hline $\mathrm{H}(2)$ & -0.0578 & 0.6703 & 0.8345 & 4.0907 \\
\hline $\mathrm{H}(3)$ & -0.1176 & 0.5668 & 0.8774 & 4.0907 \\
\hline $\mathrm{H}(4)$ & 0.2270 & 0.3515 & 0.9360 & 3.4831 \\
\hline $\mathrm{H}(5)$ & 0.3667 & 0.3153 & 0.8748 & 3.4831 \\
\hline $\mathrm{H}(6)$ & 0.2769 & 0.4621 & 0.8938 & 3.4831 \\
\hline $\mathrm{H}(7)$ & 0.4183 & 0.1649 & 0.6821 & 3.7112 \\
\hline $\mathrm{H}(8)$ & 0.4464 & 0.2913 & 0.6494 & 3.7112 \\
\hline $\mathrm{H}(9)$ & 0.4587 & 0.2350 & 0.7476 & 3.7112 \\
\hline $\mathrm{H}(10)$ & 0.1988 & 0.2449 & 0.5744 & 4.3460 \\
\hline $\mathrm{H}(11)$ & 0.0809 & 0.3803 & 0.5520 & 4.3460 \\
\hline $\mathrm{H}(12)$ & 0.2337 & 0.3669 & 0.5387 & 4.3460 \\
\hline $\mathrm{H}(13)$ & -0.0988 & 0.6504 & 0.6610 & 3.0784 \\
\hline $\mathrm{H}(14)$ & -0.0931 & 0.5335 & 0.6124 & 3.0784 \\
\hline $\mathrm{H}(15)$ & -0.1773 & 0.5626 & 0.7055 & 3.0784 \\
\hline $\mathrm{H}(16)$ & -0.1220 & 0.3209 & 1.0380 & 4.8618 \\
\hline $\mathrm{H}(17)$ & -0.1452 & 0.2218 & 0.9861 & 4.8618 \\
\hline $\mathrm{H}(18)$ & -0.0058 & 0.1812 & 1.0243 & 4.8618 \\
\hline H(19) & -0.0164 & 0.1348 & 0.5756 & 4.4144 \\
\hline $\mathrm{H}(20)$ & -0.0031 & 0.1227 & 0.4261 & 5.3658 \\
\hline $\mathrm{H}(21)$ & -0.1680 & 0.2865 & 0.3443 & 5.1952 \\
\hline $\mathrm{H}(22)$ & -0.3437 & 0.4627 & 0.4186 & 6.3542 \\
\hline $\mathrm{H}(23)$ & -0.3646 & 0.4641 & 0.5719 & 5.0556 \\
\hline $\mathrm{H}(24)$ & -0.2780 & 0.4576 & 0.8988 & 4.3714 \\
\hline $\mathrm{H}(25)$ & -0.4499 & 0.6394 & 0.9619 & 4.2268 \\
\hline $\mathrm{H}(26)$ & -0.6467 & 0.7649 & 0.8930 & 4.6770 \\
\hline $\mathrm{H}(27)$ & -0.6689 & 0.7020 & 0.7600 & 6.7016 \\
\hline
\end{tabular}




$\begin{array}{lrrrr}\mathrm{H}(28) & -0.4969 & 0.5132 & 0.6958 & 5.2471 \\ \mathrm{H}(29) & -0.1933 & 0.0232 & 0.6860 & 3.1637 \\ \mathrm{H}(30) & -0.2569 & -0.1458 & 0.7529 & 4.1429 \\ \mathrm{H}(31) & -0.3665 & -0.1367 & 0.8978 & 3.3697 \\ \mathrm{H}(32) & -0.4138 & 0.0487 & 0.9769 & 3.5390 \\ \mathrm{H}(33) & -0.3457 & 0.2194 & 0.9094 & 2.9894 \\ \mathrm{H}(34) & 0.0018 & -0.0005 & 0.8739 & 3.8887 \\ \mathrm{H}(35) & 0.0714 & 0.0113 & 0.7084 & 4.1891 \\ \mathrm{H}(36) & 0.1854 & 0.0188 & 0.9508 & 4.3120 \\ \mathrm{H}(37) & 0.3689 & 0.0453 & 0.8311 & 4.6402 \\ \mathrm{H}(38) & 0.3034 & 0.0354 & 0.6822 & 5.2194\end{array}$

$B_{\text {eq }}=8 / 3 \pi^{2}\left(U_{11}\left(a a^{*}\right)^{2}+U_{22}\left(b b^{*}\right)^{2}+U_{33}\left(c c^{*}\right)^{2}+2 U_{12}\left(a a^{*} b b^{*}\right) \cos \gamma+2 U_{13}\left(a a^{*} c c^{*}\right) \cos \beta+\right.$ $\left.2 \mathrm{U}_{23}\left(\mathrm{bb}^{*} \mathrm{cc} *\right) \cos \alpha\right)$

Table 33. Anisotropic Displacement Parameters for $\mathrm{CpCp} * \mathrm{Hf}\left(\mathrm{SnPh}_{3}\right) \mathrm{OMe} 0.5\left(\mathrm{C}_{7} \mathrm{H}_{8}\right)(\mathbf{1 0})$

$\begin{array}{lllllll}\text { atom } & \mathrm{U}_{11} & \mathrm{U}_{22} & \mathrm{U}_{33} & \mathrm{U}_{12} & \mathrm{U}_{13} & \mathrm{U}_{23} \\ \mathrm{Hf}(1) & 0.0182(2) & 0.0175(2) & 0.0248(2) & -0.0040(1) & -0.0009(1) & -0.0005(1) \\ \mathrm{Sn}(1) & 0.0196(3) & 0.0164(3) & 0.0240(3) & -0.0058(2) & 0.0001(2) & -0.0030(2) \\ \mathrm{O}(1) & 0.036(3) & 0.041(4) & 0.020(3) & -0.016(3) & 0.004(2) & -0.005(2) \\ \mathrm{C}(1) & 0.030(4) & 0.019(5) & 0.062(6) & -0.002(4) & -0.007(4) & -0.003(4) \\ \mathrm{C}(2) & 0.031(4) & 0.007(4) & 0.074(7) & 0.002(3) & -0.017(4) & 0.014(4) \\ \mathrm{C}(3) & 0.045(5) & 0.026(5) & 0.071(7) & -0.012(4) & -0.029(5) & 0.021(5) \\ \mathrm{C}(4) & 0.025(4) & 0.029(6) & 0.082(8) & -0.005(4) & -0.008(4) & 0.003(5) \\ \mathrm{C}(5) & 0.037(5) & 0.021(5) & 0.081(8) & 0.005(4) & 0.008(5) & -0.017(5) \\ \mathrm{C}(6) & 0.024(4) & 0.029(5) & 0.027(4) & -0.009(3) & -0.003(3) & -0.008(3) \\ \mathrm{C}(7) & 0.027(4) & 0.022(4) & 0.026(4) & -0.012(3) & -0.006(3) & 0.003(3) \\ \mathrm{C}(8) & 0.022(4) & 0.029(5) & 0.035(4) & -0.012(3) & 0.004(3) & -0.002(3) \\ \mathrm{C}(9) & 0.030(4) & 0.037(5) & 0.017(4) & -0.022(4) & 0.007(3) & -0.001(3) \\ \mathrm{C}(10) & 0.026(4) & 0.021(4) & 0.026(4) & -0.015(3) & -0.007(3) & 0.009(3) \\ \mathrm{C}(11) & 0.056(6) & 0.030(5) & 0.042(5) & -0.013(4) & -0.005(4) & -0.013(4) \\ \mathrm{C}(12) & 0.031(4) & 0.050(6) & 0.046(5) & -0.012(4) & -0.015(4) & -0.008(4) \\ \mathrm{C}(13) & 0.022(4) & 0.056(7) & 0.046(5) & -0.015(4) & 0.014(4) & -0.014(4) \\ \mathrm{C}(14) & 0.045(5) & 0.066(7) & 0.034(5) & -0.028(5) & 0.011(4) & -0.019(5) \\ \mathrm{C}(15) & 0.031(4) & 0.024(5) & 0.041(5) & -0.012(3) & -0.011(4) & 0.003(3) \\ \mathrm{C}(16) & 0.038(4) & 0.036(5) & 0.016(4) & -0.025(4) & 0.001(3) & 0.003(3) \\ \mathrm{C}(17) & 0.052(6) & 0.041(6) & 0.036(5) & -0.005(5) & -0.003(4) & -0.004(4) \\ \mathrm{C}(18) & 0.064(6) & 0.060(7) & 0.038(6) & -0.025(5) & 0.003(5) & -0.019(5) \\ \mathrm{C}(19) & 0.096(9) & 0.082(9) & 0.027(5) & -0.060(8) & -0.002(6) & -0.003(5) \\ \mathrm{C}(20) & 0.098(9) & 0.09(1) & 0.059(8) & -0.054(8) & -0.050(7) & 0.030(7) \\ \mathrm{C}(21) & 0.054(6) & 0.053(7) & 0.045(6) & -0.013(5) & -0.014(5) & 0.006(5) \\ \mathrm{C}(22) & 0.020(4) & 0.017(4) & 0.050(5) & -0.003(3) & 0.000(3) & -0.002(4) \\ \mathrm{C}(23) & 0.030(4) & 0.057(7) & 0.037(5) & -0.003(4) & 0.005(4) & -0.030(5) \\ \mathrm{C}(24) & 0.038(5) & 0.053(7) & 0.050(6) & -0.010(5) & 0.007(4) & -0.023(5) \\ \mathrm{C}(25) & 0.044(6) & 0.026(6) & 0.067(7) & -0.006(4) & 0.031(5) & -0.017(5)\end{array}$




$\begin{array}{lllllrr}\mathrm{C}(26) & 0.032(5) & 0.056(8) & 0.091(9) & 0.029(5) & -0.013(5) & -0.008(7) \\ \mathrm{C}(27) & 0.040(5) & 0.057(8) & 0.058(7) & 0.013(5) & -0.019(5) & -0.010(5) \\ \mathrm{C}(28) & 0.016(3) & 0.016(4) & 0.027(4) & -0.001(3) & -0.003(3) & -0.003(3) \\ \mathrm{C}(29) & 0.030(4) & 0.020(5) & 0.041(5) & -0.008(3) & 0.004(3) & -0.003(3) \\ \mathrm{C}(30) & 0.047(5) & 0.017(5) & 0.063(6) & -0.008(4) & -0.008(5) & -0.004(4) \\ \mathrm{C}(31) & 0.026(4) & 0.028(5) & 0.059(6) & -0.015(4) & -0.005(4) & 0.004(4) \\ \mathrm{C}(32) & 0.032(4) & 0.037(5) & 0.043(5) & -0.020(4) & -0.006(4) & 0.011(4) \\ \mathrm{C}(33) & 0.029(4) & 0.026(5) & 0.034(5) & -0.010(3) & -0.001(3) & -0.003(3) \\ \mathrm{C}(34) & 0.084(8) & 0.065(8) & 0.032(5) & -0.051(6) & -0.003(5) & 0.001(5)\end{array}$

The general temperature factor expression:

$\exp \left(-2 \pi^{2}\left(a^{*}{ }^{2} U_{11} h^{2}+b * 2 U_{22} k^{2}+c * 2 U_{33} l^{2}+2 a * b * U_{12} h k+2 a * c * U_{13} h l+2 b * c * U_{23} k l\right)\right)$

Table 34. Bond Lengths $(\AA)$ for $\mathrm{CpCp} * \mathrm{Hf}\left(\mathrm{SnPh}_{3}\right) \mathrm{OMe} \bullet 0.5\left(\mathrm{C}_{7} \mathrm{H}_{8}\right)(\mathbf{1 0})$

\begin{tabular}{|c|c|c|c|c|c|}
\hline atom & atom & distance & atom & atom & distance \\
\hline $\mathrm{Hf}(1)$ & $\operatorname{Sn}(1)$ & $2.9556(5)$ & $\mathrm{Hf}(1)$ & $\mathrm{O}(1)$ & $1.957(5)$ \\
\hline $\mathrm{Hf}(1)$ & $\mathrm{C}(1)$ & $2.547(9)$ & $\mathrm{Hf}(1)$ & $\mathrm{C}(2)$ & $2.567(9)$ \\
\hline $\mathrm{Hf}(1)$ & $\mathrm{C}(3)$ & $2.518(9)$ & $\mathrm{Hf}(1)$ & $\mathrm{C}(4)$ & $2.452(9)$ \\
\hline $\mathrm{Hf}(1)$ & $C(5)$ & $2.514(9)$ & $\mathrm{Hf}(1)$ & $C(6)$ & $2.518(8)$ \\
\hline $\mathrm{Hf}(1)$ & $C(7)$ & $2.468(7)$ & $\mathrm{Hf}(1)$ & $\mathrm{C}(8)$ & $2.532(7)$ \\
\hline $\mathrm{Hf}(1)$ & $\mathrm{C}(9)$ & $2.519(7)$ & $\mathrm{Hf}(1)$ & $\mathrm{C}(10)$ & $2.540(7)$ \\
\hline $\mathrm{Hf}(1)$ & $C(100)$ & $2.2190(3)$ & $\mathrm{Hf}(1)$ & $\mathrm{C}(101)$ & $2.2028(3)$ \\
\hline $\operatorname{Sn}(1)$ & $C(16)$ & $2.188(7)$ & $\operatorname{Sn}(1)$ & $\mathrm{C}(22)$ & $2.188(8)$ \\
\hline $\operatorname{Sn}(1)$ & $\mathrm{C}(28)$ & $2.174(7)$ & $\mathrm{O}(1)$ & $C(34)$ & $1.38(1)$ \\
\hline $\mathrm{C}(1)$ & $\mathrm{C}(2)$ & $1.40(1)$ & $\mathrm{C}(1)$ & $\mathrm{C}(5)$ & $1.42(1)$ \\
\hline$C(1)$ & $\mathrm{C}(100)$ & $1.199(9)$ & $\mathrm{C}(2)$ & $\mathrm{C}(3)$ & $1.40(1)$ \\
\hline$C(2)$ & $\mathrm{C}(100)$ & $1.188(9)$ & $\mathrm{C}(3)$ & $\mathrm{C}(4)$ & $1.41(1)$ \\
\hline$C(3)$ & $\mathrm{C}(100)$ & $1.20(1)$ & $\mathrm{C}(4)$ & $\mathrm{C}(5)$ & $1.39(2)$ \\
\hline $\mathrm{C}(4)$ & $\mathrm{C}(100)$ & $1.190(9)$ & $C(5)$ & $\mathrm{C}(100)$ & $1.19(1)$ \\
\hline$C(6)$ & $C(7)$ & $1.44(1)$ & $C(6)$ & $\mathrm{C}(10)$ & $1.43(1)$ \\
\hline$C(6)$ & $\mathrm{C}(11)$ & $1.49(1)$ & $C(6)$ & $\mathrm{C}(101)$ & $1.221(8)$ \\
\hline$C(7)$ & $\mathrm{C}(8)$ & $1.43(1)$ & $C(7)$ & $\mathrm{C}(12)$ & $1.51(1)$ \\
\hline$C(7)$ & $\mathrm{C}(101)$ & $1.217(7)$ & $\mathrm{C}(8)$ & $\mathrm{C}(9)$ & $1.39(1)$ \\
\hline $\mathrm{C}(8)$ & $\mathrm{C}(13)$ & $1.53(1)$ & $\mathrm{C}(8)$ & $\mathrm{C}(101)$ & $1.206(7)$ \\
\hline $\mathrm{C}(9)$ & $\mathrm{C}(10)$ & $1.45(1)$ & $\mathrm{C}(9)$ & $\mathrm{C}(14)$ & $1.50(1)$ \\
\hline $\mathrm{C}(9)$ & $\mathrm{C}(101)$ & $1.207(7)$ & $\mathrm{C}(10)$ & $C(15)$ & $1.489(10)$ \\
\hline$C(10)$ & $\mathrm{C}(101)$ & $1.219(7)$ & $C(16)$ & $\mathrm{C}(17)$ & $1.38(1)$ \\
\hline$C(16)$ & $\mathrm{C}(21)$ & $1.39(1)$ & $\mathrm{C}(17)$ & $\mathrm{C}(18)$ & $1.37(1)$ \\
\hline $\mathrm{C}(18)$ & $\mathrm{C}(19)$ & $1.36(2)$ & $C(19)$ & $C(20)$ & $1.38(2)$ \\
\hline$C(20)$ & $\mathrm{C}(21)$ & $1.39(2)$ & $C(22)$ & $\mathrm{C}(23)$ & $1.39(1)$ \\
\hline$C(22)$ & $\mathrm{C}(27)$ & $1.36(1)$ & $\mathrm{C}(23)$ & $\mathrm{C}(24)$ & $1.37(1)$ \\
\hline$C(24)$ & $C(25)$ & $1.37(1)$ & $C(25)$ & $C(26)$ & $1.35(2)$ \\
\hline$C(26)$ & $\mathrm{C}(27)$ & $1.43(2)$ & $\mathrm{C}(28)$ & $C(29)$ & $1.41(1)$ \\
\hline$C(28)$ & $\mathrm{C}(33)$ & $1.39(1)$ & $\mathrm{C}(29)$ & $C(30)$ & $1.38(1)$ \\
\hline
\end{tabular}




$\begin{array}{llllll}\mathrm{C}(30) & \mathrm{C}(31) & 1.37(1) & \mathrm{C}(31) & \mathrm{C}(32) & 1.38(1) \\ \mathrm{C}(32) & \mathrm{C}(33) & 1.42(1) & \mathrm{C}\left(35^{*}\right) & \mathrm{C}\left(36^{*}\right) & 1.34(3) \\ \mathrm{C}\left(35^{*}\right) & \mathrm{C}(38) & 1.57(3) & \mathrm{C}\left(35^{*}\right) & \mathrm{C}(39) & 1.15(4) \\ \mathrm{C}\left(36^{*}\right) & \mathrm{C}(37) & 1.36(3) & \mathrm{C}\left(36^{*}\right) & \mathrm{C}(39) & 1.32(4) \\ \mathrm{C}(37) & \mathrm{C}(38) & 1.28(3) & \mathrm{C}(37) & \mathrm{C}(39) & 1.52(4) \\ \mathrm{C}(38) & \mathrm{C}(39) & 1.21(4) & \mathrm{C}(38) & \mathrm{C}(39) & 1.75(3) \\ \mathrm{C}(39) & \mathrm{C}(39) & 1.76(7) & & & \end{array}$

Table 35. Bond Angles $\left({ }^{\circ}\right)$ for $\mathrm{CpCp} * \mathrm{Hf}\left(\mathrm{SnPh}_{3}\right) \mathrm{OMe} \bullet 0.5\left(\mathrm{C}_{7} \mathrm{H}_{8}\right)(\mathbf{1 0})$

\begin{tabular}{|c|c|c|c|c|c|c|c|}
\hline atom & atom & atom & angle & atom & atom & atom & angle \\
\hline $\operatorname{Sn}(1)$ & $\operatorname{Hf}(1)$ & $\mathrm{O}(1)$ & $90.6(2)$ & $\operatorname{Sn}(1)$ & $\operatorname{Hf}(1)$ & $\mathrm{C}(1)$ & $76.4(2)$ \\
\hline $\operatorname{Sn}(1)$ & $\operatorname{Hf}(1)$ & $C(2)$ & $82.7(2)$ & $\operatorname{Sn}(1)$ & $\operatorname{Hf}(1)$ & $C(3)$ & $114.0(2)$ \\
\hline $\operatorname{Sn}(1)$ & $\mathrm{Hf}(1)$ & $\mathrm{C}(4)$ & $130.2(2)$ & $\operatorname{Sn}(1)$ & $\operatorname{Hf}(1)$ & $\mathrm{C}(5)$ & 104.1(3) \\
\hline $\operatorname{Sn}(1)$ & $\operatorname{Hf}(1)$ & $C(6)$ & $101.4(2)$ & $\operatorname{Sn}(1)$ & $\operatorname{Hf}(1)$ & $C(7)$ & $133.4(2)$ \\
\hline $\operatorname{Sn}(1)$ & $\operatorname{Hf}(1)$ & $\mathrm{C}(8)$ & $125.6(2)$ & $\operatorname{Sn}(1)$ & $\operatorname{Hf}(1)$ & $C(9)$ & $93.6(2)$ \\
\hline $\operatorname{Sn}(1)$ & $\mathrm{Hf}(1)$ & $\mathrm{C}(10)$ & $79.5(2)$ & $\mathrm{O}(1)$ & $\operatorname{Hf}(1)$ & $\mathrm{C}(1)$ & $118.6(3)$ \\
\hline $\mathrm{O}(1)$ & $\operatorname{Hf}(1)$ & $\mathrm{C}(2)$ & $87.9(3)$ & $\mathrm{O}(1)$ & $\operatorname{Hf}(1)$ & $C(3)$ & $80.9(3)$ \\
\hline $\mathrm{O}(1)$ & $\mathrm{Hf}(1)$ & $\mathrm{C}(4)$ & 108.1(3) & $\mathrm{O}(1)$ & $\operatorname{Hf}(1)$ & $\mathrm{C}(5)$ & $134.7(3)$ \\
\hline $\mathrm{O}(1)$ & $\operatorname{Hf}(1)$ & $C(6)$ & $80.6(3)$ & $\mathrm{O}(1)$ & $\operatorname{Hf}(1)$ & $C(7)$ & $91.4(2)$ \\
\hline $\mathrm{O}(1)$ & $\mathrm{Hf}(1)$ & $\mathrm{C}(8)$ & $124.6(3)$ & $\mathrm{O}(1)$ & $\operatorname{Hf}(1)$ & $\mathrm{C}(9)$ & $135.1(3)$ \\
\hline $\mathrm{O}(1)$ & $\mathrm{Hf}(1)$ & $\mathrm{C}(10)$ & $104.9(3)$ & $\mathrm{C}(1)$ & $\operatorname{Hf}(1)$ & $\mathrm{C}(2)$ & $31.7(3)$ \\
\hline $\mathrm{C}(1)$ & $\operatorname{Hf}(1)$ & $C(3)$ & $53.5(3)$ & $C(1)$ & $\operatorname{Hf}(1)$ & $C(4)$ & $54.0(3)$ \\
\hline$C(1)$ & $\operatorname{Hf}(1)$ & $\mathrm{C}(5)$ & $32.5(3)$ & $C(1)$ & $\operatorname{Hf}(1)$ & $C(6)$ & $160.4(3)$ \\
\hline$C(1)$ & $\operatorname{Hf}(1)$ & $C(7)$ & $139.6(3)$ & $C(1)$ & $\operatorname{Hf}(1)$ & $C(8)$ & $110.6(3)$ \\
\hline $\mathrm{C}(1)$ & $\operatorname{Hf}(1)$ & $\mathrm{C}(9)$ & $105.8(3)$ & $\mathrm{C}(1)$ & $\operatorname{Hf}(1)$ & $C(10)$ & $129.8(3)$ \\
\hline$C(2)$ & $\mathrm{Hf}(1)$ & $C(3)$ & $32.0(3)$ & $C(2)$ & $\operatorname{Hf}(1)$ & $C(4)$ & $53.6(3)$ \\
\hline$C(2)$ & $\mathrm{Hf}(1)$ & $C(5)$ & $53.0(3)$ & $\mathrm{C}(2)$ & $\operatorname{Hf}(1)$ & $\mathrm{C}(6)$ & $167.7(3)$ \\
\hline$C(2)$ & $\operatorname{Hf}(1)$ & $C(7)$ & $143.9(2)$ & $C(2)$ & $\operatorname{Hf}(1)$ & $\mathrm{C}(8)$ & $131.9(3)$ \\
\hline$C(2)$ & $\operatorname{Hf}(1)$ & $\mathrm{C}(9)$ & $137.0(3)$ & $C(2)$ & $\operatorname{Hf}(1)$ & $C(10)$ & $158.1(3)$ \\
\hline$C(3)$ & $\operatorname{Hf}(1)$ & $\mathrm{C}(4)$ & $33.0(3)$ & $C(3)$ & $\operatorname{Hf}(1)$ & $C(5)$ & $53.8(4)$ \\
\hline$C(3)$ & $\operatorname{Hf}(1)$ & $C(6)$ & $140.0(3)$ & $C(3)$ & $\operatorname{Hf}(1)$ & $C(7)$ & $112.3(3)$ \\
\hline$C(3)$ & $\mathrm{Hf}(1)$ & $\mathrm{C}(8)$ & $111.8(3)$ & $\mathrm{C}(3)$ & $\operatorname{Hf}(1)$ & $\mathrm{C}(9)$ & $135.7(3)$ \\
\hline$C(3)$ & $\operatorname{Hf}(1)$ & $\mathrm{C}(10)$ & $165.6(3)$ & $C(4)$ & $\operatorname{Hf}(1)$ & $C(5)$ & $32.5(4)$ \\
\hline C(4) & $\mathrm{Hf}(1)$ & $C(6)$ & $126.5(3)$ & $\mathrm{C}(4)$ & $\operatorname{Hf}(1)$ & $C(7)$ & $92.9(3)$ \\
\hline$C(4)$ & $\operatorname{Hf}(1)$ & $\mathrm{C}(8)$ & $80.9(3)$ & $C(4)$ & $\operatorname{Hf}(1)$ & $C(9)$ & $102.7(3)$ \\
\hline C(4) & $\operatorname{Hf}(1)$ & $\mathrm{C}(10)$ & $134.0(3)$ & $C(5)$ & $\operatorname{Hf}(1)$ & $C(6)$ & $135.2(3)$ \\
\hline$C(5)$ & $\operatorname{Hf}(1)$ & $C(7)$ & 107.1(3) & $C(5)$ & $\operatorname{Hf}(1)$ & $C(8)$ & $80.7(3)$ \\
\hline$C(5)$ & $\operatorname{Hf}(1)$ & $\mathrm{C}(9)$ & $87.3(3)$ & $C(5)$ & $\operatorname{Hf}(1)$ & $C(10)$ & $119.8(3)$ \\
\hline C(6) & $\mathrm{Hf}(1)$ & $C(7)$ & $33.6(2)$ & $C(6)$ & $\operatorname{Hf}(1)$ & $\mathrm{C}(8)$ & $54.5(3)$ \\
\hline $\mathrm{C}(6)$ & $\mathrm{Hf}(1)$ & $\mathrm{C}(9)$ & $54.7(3)$ & $C(6)$ & $\operatorname{Hf}(1)$ & $C(10)$ & $32.8(2)$ \\
\hline$C(7)$ & $\mathrm{Hf}(1)$ & $C(8)$ & $33.2(3)$ & $C(7)$ & $\operatorname{Hf}(1)$ & $\mathrm{C}(9)$ & $54.8(2)$ \\
\hline$C(7)$ & $\mathrm{Hf}(1)$ & $\mathrm{C}(10)$ & $55.1(2)$ & $C(8)$ & $\operatorname{Hf}(1)$ & $C(9)$ & $31.9(2)$ \\
\hline C(8) & $\mathrm{Hf}(1)$ & $\mathrm{C}(10)$ & $54.0(2)$ & $\mathrm{C}(9)$ & $\operatorname{Hf}(1)$ & $C(10)$ & $33.2(3)$ \\
\hline $\operatorname{Hf}(1)$ & $\operatorname{Sn}(1)$ & $C(16)$ & $119.7(2)$ & $\operatorname{Hf}(1)$ & $\operatorname{Sn}(1)$ & $C(22)$ & $112.9(2)$ \\
\hline $\operatorname{Hf}(1)$ & $\operatorname{Sn}(1)$ & $\mathrm{C}(28)$ & $110.3(2)$ & $C(16)$ & $\operatorname{Sn}(1)$ & $C(22)$ & $108.9(3)$ \\
\hline
\end{tabular}




\begin{tabular}{|c|c|c|c|c|c|c|c|}
\hline$C(16)$ & $\operatorname{Sn}(1)$ & $\mathrm{C}(28)$ & $102.6(3)$ & $C(22)$ & $\operatorname{Sn}(1)$ & $\mathrm{C}(28)$ & $100.2(3)$ \\
\hline $\mathrm{Hf}(1)$ & $\mathrm{O}(1)$ & $C(34)$ & $143.3(6)$ & $\operatorname{Hf}(1)$ & $\mathrm{C}(1)$ & $\mathrm{C}(2)$ & $74.9(5)$ \\
\hline $\mathrm{Hf}(1)$ & $\mathrm{C}(1)$ & $C(5)$ & $72.4(5)$ & $C(2)$ & $\mathrm{C}(1)$ & $C(5)$ & $107.3(8)$ \\
\hline $\mathrm{Hf}(1)$ & $\mathrm{C}(2)$ & $\mathrm{C}(1)$ & $73.4(5)$ & $\operatorname{Hf}(1)$ & $\mathrm{C}(2)$ & $\mathrm{C}(3)$ & $72.1(5)$ \\
\hline $\mathrm{C}(1)$ & $\mathrm{C}(2)$ & $C(3)$ & $109.1(8)$ & $\operatorname{Hf}(1)$ & $\mathrm{C}(3)$ & $\mathrm{C}(2)$ & $75.9(5)$ \\
\hline $\mathrm{Hf}(1)$ & $\mathrm{C}(3)$ & $\mathrm{C}(4)$ & $70.9(5)$ & $\mathrm{C}(2)$ & $\mathrm{C}(3)$ & $\mathrm{C}(4)$ & $106.9(9)$ \\
\hline $\mathrm{Hf}(1)$ & $\mathrm{C}(4)$ & $\mathrm{C}(3)$ & $76.0(5)$ & $\operatorname{Hf}(1)$ & $C(4)$ & $C(5)$ & $76.2(5)$ \\
\hline$C(3)$ & $C(4)$ & $C(5)$ & $108.6(9)$ & $\operatorname{Hf}(1)$ & $\mathrm{C}(5)$ & $\mathrm{C}(1)$ & $75.1(5)$ \\
\hline $\mathrm{Hf}(1)$ & $C(5)$ & $C(4)$ & $71.3(6)$ & $\mathrm{C}(1)$ & $\mathrm{C}(5)$ & $\mathrm{C}(4)$ & $108.0(9)$ \\
\hline $\mathrm{Hf}(1)$ & $C(6)$ & $C(7)$ & $71.3(4)$ & $\operatorname{Hf}(1)$ & $\mathrm{C}(6)$ & $\mathrm{C}(10)$ & $74.5(4)$ \\
\hline $\mathrm{Hf}(1)$ & $\mathrm{C}(6)$ & $\mathrm{C}(11)$ & $122.2(6)$ & $\mathrm{C}(7)$ & $\mathrm{C}(6)$ & $\mathrm{C}(10)$ & $107.8(7)$ \\
\hline$C(7)$ & $\mathrm{C}(6)$ & $\mathrm{C}(11)$ & $126.0(7)$ & $C(10)$ & $\mathrm{C}(6)$ & $\mathrm{C}(11)$ & $126.2(7)$ \\
\hline $\mathrm{Hf}(1)$ & $\mathrm{C}(7)$ & $C(6)$ & $75.1(4)$ & $\operatorname{Hf}(1)$ & $\mathrm{C}(7)$ & $\mathrm{C}(8)$ & $75.8(4)$ \\
\hline $\mathrm{Hf}(1)$ & $C(7)$ & $\mathrm{C}(12)$ & $118.2(6)$ & $\mathrm{C}(6)$ & $C(7)$ & $\mathrm{C}(8)$ & $107.2(6)$ \\
\hline $\mathrm{C}(6)$ & $C(7)$ & $C(12)$ & $125.2(7)$ & $\mathrm{C}(8)$ & $C(7)$ & $\mathrm{C}(12)$ & $127.5(7)$ \\
\hline $\mathrm{Hf}(1)$ & $\mathrm{C}(8)$ & $C(7)$ & $70.9(4)$ & $\operatorname{Hf}(1)$ & $\mathrm{C}(8)$ & $\mathrm{C}(9)$ & $73.5(4)$ \\
\hline $\mathrm{Hf}(1)$ & $\mathrm{C}(8)$ & $\mathrm{C}(13)$ & $126.2(6)$ & $C(7)$ & $\mathrm{C}(8)$ & $\mathrm{C}(9)$ & $109.0(6)$ \\
\hline$C(7)$ & $\mathrm{C}(8)$ & $\mathrm{C}(13)$ & $124.5(7)$ & $\mathrm{C}(9)$ & $\mathrm{C}(8)$ & $\mathrm{C}(13)$ & $126.2(7)$ \\
\hline $\mathrm{Hf}(1)$ & $\mathrm{C}(9)$ & $C(8)$ & $74.6(4)$ & $\operatorname{Hf}(1)$ & $\mathrm{C}(9)$ & $\mathrm{C}(10)$ & $74.2(4)$ \\
\hline $\mathrm{Hf}(1)$ & $\mathrm{C}(9)$ & $\mathrm{C}(14)$ & $123.6(6)$ & $\mathrm{C}(8)$ & $\mathrm{C}(9)$ & $\mathrm{C}(10)$ & $108.5(7)$ \\
\hline $\mathrm{C}(8)$ & $\mathrm{C}(9)$ & $C(14)$ & $126.8(7)$ & $C(10)$ & $\mathrm{C}(9)$ & $\mathrm{C}(14)$ & $124.2(7)$ \\
\hline $\mathrm{Hf}(1)$ & $C(10)$ & $C(6)$ & $72.8(4)$ & $\operatorname{Hf}(1)$ & $\mathrm{C}(10)$ & $\mathrm{C}(9)$ & $72.5(4)$ \\
\hline $\mathrm{Hf}(1)$ & $C(10)$ & $\mathrm{C}(15)$ & $129.7(5)$ & $C(6)$ & $\mathrm{C}(10)$ & $\mathrm{C}(9)$ & $107.3(6)$ \\
\hline$C(6)$ & $C(10)$ & $C(15)$ & $125.3(7)$ & $\mathrm{C}(9)$ & $\mathrm{C}(10)$ & $C(15)$ & $126.2(7)$ \\
\hline $\operatorname{Sn}(1)$ & $C(16)$ & $\mathrm{C}(17)$ & $119.5(6)$ & $\operatorname{Sn}(1)$ & $C(16)$ & $\mathrm{C}(21)$ & $124.7(7)$ \\
\hline$C(17)$ & $C(16)$ & $\mathrm{C}(21)$ & $115.6(8)$ & $C(16)$ & $\mathrm{C}(17)$ & $\mathrm{C}(18)$ & $124.4(9)$ \\
\hline$C(17)$ & $\mathrm{C}(18)$ & $\mathrm{C}(19)$ & $119.6(10)$ & $\mathrm{C}(18)$ & $\mathrm{C}(19)$ & $\mathrm{C}(20)$ & 118.2(9) \\
\hline C(19) & $\mathrm{C}(20)$ & $\mathrm{C}(21)$ & $121(1)$ & $C(16)$ & $\mathrm{C}(21)$ & $\mathrm{C}(20)$ & $120(1)$ \\
\hline $\operatorname{Sn}(1)$ & $C(22)$ & $\mathrm{C}(23)$ & $117.0(6)$ & $\operatorname{Sn}(1)$ & $\mathrm{C}(22)$ & $\mathrm{C}(27)$ & $126.1(7)$ \\
\hline$C(23)$ & $\mathrm{C}(22)$ & $\mathrm{C}(27)$ & $116.7(8)$ & $C(22)$ & $\mathrm{C}(23)$ & $\mathrm{C}(24)$ & $122.4(8)$ \\
\hline$C(23)$ & $\mathrm{C}(24)$ & $C(25)$ & $120.9(9)$ & $C(24)$ & $\mathrm{C}(25)$ & $C(26)$ & $118.2(8)$ \\
\hline$C(25)$ & $C(26)$ & $\mathrm{C}(27)$ & $121.3(9)$ & $C(22)$ & $\mathrm{C}(27)$ & $C(26)$ & $120(1)$ \\
\hline $\operatorname{Sn}(1)$ & $\mathrm{C}(28)$ & $\mathrm{C}(29)$ & $124.0(6)$ & $\operatorname{Sn}(1)$ & $\mathrm{C}(28)$ & $\mathrm{C}(33)$ & $120.1(5)$ \\
\hline $\mathrm{C}(29)$ & $\mathrm{C}(28)$ & $\mathrm{C}(33)$ & $115.9(7)$ & $C(28)$ & $\mathrm{C}(29)$ & $C(30)$ & $122.0(8)$ \\
\hline C(29) & $C(30)$ & $\mathrm{C}(31)$ & $121.0(9)$ & $C(30)$ & $\mathrm{C}(31)$ & $\mathrm{C}(32)$ & $119.4(8)$ \\
\hline $\mathrm{C}(31)$ & $C(32)$ & $\mathrm{C}(33)$ & $119.4(8)$ & $\mathrm{C}(28)$ & $\mathrm{C}(33)$ & $C(32)$ & $122.3(8)$ \\
\hline $\mathrm{C}\left(36^{*}\right)$ & $\mathrm{C}\left(35^{*}\right)$ & $\mathrm{C}(38)$ & $113(2)$ & $\mathrm{C}\left(36^{*}\right)$ & $\mathrm{C}\left(35^{*}\right)$ & $C(39)$ & $63(2)$ \\
\hline $\mathrm{C}(38)$ & $\mathrm{C}\left(35^{*}\right)$ & $C(39)$ & $49(2)$ & $\mathrm{C}\left(35^{*}\right)$ & $\mathrm{C}\left(36^{*}\right)$ & $\mathrm{C}(37)$ & $120(2)$ \\
\hline $\mathrm{C}\left(35^{*}\right)$ & $\mathrm{C}\left(36^{*}\right)$ & $C(39)$ & $51(1)$ & $C(37)$ & $\mathrm{C}\left(36^{*}\right)$ & $\mathrm{C}(39)$ & $69(2)$ \\
\hline$C\left(36^{*}\right)$ & $\mathrm{C}(37)$ & $\mathrm{C}(38)$ & $130(2)$ & $\mathrm{C}\left(36^{*}\right)$ & $\mathrm{C}(37)$ & $\mathrm{C}(39)$ & $54(1)$ \\
\hline $\mathrm{C}(38)$ & $C(37)$ & $C(39)$ & $76(2)$ & $\mathrm{C}\left(35^{*}\right)$ & $\mathrm{C}(38)$ & $\mathrm{C}(37)$ & $174(1)$ \\
\hline $\mathrm{C}\left(35^{*}\right)$ & $\mathrm{C}(38)$ & $C(39)$ & $46(1)$ & $\mathrm{C}\left(35^{*}\right)$ & $\mathrm{C}(38)$ & $\mathrm{C}(39)$ & $117(1)$ \\
\hline $\mathrm{C}(37)$ & $\mathrm{C}(38)$ & $\mathrm{C}(39)$ & $128(2)$ & $\mathrm{C}(37)$ & $\mathrm{C}(38)$ & C(39) & $58(1)$ \\
\hline C(39) & $\mathrm{C}(38)$ & $C(39)$ & $70(2)$ & $\mathrm{C}\left(35^{*}\right)$ & $C(39)$ & $\mathrm{C}\left(36^{*}\right)$ & $65(2)$ \\
\hline $\mathrm{C}\left(35^{*}\right)$ & $C(39)$ & $C(37)$ & $121(3)$ & $\mathrm{C}\left(35^{*}\right)$ & $C(39)$ & $\mathrm{C}(38)$ & $83(2)$ \\
\hline $\mathrm{C}\left(35^{*}\right)$ & $C(39)$ & $\mathrm{C}(38)$ & $166(3)$ & $\mathrm{C}\left(35^{*}\right)$ & $\mathrm{C}(39)$ & $C(39)$ & $152(4)$ \\
\hline
\end{tabular}




$\begin{array}{llllllll}\mathrm{C}\left(36^{*}\right) & \mathrm{C}(39) & \mathrm{C}(37) & 56(1) & \mathrm{C}\left(36^{*}\right) & \mathrm{C}(39) & \mathrm{C}(38) & 148(3) \\ \mathrm{C}\left(36^{*}\right) & \mathrm{C}(39) & \mathrm{C}(38) & 101(2) & \mathrm{C}\left(36^{*}\right) & \mathrm{C}(39) & \mathrm{C}(39) & 142(3) \\ \mathrm{C}(37) & \mathrm{C}(39) & \mathrm{C}(38) & 154(3) & \mathrm{C}(37) & \mathrm{C}(39) & \mathrm{C}(38) & 45(1) \\ \mathrm{C}(37) & \mathrm{C}(39) & \mathrm{C}(39) & 85(2) & \mathrm{C}(38) & \mathrm{C}(39) & \mathrm{C}(38) & 109(2) \\ \mathrm{C}(38) & \mathrm{C}(39) & \mathrm{C}(39) & 69(2) & \mathrm{C}(38) & \mathrm{C}(39) & \mathrm{C}(39) & 40(1)\end{array}$

Table 36. Observed first-order rate constants for the disappearance of $\mathbf{4}$ at different temperatures.

\begin{tabular}{|c|c|c|}
\hline Initial Concentration $(\mu \mathrm{M})$ & Rate constant $\left(\mathrm{s}^{-1}\right)$ & Temperature $\left({ }^{\circ} \mathrm{C}\right)$ \\
\hline 15.0 & $1.4 \times 10^{-6}$ & 70 \\
\hline 15.0 & $1.5 \times 10^{-6}$ & 70 \\
\hline 15.0 & $5.6 \times 10^{-6}$ & 85 \\
\hline 15.0 & $5.7 \times 10^{-6}$ & 100 \\
\hline 15.0 & $2.3 \times 10^{-5}$ & 100 \\
\hline 15.0 & $2.2 \times 10^{-5}$ & 115 \\
\hline 15.0 & $1.0 \times 10^{-4}$ & 115 \\
\hline 15.0 & $1.1 \times 10^{-4}$ & \\
\hline
\end{tabular}

Table 37. Observed first-order rate constants for the disappearance of various Hf-Sn species.

\begin{tabular}{|c|c|c|c|}
\hline Compound & $\begin{array}{c}\text { Initial Concentration } \\
(\mu \mathrm{M})\end{array}$ & Rate Constant $\left(\mathrm{s}^{-1}\right)$ & $\begin{array}{c}\text { Temperature } \\
\left({ }^{\circ} \mathrm{C}\right)\end{array}$ \\
\hline $\mathrm{CpCp} * \mathrm{Hf}\left(\mathrm{SnPh}_{3}\right) \mathrm{OMe}(\mathbf{1 0})_{\mathrm{CpCp}}^{*} \mathrm{Hf}\left(\mathrm{SnPh}_{3}\right) \mathrm{NMe}_{2}(\mathbf{8})$ & 14.9 & $8.8 \times 10^{-6}$ & 100 \\
\hline $\mathrm{CpCp} * \mathrm{Hf}\left(\mathrm{SnPh}_{3}\right) \mathrm{Me} \mathrm{(9)}$ & 14.9 & $4.3 \times 10^{-7}$ & 115 \\
\hline $\mathrm{CpCp} * \mathrm{Hf}\left(\mathrm{SnPh}_{3}\right) \mathrm{Me}(\mathbf{9})^{-7}$ & 14.7 & $9.6 \times 10^{-5}$ & 70 \\
\hline$\left[\mathrm{Me}_{2} \mathrm{C}\left(\mathrm{C}_{5} \mathrm{H}_{4}\right)_{2}\right] \mathrm{Hf}\left(\mathrm{SnPh}_{3}\right) \mathrm{NMe}_{2}(\mathbf{2})$ & 14.5 & $5.6 \times 10^{-6}$ & 45 \\
\hline
\end{tabular}

Table 38. Observed first-order rate constants for the disappearance of $\left[\mathrm{CpCp} * \mathrm{Hf}\left[\mathrm{Sn}\left(p-\mathrm{XC}_{6} \mathrm{H}_{4}\right)_{3}\right] \mathrm{Cl}\right]$ at $100{ }^{\circ} \mathrm{C}$.

\begin{tabular}{|c|c|c|c|}
\hline $\mathrm{X}$ & Substituent Constant $(\sigma)$ & Initial Concentration $(\mu \mathrm{M})$ & Rate Constant $\left(\mathrm{s}^{-1}\right)$ \\
\hline $\mathrm{H}(\mathbf{5})$ & 0 & 15.2 & $2.9 \times 10^{-5}$ \\
\hline $\mathrm{OMe}(\mathbf{1 9})$ & -0.268 & 14.1 & $1.8 \times 10^{-4}$ \\
\hline $\mathrm{F}(\mathbf{2 0})$ & 0.062 & 6.7 & $2.9 \times 10^{-5}$ \\
\hline $\mathrm{CF}_{3}(\mathbf{2 1})$ & 0.54 & 15.0 & $3.1 \times 10^{-6}$ \\
\hline
\end{tabular}


Figure 1. ORTEP diagram of $\left[\mathrm{Me}_{2} \mathrm{C}\left(\mathrm{C}_{5} \mathrm{H}_{4}\right)_{2}\right] \mathrm{Hf}\left(\mathrm{SnPh}_{3}\right) \mathrm{NMe}_{2}(2)$. The hydrogen atoms were removed for clarity. Atoms are shown as $50 \%$ probability ellipsoids.

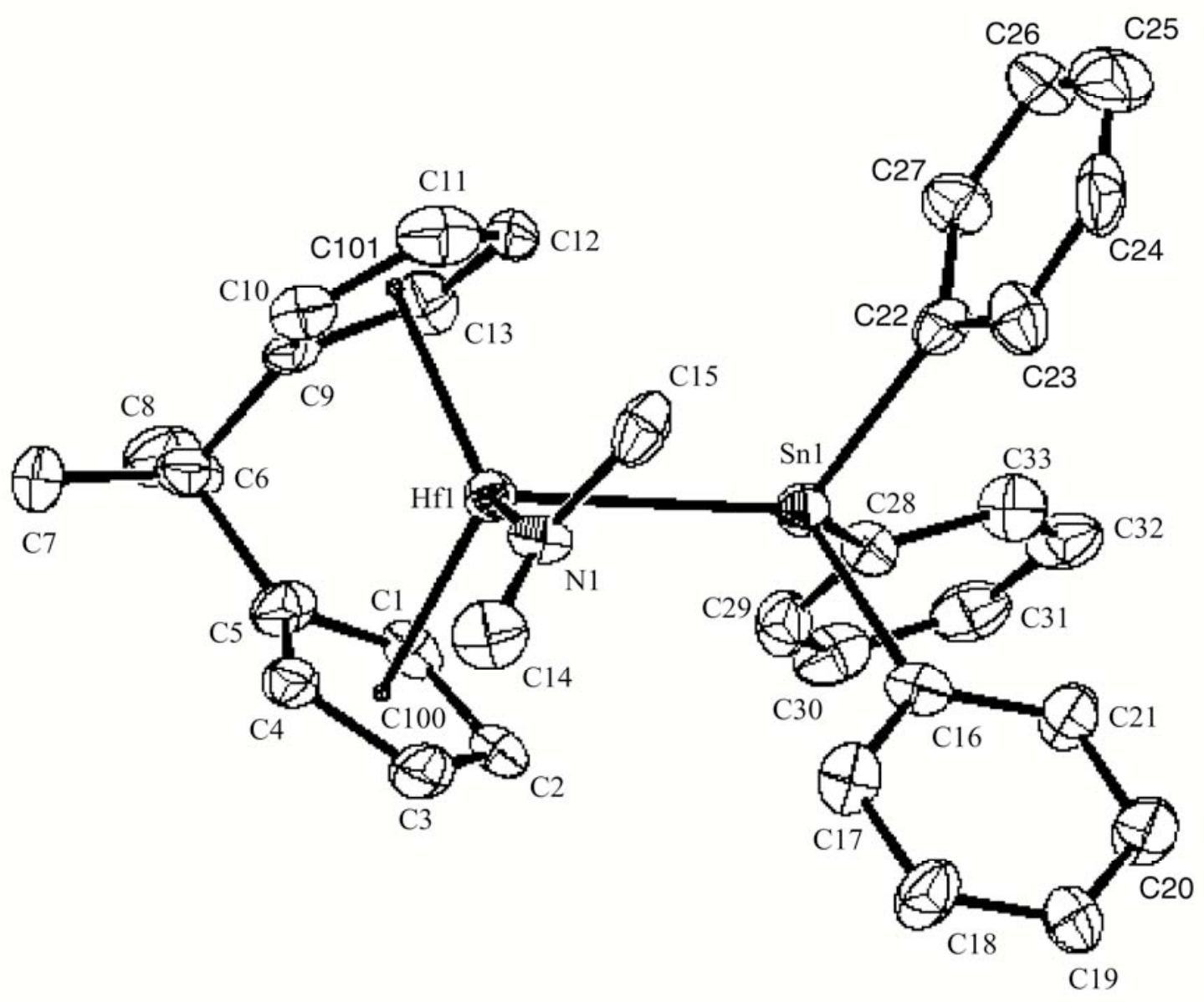


Figure 2. ORTEP diagram of $\mathrm{CpCp} * \mathrm{Hf}\left(\mathrm{SnPh}_{3}\right) \mathrm{Cl}(4)$. The hydrogen atoms were removed for clarity. Atoms are shown as $50 \%$ probability ellipsoids.

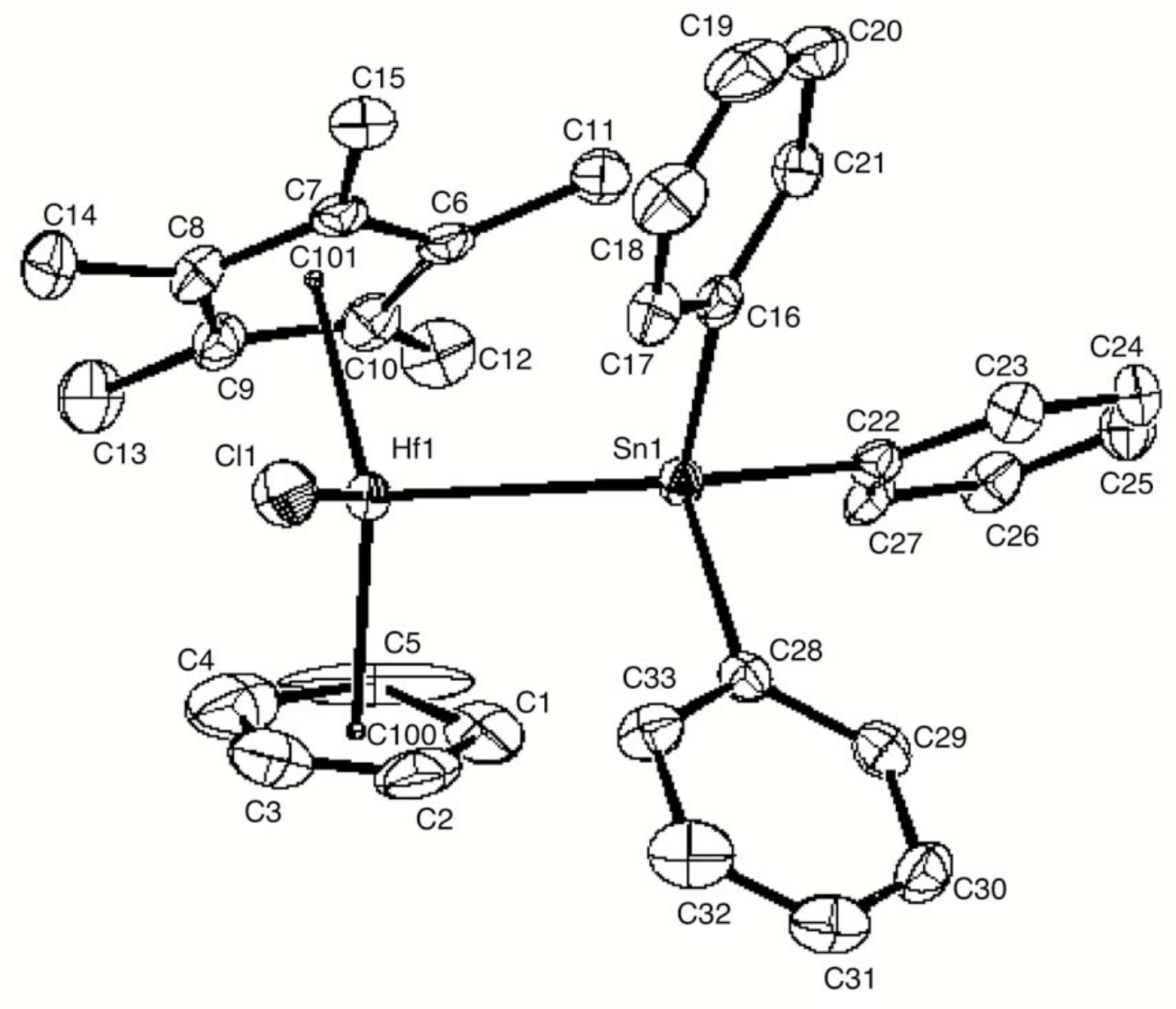


Figure 3. ORTEP diagram of $\mathrm{CpCp} * \mathrm{Hf}\left(\mathrm{SnPh}_{3}\right) \mathrm{Me} \cdot 0.5\left(\mathrm{C}_{5} \mathrm{H}_{12}\right)\left(\mathbf{9} \boldsymbol{0} \mathbf{0 . 5}\left(\mathbf{C}_{\mathbf{5}} \mathbf{H}_{\mathbf{1 2}}\right)\right)$. The hydrogen atoms and the $\mathrm{C}_{5} \mathrm{H}_{12}$ molecule were removed for clarity. Atoms are shown as 50\% probability ellipsoids.

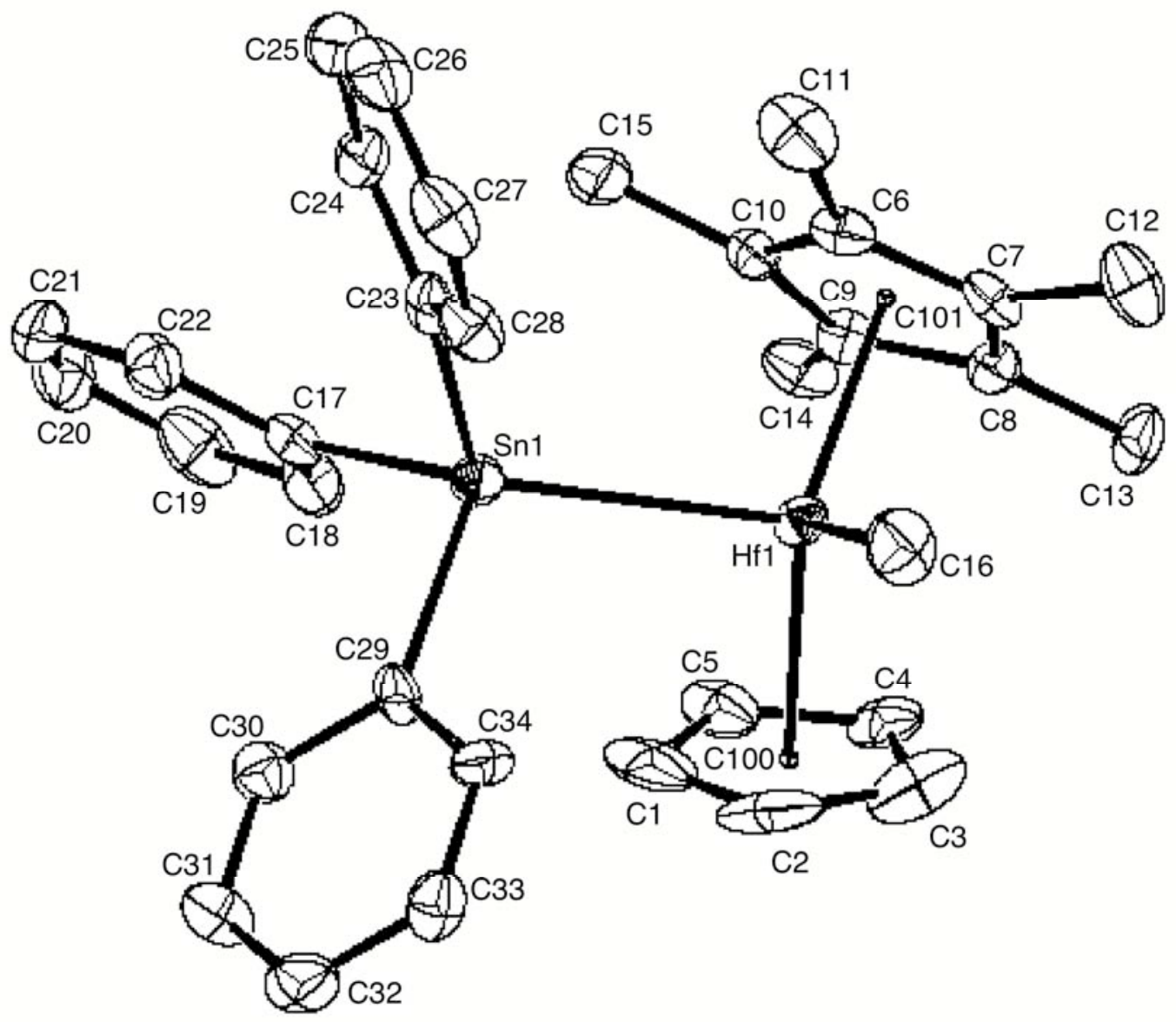


Figure 4. ORTEP diagram of $\mathrm{CpCp} * \mathrm{Hf}\left(\mathrm{SnPh}_{3}\right) \mathrm{OMe} \bullet 0.5\left(\mathrm{C}_{7} \mathrm{H}_{8}\right)\left(\mathbf{1 0} \cdot \mathbf{0 . 5}\left(\mathbf{C}_{7} \mathbf{H}_{\mathbf{8}}\right)\right)$. The hydrogen atoms and the $\mathrm{C}_{7} \mathrm{H}_{8}$ molecule were removed for clarity. Atoms are shown as $50 \%$ probability ellipsoids.

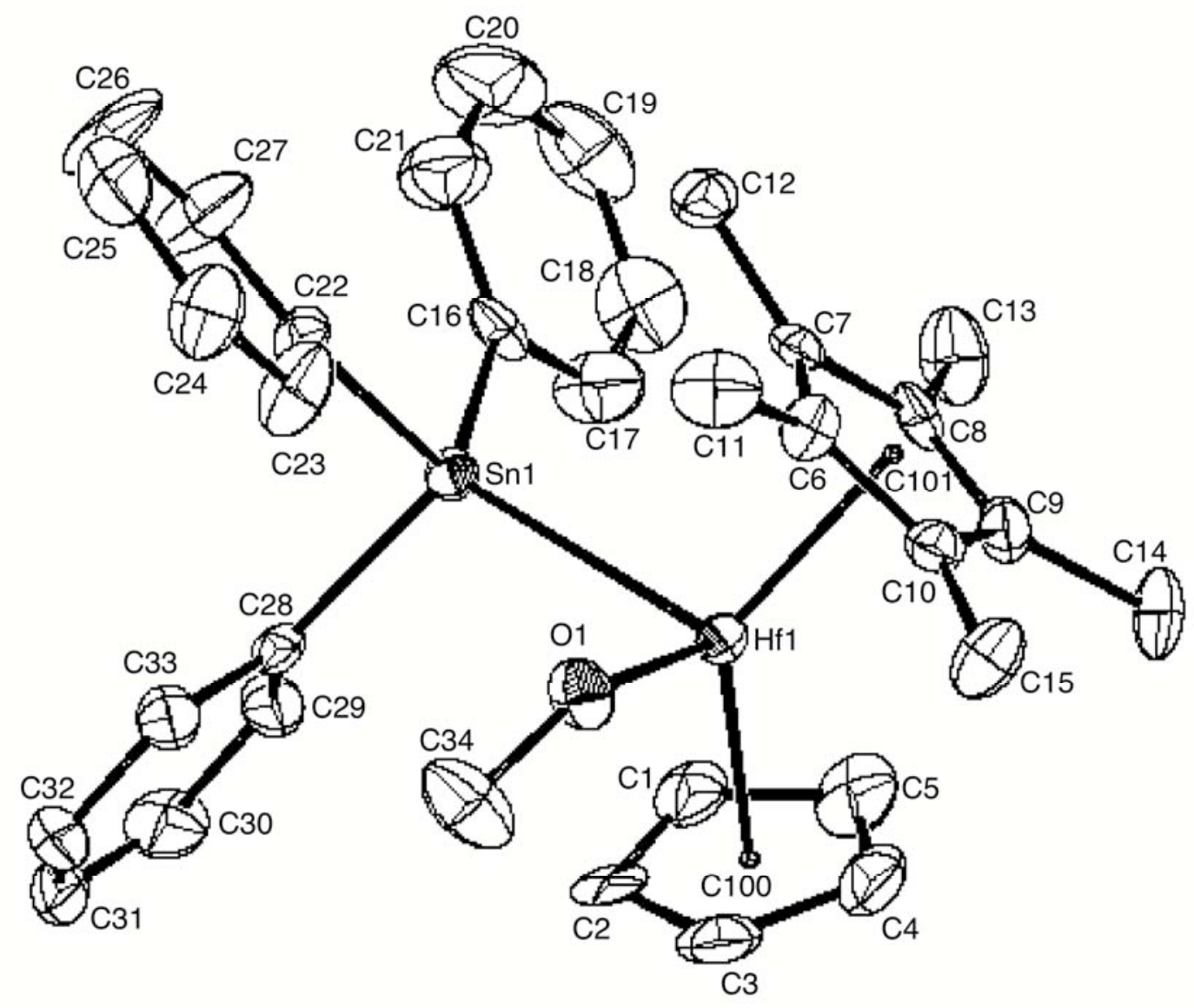


Figure 5. A representative first-order plot; $[9]_{\mathrm{i}}=14.5 \mathrm{mM}, \mathrm{T}=45^{\circ} \mathrm{C}$.

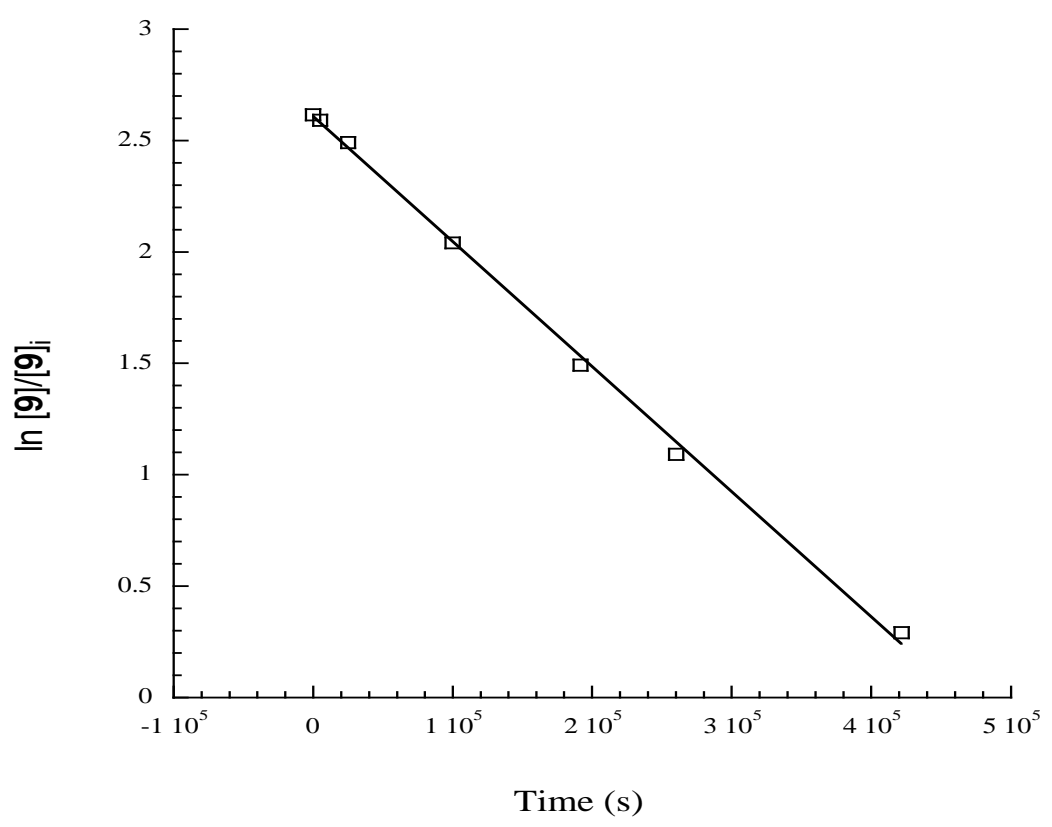

